\title{
Measurement of the azimuthal ordering of charged hadrons with the ATLAS detector
}

\author{
G. Aad et al.* \\ (ATLAS Collaboration)
}

(Received 2 March 2012; published 14 September 2012)

\begin{abstract}
This paper presents a study of the possible ordering of charged hadrons in the azimuthal angle relative to the beam axis in high-energy proton-proton collisions at the Large Hadron Collider (LHC). A spectral analysis of correlations between longitudinal and transverse components of the momentum of the charged hadrons, driven by the search for phenomena related to the structure of the QCD field, is performed. Data were recorded with the ATLAS detector at center-of-mass energies of $\sqrt{s}=900 \mathrm{GeV}$ and $\sqrt{s}=7 \mathrm{TeV}$. The correlations measured in a kinematic region dominated by low- $p_{T}$ particles are not well described by conventional models of hadron production. The measured spectra show features consistent with the fragmentation of a QCD string represented by a helixlike ordered gluon chain.
\end{abstract}

DOI: 10.1103/PhysRevD.86.052005

PACS numbers: $13.85 . \mathrm{Hd}, 13.87 . \mathrm{Fh}$

\section{INTRODUCTION}

Inclusive charged-particle distributions have been measured in proton-proton $(p p)$ collisions at the LHC for different center-of-mass energies [1-7]. These measurements provide insight into the strong interaction (QCD) at low energy scales and show that predictions of current phenomenological models cannot fully describe the measured observables in all kinematic regions. As shown in Ref. [2], the limitation of models is particularly apparent when particles with low transverse momentum $\left(p_{T}<500 \mathrm{MeV}\right)$ are studied. Specifically, discrepancies are observed in the description of

(i) the charged-particle density as a function of pseudorapidity, $d N_{\mathrm{ch}} / d \eta$

(ii) the shape of the charged-particle multiplicity distribution both at low and high multiplicities;

(iii) the shape of the charged-particle multiplicity distribution for both $p_{T}<500 \mathrm{MeV}$ and $p_{T}>3 \mathrm{GeV}$; and

(iv) correlations between the average $p_{T}$ of charged particles per event, $\left\langle p_{T}\right\rangle$, and the charged-particle multiplicity $n_{\mathrm{ch}}$.

Some of these discrepancies may be reduced through the development of parametrizations for the models of nonperturbative QCD and their adjustments (tunes) that better match model predictions to the latest measurements with particles produced at very low $p_{T}$. Nevertheless, it is also possible that a new formulation of certain components of these phenomenological models is needed.

Many of the difficulties in accurately describing observables dominated by low- $p_{T}$ QCD phenomena stem from the fact that there is often a combination of nonperturbative

*Full author list given at the end of the article.

Published by the American Physical Society under the terms of the Creative Commons Attribution 3.0 License. Further distribution of this work must maintain attribution to the author(s) and the published article's title, journal citation, and DOI. effects, including soft diffraction, low- $p_{T}$ parton scattering and hadronization. These effects act simultaneously in a given kinematic region and are difficult to separate experimentally. The recent ATLAS study of inclusive charged-particle production [2] shows that the sensitivity of measurements to some of these effects depends on the definition of the kinematic region in which the measurements are made. Typically, the more inclusively the sample is defined, or the more soft particle activity is added to the measurement, the larger the disagreement is between the data and the models.

Hadronization, the mechanism of hadron formation from quark and gluon cascades, must be taken into account in all measurements involving hadrons. The flows of energy, momentum and flavor of hadrons approximately follow those of partons [8]. The mechanism of hadron formation, however, can only be described with the aid of phenomenological models. The two main models employed in multipurpose event generators are the string (Lund) fragmentation model [9] and the cluster model [10].

A proposed modification of the Lund string model [11] evokes the possibility of the formation of a helixlike structured gluon field at the end of the parton cascade. Such a structure corresponds to the optimal packing of soft gluons in the phase space under helicity conservation requirements. Most notably, the helix string model imposes correlations between the adjacent break-up points along a string with observable effects in the inclusive $p_{T}$ distribution and in the azimuthal ordering of direct hadrons, i.e. hadrons produced directly from the string fragmentation.

In this paper, the correlations in the charged-hadron azimuthal angle $\phi$ [12] are studied for two different schemes of hadron ordering using data recorded with the ATLAS detector. The results are corrected for detector effects and compared with the predictions of various Monte Carlo (MC) event generators. The paper is organized as follows: the observables are described in Sec. II. Section III describes the ATLAS detector, and Sec. IV presents the MC samples used in the study. The track and event selection are detailed in 
Sec. V. The analysis and the uncorrected data distributions measured at the center-of-mass energy of $\sqrt{s}=7 \mathrm{TeV}$ are presented in Sec. VI. The procedure to correct for detector and selection effects and an overview of systematic uncertainties can be found in Sec. VII (additional information is provided in Appendix B). The last section of the paper is devoted to a comparison of corrected data with models and a discussion of the results. Appendix A presents results with $\sqrt{s}=900 \mathrm{GeV}$.

\section{OBSERVABLE QUANTITY}

The presence of azimuthal ordering, stemming from the underlying QCD string structure, is studied for two different variants of the helixlike ordered gluon field. As suggested in [11], the azimuthal ordering of hadrons should be observable with the help of a power spectrum defined according to the expected structure of the helix field. Assuming the break-up of the string occurs via tunneling [9], with the partons emerging at rest, the azimuthal direction of the hadron's intrinsic transverse momentum coincides with the phase of the helix string in the center of the string piece which forms the hadron. Hence, the azimuthal opening angle of two direct hadrons measures the phase difference between two corresponding points along the string, with the transverse plane defined with respect to the string axis. The analysis takes advantage of the fact that in soft $p p$ interactions, the QCD strings tend to be aligned along the beam axis.

In close analogy with [11], where the authors assume the helix winding is proportional to the rapidity difference between hadrons, we define the power spectrum

$$
S_{\eta}(\xi)=\frac{1}{N_{\mathrm{ev}}} \sum_{\text {event }} \frac{1}{n_{\mathrm{ch}}}\left|\sum_{j}^{n_{\mathrm{ch}}} \exp \left(i\left(\xi \eta_{j}-\phi_{j}\right)\right)\right|^{2},
$$

where $\xi$ is a parameter and $\eta_{j}\left(\phi_{j}\right)$ is the pseudorapidity (azimuthal angle) of the $j$-th hadron, $N_{\mathrm{ev}}$ is the number of events, and $n_{\mathrm{ch}}$ is the number of charged hadrons in the event. The inner sum runs over charged hadrons in the event and the outer sum over events in the sample.

It is important to note that the form of the helix field is not well constrained and that it is possible to find several parametrizations of the helix field conforming to the assumptions made in [11]. One possible scenario [13] corresponds to a static, regular helix structure with the phase difference $\Delta \phi$ proportional to the amount of internal energy stored in the fraction $f$ of string with mass $M_{0}$

$$
\Delta \phi=\mathcal{S} f M_{0}=\mathcal{S}_{\kappa} \Delta l=\mathcal{S} \Delta E,
$$

where $\mathcal{S}$ is a parameter, $\kappa$ is the string energy density, $\Delta \phi$ is the difference in the helix phase between two points along the string and $\Delta l$ and $\Delta E$ are the length and the energy, respectively, of the corresponding string piece in its rest frame. The energy-distance $\Delta E$ along the string between direct hadrons is not directly observable but according to MC studies, the signature of the helix-shaped field should be visible with help of a very loose approximation of the string by a chain of hadrons ordered in pseudorapidity. For the purpose of measuring the azimuthal ordering, we thus retain two parameters for each final hadron: the azimuthal angle $\phi$ and the position $X$ along the chain, evaluated as

$$
X_{j}=0.5 E_{j}+\sum_{k=0}^{k<j} E_{k}
$$

where $E_{k}$ is the energy of the $k$-th hadron in the chain, and the position of the hadron is associated with the center of the corresponding string piece. Accordingly, we define an alternative power spectrum

$$
S_{E}(\omega)=\frac{1}{N_{\mathrm{ev}}} \sum_{\text {event }} \frac{1}{n_{\mathrm{ch}}}\left|\sum_{j}^{n_{\mathrm{ch}}} \exp \left(i\left(\omega X_{j}-\phi_{j}\right)\right)\right|^{2},
$$

where $\omega$ is a parameter. The inner sum runs over pseudorapidity-ordered charged hadrons in the event.

The presence of a helixlike angular ordering of hadrons of either type would manifest itself as a peak in the corresponding power spectrum; the position of the peak would indicate the density of the helix winding. It should be stressed that, though formally very similar, $S_{E}$ and $S_{\eta}$ are only loosely correlated. A modified form of the helix string implies a difference in the experimental signature, such that the presence of a helix gluon field creating a peak in $S_{E}$ does not necessarily result in a peak structure in $S_{\eta}$ and vice-versa.

The power spectra can also be expressed as a sum of contributions from pairs of hadrons

$$
\begin{aligned}
& S_{\eta}(\xi)=1+\frac{1}{N_{\text {ev }}} \sum_{\text {event }} \frac{1}{n_{\mathrm{ch}}} \sum_{i \neq j} \cos \left(\xi \Delta \eta_{i j}-\Delta \phi_{i j}\right), \\
& S_{E}(\omega)=1+\frac{1}{N_{\mathrm{ev}}} \sum_{\text {event }} \frac{1}{n_{\mathrm{ch}}} \sum_{i \neq j} \cos \left(\omega \Delta X_{i j}-\Delta \phi_{i j}\right),
\end{aligned}
$$

where $\Delta \phi_{i j}=\phi_{i}-\phi_{j}$ is the opening azimuthal angle between hadrons, $\Delta \eta_{i j}=\eta_{i}-\eta_{j}$ is their pseudorapidity difference and $\Delta X_{i j}=X_{i}-X_{j}$ their energy-distance as defined above. The absence of correlations corresponds to $S_{\eta}(\xi)=1$ and $S_{E}(\omega)=1$.

\section{ATLAS DETECTOR}

The ATLAS detector [14] covers almost the entire solid angle around the collision point with layers of tracking detectors, calorimeters and muon chambers. It has been designed to study a wide range of physics topics at LHC energies. For the measurements presented in this paper, the trigger system and the tracking devices are of particular importance.

The ATLAS inner detector has full coverage in $\phi$ and covers the pseudorapidity range $|\eta|<2$.5. It consists of a 
silicon pixel detector, a silicon strip detector (SCT) and a transition radiation tracker (TRT). These detectors are immersed in a $2 \mathrm{~T}$ axial magnetic field. The pixel, SCT and TRT detectors have typical $r-\phi$ position resolutions of 10, 17 and $130 \mu \mathrm{m}$, respectively, and the pixel and SCT detectors have $r-z$ position resolutions of 115 and $580 \mu \mathrm{m}$, respectively. A track traversing the full radial extent would typically have 3 silicon pixel hits, 8 and more silicon strip hits and more than 30 TRT hits.

The ATLAS detector has a three-level trigger system: level 1 (L1), level 2 (L2) and the event filter (EF). For this measurement, the L1 trigger relies on the beam pickup timing devices (BPTX) and the minimum bias trigger scintillators (MBTS). The BPTX are composed of electrostatic beam pickups attached to the beam pipe at a distance $z= \pm 175 \mathrm{~m}$ from the center of the ATLAS detector. The MBTS are mounted at each end of the detector in front of the liquid-argon endcap-calorimeter cryostats at $z= \pm 3.56 \mathrm{~m}$ and are segmented into eight sectors in azimuth and two rings in pseudorapidity $(2.09<|\eta|<2.82$ and $2.82<|\eta|<3.84)$. Data were taken for this analysis using the single-arm MBTS trigger, formed from BPTX and MBTS L1 trigger signals. The MBTS trigger was configured to require one hit above threshold from either side of the detector. The MBTS trigger efficiency was studied with a separate prescaled L1 BPTX trigger, filtered to obtain inelastic interactions by inner detector requirements at L2 and EF [2].

\section{MONTE CARLO SAMPLES}

The analysis has been developed using a PYTHIA 6.421 [15] (MC09 tune [16]) sample with large statistics, fully simulated [17] and reconstructed using the standard ATLAS reconstruction chain [18]. It should be stressed that the choice of the MC tune does not affect the outcome of the analysis because the data are corrected in a modelindependent way.

For the comparison of the corrected data with the standard hadronization models, MC samples produced with PHOJET 1.12.1.35 [19], HERWIG + + 2.5.1 [20] (LHC-UE7-2/MU900-2 tunes [21]), PYTHIA 8.130 (4C tune [22]) and a recent tune of PYTHIA 6 (AMBT2b [23]) have been used. The MC generators PYTHIA and PHOJET employ the Lund string fragmentation model whereas the HERWIG MC is based on the cluster model. To study the sensitivity of the power spectra to the modification of the string fragmentation model, the data are also compared with an alternative implementation of the fragmentation process based on the helix string field described by Eq. (2) [24].

\section{DATA SAMPLES}

The measurements reported in this paper were made using $p p$ collision data recorded at $\sqrt{s}=7 \mathrm{TeV}$. The data were collected with stable colliding beams at $7 \mathrm{TeV}$ and correspond to an integrated luminosity of $\sim 190 \mu \mathrm{b}^{-1}$ from the beginning of the 2010 LHC run [2]. A sample of $p p$ collision events recorded at $\sqrt{s}=900 \mathrm{GeV}$ corresponding to an integrated luminosity of $\sim 7 \mu \mathrm{b}^{-1}$ [2] was also studied and the results are shown in Appendix A.

\section{A. Event and track selection}

Events are selected using the following criteria:

(i) the event has at least one trigger hit in MBTS;

(ii) the event has one and only one reconstructed vertex and this vertex must have at least three associated tracks;

(iii) the event has no tracks with $p_{T}>10 \mathrm{GeV}$;

(iv) the event has at least six reconstructed tracks $\left(n_{\mathrm{tr}}>5\right)$ passing the requirements below.

The requirements on reconstructed tracks included in the analysis are the following:

(i) the track is reconstructed by the track reconstruction algorithm used in [1], with an implicit cut on the transverse momentum, $p_{T}>100 \mathrm{MeV}$, and more than 6 hits in the silicon detectors;

(ii) the track has a transverse impact parameter with respect to the primary vertex $\left|d_{0}^{\mathrm{PV}}\right|<2 \mathrm{~mm}$;

(iii) the track has a longitudinal impact parameter with respect to the primary vertex $\left|z_{0}^{\mathrm{PV}}\right| \sin (\theta)<2 \mathrm{~mm}$; and

(iv) the track is reconstructed in the pseudorapidity range $-2.5<\eta<2.5$.

The requirement on the minimum number of tracks ensures full trigger efficiency [2]. The contributions from the beam and noncollision background (cosmic rays and detector noise) have been investigated in [2] and found to be negligible. Events with multiple primary vertices (less than $0.3 \%$ of the sample and subsamples defined below) are rejected in order to prevent a bias from multiple $p p$ interactions in the colliding proton bunches.

\section{B. Subsample definitions}

The analysis is carried out in parallel on the sample selected as described above (henceforth referred to as the "inclusive sample") and on two subsamples.

The first subsample contains events where the transverse momentum of any reconstructed track does not exceed $1 \mathrm{GeV}\left(\max \left(p_{T}\right)<1 \mathrm{GeV}\right)$. This subsample is called the "low- $p_{T}$ enhanced sample." The effects of parton showering and lateral boost are diminished in this selection. The $\max \left(p_{T}\right)<1 \mathrm{GeV}$ requirement selects events with little acollinear jet activity and thus the transverse activity is expected to be primarily sensitive to hadronization effects.

The analysis is also performed on a second subsample defined by a higher track $p_{T}$ cutoff, $p_{T}>500 \mathrm{MeV}$. This particular selection yields a subsample with a significantly reduced contribution from diffractive $p p$ interactions 
TABLE I. Average charged-particle multiplicity $N_{\mathrm{ch}}^{\mathrm{gen}}$ and relative fraction of diffractive events for the fully simulated PYTHIA6 (MC09) MC sample at $\sqrt{s}=7 \mathrm{TeV}$. Results are shown for events selected with the corrected charged-track multiplicity cutoff $n_{\mathrm{ch}}>10$ (detector level) and $n_{\mathrm{ch}}^{\mathrm{gen}}>10$ (particle level) (* indicates before the $\max \left(p_{T}\right)$ cut correction).

\begin{tabular}{lccc}
\hline \hline Model (tune) & \multicolumn{3}{c}{ PYTHIA6(MC09) } \\
\hline low $p_{T}$ cut & \multicolumn{2}{c}{$>100 \mathrm{MeV}$} & $>500 \mathrm{MeV}$ \\
$\max \left(p_{T}\right)$ cut & $<10 \mathrm{GeV}$ & $<1 \mathrm{GeV}$ & $<10 \mathrm{GeV}$ \\
& $n_{\mathrm{ch}}>10$ & (corrected detector-level) \\
$N_{\text {ch }}^{\text {gen }}$ & 31.93 & $17.11^{*}$ & 22.62 \\
diffractive/total & $3.9 \pm 0.1 \%$ & $21.4 \pm 0.2 \%$ & $<0.1 \%$ \\
\multicolumn{4}{c}{$n_{\mathrm{ch}}^{\text {gen }}>10$ (particle-level) } \\
$N_{\text {ch }}^{\text {gen }}$ & 31.31 & 15.53 & 22.26 \\
diffractive/total & $4.0 \pm 0.1 \%$ & $22.7 \pm 0.2 \%$ & $<0.1 \%$ \\
\hline \hline
\end{tabular}

(see Table I). This subsample is referred to as the "low- $p_{T}$ depleted sample".

\section{Selection criteria at particle level}

The comparison between corrected data and MC models requires an adjustment of the event selection in order to avoid a systematic bias. The analysis relies on two main selection criteria: the charged-hadron $p_{T}$ and the chargedhadron multiplicity.

The effect of the low $p_{T}$ cutoff is easily modeled at the particle level (MC truth [25]) and the corresponding systematic uncertainty is covered by the uncertainty assigned to the correction procedure, described in Sec. VII. The cut on the maximal $p_{T}$ of a track is more selective when applied at the particle level as it removes also those MC events which contain a nonreconstructed high $p_{T}$ track. The effect is non-negligible in the low- $p_{T}$ enhanced sample, where it is corrected for and a systematic uncertainty is assigned to reflect the additional uncertainty.

The charged-track multiplicity selection criteria on the measured data need to be modified in order to emulate MC modelling. The adjustment of the charged-track multiplicity is done in the following way: for each reconstructed track, a random number RND is repeatedly generated according to a flat distribution until RND $<\epsilon_{\text {tr }}, \epsilon_{\text {tr }}$ being the estimated track reconstruction efficiency [26]. The corrected charged-track multiplicity $n_{\mathrm{ch}}$ corresponds to the number of random numbers generated for the entire event. The procedure also contains an additional correction for the residual content of secondary tracks.

The selection based on $n_{\mathrm{ch}}$ roughly reproduces the average charged-particle multiplicity of the particle-level sample selected with a $n_{\mathrm{ch}}^{\mathrm{gen}}$ cutoff (see Table I). Figure 1 illustrates the effect of hadron-level cuts on the true charged-particle multiplicity $n_{\mathrm{ch}}^{\mathrm{gen}}$. The choice of the selection cut for the current analysis $\left(n_{\mathrm{ch}} \approx n_{\mathrm{ch}}^{\mathrm{gen}}>10\right)$ is aimed

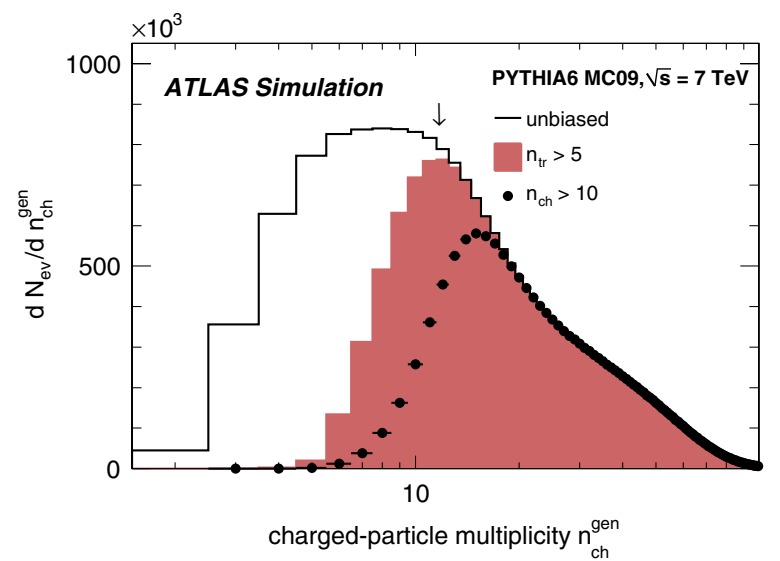

FIG. 1 (color online). The impact of the charged-track multiplicity cutoff on the true charged-particle multiplicity distribution. The arrow indicates the cut on the true charged-particle multiplicity, removing the region affected by the loss of events due to the requirement of at least six reconstructed tracks (white area). The shaded area corresponds to the detector-level cut $n_{\mathrm{tr}}>5$. The final analysis selection cut $\left(n_{\mathrm{ch}}>10\right)$ is indicated by closed points.

at minimizing any bias in the power spectra related to the loss of events due to the detector-level charged-track multiplicity cutoff $n_{\mathrm{tr}}>5$.

The selection criteria distinguishing between data samples are summarized in Table II, which also provides the information about the final number of events retained for the analysis and the mean corrected charged-track multiplicity of each sample. The relative fraction of diffractive events (based on the nominal cross-section) and the mean charged-particle multiplicity of the Monte-Carlo selection are given in Table I.

The final track selection contains $2.8 \pm 0.4 \%$ of secondary tracks according to studies performed on simulated samples. Nonprimary tracks predominantly arise from hadronic interactions with detector material, photon conversion to electron-positron pairs and decays of long-lived particles. The average reconstruction efficiency for primary charged particles is $75 \%$ for the $p_{T}>100 \mathrm{MeV}$ track selection and $84 \%$ for the $p_{T}>500 \mathrm{MeV}$ selection. The systematic uncertainty due to the performance of the track reconstruction is estimated following the studies performed in Ref. [2].

TABLE II. Number of selected data events and average corrected charged-track multiplicity, per sample (* indicates before the $\max \left(p_{T}\right)$ cut correction).

\begin{tabular}{lccccc}
\hline \hline \multicolumn{4}{c}{$p p$ collisions at $\sqrt{s}=7 \mathrm{TeV}}$, & $n_{\mathrm{ch}}>10$ \\
\hline \multicolumn{4}{c}{$p_{T}>100 \mathrm{MeV}$} & $p_{T}>500 \mathrm{MeV}$ \\
$\max \left(p_{T}\right)<10 \mathrm{GeV}$ & $\max \left(p_{T}\right)<1 \mathrm{GeV}$ & $\max \left(p_{T}\right)<10 \mathrm{GeV}$ \\
$N_{\mathrm{ev}}$ & $N_{\mathrm{ch}}$ & $N_{\mathrm{ev}}$ & $N_{\mathrm{ch}}$ & $N_{\mathrm{ev}}$ & $N_{\mathrm{ch}}$ \\
8099211 & 34.71 & 1292389 & $17.96^{*}$ & 4341217 & 23.27 \\
\hline \hline
\end{tabular}




\section{ANALYSIS METHOD}

For the measurement of $S_{E}$, the selected tracks are ordered by pseudorapidity and a pion mass is assigned to each of them. According to MC estimates, the chargedparticle sample contains about $86 \%$ pions, $9.5 \%$ kaons, $4 \%$ protons/antiprotons and a negligible number of leptons $(\sim 0.5 \%)$. The effect of assuming a pion mass would need to be taken into account for a precision measurement of the position of the signal but its impact on the comparison of data with MC models is negligible. For the calculation of $S_{\eta}$ no mass assumption is required. The power spectra are measured as $\left(S_{\eta}-1\right)$ and $\left(S_{E}-1\right)$ for convenience.

The uncorrected power spectra are shown in Fig. 2 as a function of the azimuthal opening angle (helix phase difference) per unit of energy distance $\left(S_{E}\right)$ and per unit of pseudorapidity $\left(S_{\eta}\right)$ for the three samples. All angles are expressed in radians throughout the paper.

MC studies show that the power spectra are sensitive to various kinds of correlations between particles. The dominant peaks seen in both distributions arise due to the jet structure and momentum conservation in the hard partonparton scattering. The position of these peaks depends on the
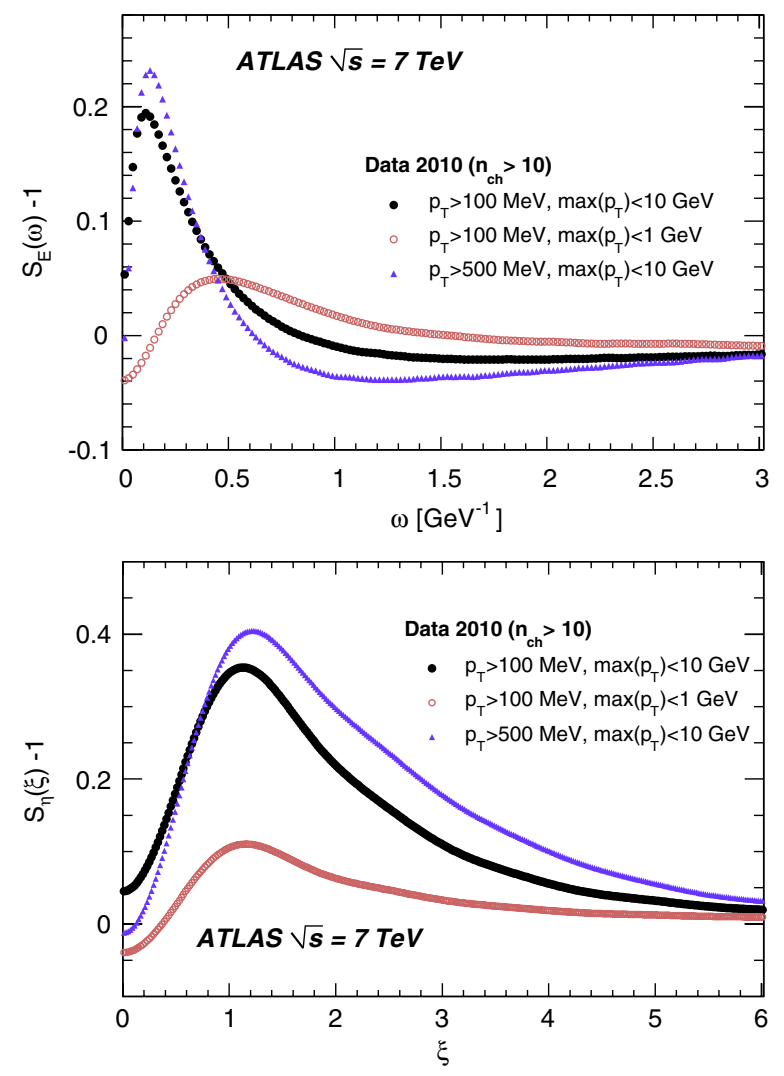

FIG. 2 (color online). The uncorrected data measurements (top: $S_{E}$, bottom: $S_{\eta}$ ) obtained from the data sample collected at $\sqrt{s}=7 \mathrm{TeV}$. The measurement of the inclusive sample is compared to measurements of the low- $p_{T}$ enhanced and low- $p_{T}$ depleted subsamples. visible energy (in the case of $S_{E}$ ) and the pseudorapidity range (in the case of $S_{\eta}$ ) used in the analysis. Their height is sensitive to a number of physics processes, notably the amount and structure of multiple parton interactions, cross-talk between overlapping hadronic systems (color reconnection), and parton shower properties. Note, that the values of $S_{E}(\omega=0)$ and $S_{\eta}(\xi=0)$ are identical by definition. They are closely related to the average opening angle between particles in the transverse plane. The presence of azimuthal correlations stemming from the properties of the gluon field should be visible as an additional positive peak or enhancement in the power spectrum and the modification should be more pronounced in the low- $p_{T}$ region, where the fragmentation and parton interactions have comparable effects on the transverse momentum of hadrons.

The comparison of the uncorrected data obtained in the inclusive event selection and in the low- $p_{T}$ enhanced/depleted subsamples shows the size of the peaks diminishing with the decreasing track $p_{T}$ selection range, a feature we may associate with the relative fraction of high $p_{T}$ jets in the sample.

\section{CORRECTION PROCEDURE AND SYSTEMATIC UNCERTAINTIES}

The data are corrected for nonreconstructed charged particles with the help of an unfolding technique based on [27] and described in Appendix B.

The correction for the secondary track content is obtained using a random sampling of secondary tracks according to the parametrized secondary track rate obtained from fully simulated MC. The contribution from tracks labeled as secondary ( $\delta S^{\mathrm{sec}}$ ) is subtracted from the measured data distribution. Typically, it amounts to $\sim 6 \%$ of the size of the peak in the power spectrum.

An additional correction is applied in the low- $p_{T}$ enhanced sample to compensate for the bias introduced by the selection cut on $\max \left(p_{T}\right)$ in case the track with highest $p_{T}$ was not reconstructed. Selecting events with exactly one track above the threshold, the power spectra $\left(S^{p_{T}}\right)$ are calculated using all the other tracks in the event. The correction is obtained by subtracting $S^{p_{T}}$ from the data in the proportion corresponding to the probability for the high- $p_{T}$ track being lost in the reconstruction.

TABLE III. The parametrization of the components of the systematic uncertainty, per measured point of the corrected power spectrum.

\begin{tabular}{lc}
\hline \hline Source & Systematic uncertainty $\left[S_{E}(\omega), S_{\eta}(\xi)\right]$ \\
\hline $\begin{array}{l}\text { folding procedure } \\
\text { unfolding }\end{array}$ & $\max (0.003,3 \%(S-1))$ \\
& envelope of the residual bias \\
tracking efficiency & from unfolding \\
secondary tracks & scaling parameters $\pm 5 \%$ \\
$n_{\mathrm{ch}}$ cutoff & $\max \left(0.005,0.25\left|\delta S^{\mathrm{sec}}\right|\right)$ \\
$\max \left(p_{T}\right)<1 \mathrm{GeV}$ & variation of the cutoff $n_{\mathrm{ch}}$ by \pm 1 \\
\hline \hline
\end{tabular}


All corrections are model independent. The correction procedure has been verified by checking the procedure on fully simulated MC samples.

\section{A. Systematic uncertainties}

The principal sources and parametrizations of systematic uncertainty associated with the corrected data are summarized in Table III. The combined systematic uncertainty has the following components:

(i) residual bias of the folding procedure (Appendix B): obtained from the comparison of distributions reconstructed at the detector level using samples with full detector simulation and those obtained with the folding technique;

(ii) uncertainty of the unfolding technique: parametrized to cover the residual discrepancies in the scaling of 3 folding iterations (Appendix B);

(iii) uncertainty on the tracking efficiency estimate: dominated by the uncertainty on the inner detector material description, which translates into a variation of scaling factors by $5 \%$;
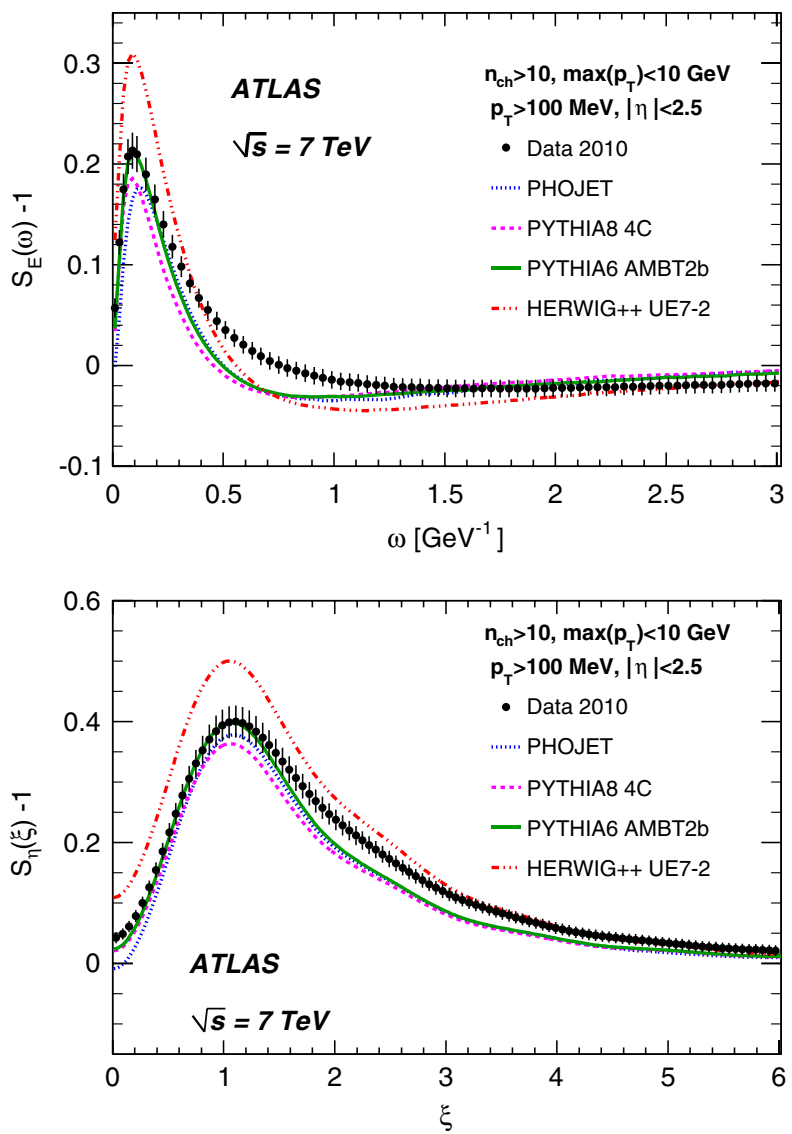

FIG. 3 (color online). Corrected data from the inclusive sample compared to particle-level predictions from various $\mathrm{MC}$ models using conventional hadronization algorithms. The top and bottom plots are for the $S_{E}$ and $S_{\eta}$ power spectra, respectively. The error bars correspond to the combined statistical and systematic uncertainties. (iv) uncertainty due to the residual content of secondary tracks: set to $25 \%$ of the correction applied, with minimal value of 0.005 (based on MC studies);

(v) uncertainty due to the difference in the chargedparticle multiplicity selection at the generator level and at the detector level: calculated in a modelindependent way as a variation of the shape corresponding to the change of the averaged selected charged-particle multiplicity by one unit; and

(vi) the uncertainty in the correction of the bias due the $\max \left(p_{T}\right)$ cut: corresponds to a $5 \%$ variation of the track reconstruction efficiency.

All contributions to the systematic uncertainty are combined quadratically. The negative correlation between track reconstruction efficiency and secondary track content is neglected, making the uncertainty estimate more conservative.

\section{RESULTS}

The results of this analysis obtained for $p p$ collisions at $\sqrt{s}=7 \mathrm{TeV}$ are presented in this section. Results from
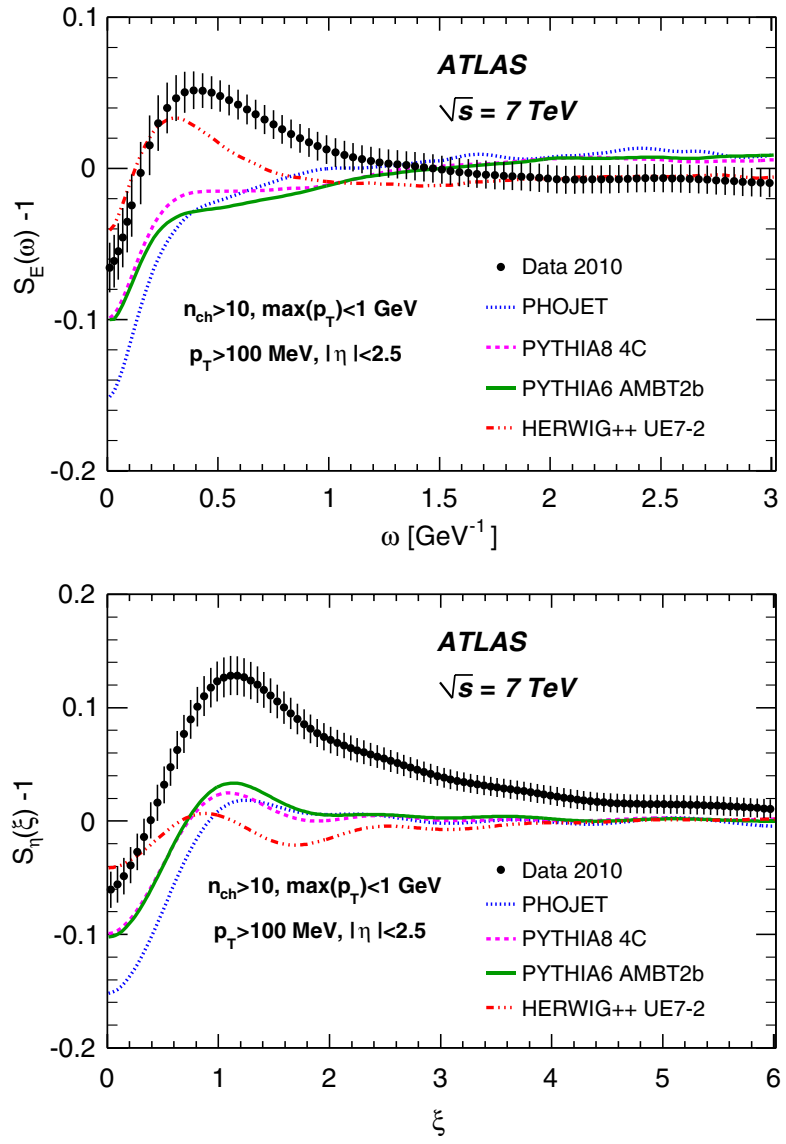

FIG. 4 (color online). Corrected data from the low- $p_{T}$ enhanced sample compared to particle-level predictions from various MC models using conventional hadronization algorithms. The top and bottom plots are for the $S_{E}$ and $S_{\eta}$ power spectra, respectively. The error bars correspond to the combined statistical and systematic uncertainties. 
this analysis repeated for $\sqrt{s}=900 \mathrm{GeV}$ are shown in Appendix A. The corrected data are compared with the predictions of several commonly used MC models: PYTHIA6, PHOJET, PYTHIA8 and HERWIG ++ .

Figure 3 shows the comparison for the inclusive event selection $\left(n_{\mathrm{ch}}>10, p_{T}>100 \mathrm{MeV}\right.$ and maximal $p_{T}<10 \mathrm{GeV}$ ). The principal peak structure observed in the power spectra for both $S_{E}$ and $S_{\eta}$ is roughly reproduced by PYTHIA and PHOJET models and overestimated by HERWIG ++ . The tail of the $S_{E}$ distribution around $0.5<\omega<1 \mathrm{rad} / \mathrm{GeV}$ is not reproduced by any of the models.

Hadronization effects should become more evident when measurements are made in regions of the phase space dominated by the production of low- $p_{T}$ particles. Figure 4 shows the power spectra measured in the low- $p_{T}$ enhanced sample $\left(n_{\mathrm{ch}}>10, p_{T}>100 \mathrm{MeV}\right.$ and maximal $\left.p_{T}<1 \mathrm{GeV}\right)$. A significant amount of correlations in observed in the data in both $S_{E}$ and $S_{\eta}$ distributions compared to the PHOJET and PYTHIA based models. HERWIG ++ gives a seemingly better description for the $S_{E}$ distribution yet it seems its prediction is more of an artifact of an enhanced single jet
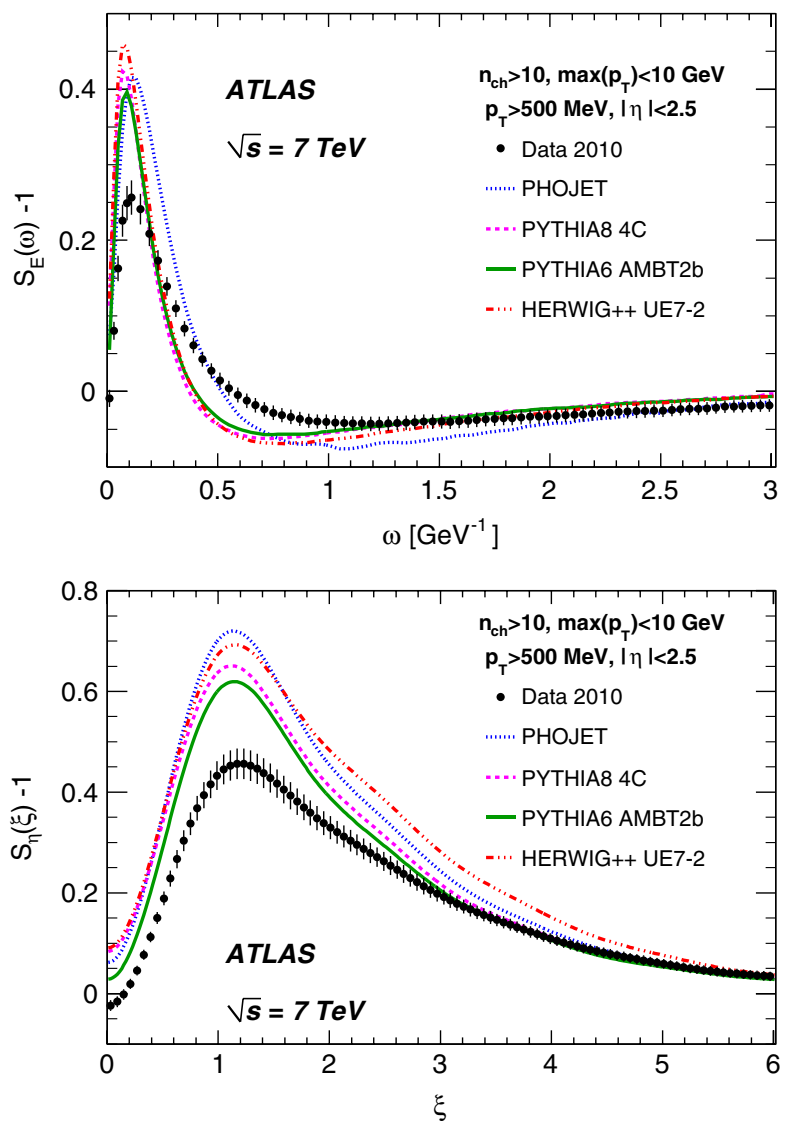

FIG. 5 (color online). Corrected data from the low- $p_{T}$ depleted sample compared to particle-level predictions from various MC models using conventional hadronization algorithms. The top and bottom plots are for the $S_{E}$ and $S_{\eta}$ power spectra, respectively. The error bars correspond to the combined statistical and systematic uncertainties. structure given the fact the model overestimates the measurements in the inclusive event selection (Fig. 3). The interpretation of this measurement in terms of the azimuthal ordering of hadrons related to the properties of the gluon field is discussed in Sec. VIII A.

Figure 5 shows the power spectra $S_{E}$ and $S_{\eta}$ for the corrected data and $\mathrm{MC}$ predictions in the low- $p_{T}$ depleted region $\left(n_{\mathrm{ch}}>10, p_{T}>500 \mathrm{MeV}\right.$ and maximal $p_{T}<10 \mathrm{GeV}$ ). In principle, this should be the best understood part of the phase space, with a suppressed diffractive component, lower sensitivity to hadronization effects and best available model tunes. However, we find that all models significantly overestimate the size of the principal peak structure in both $S_{E}$ and $S_{\eta}$.

Comparison of Figs. 3-5 show that the azimuthal correlations are qualitatively different in each subsample, and that the standard MC models fail to reproduce these accurately. Similar conclusion can be drawn for the measurement performed at $\sqrt{s}=900 \mathrm{GeV}$ (Appendix A).

In the frame of the conventional QCD modelling, we have tried to identify the most likely source of the observed
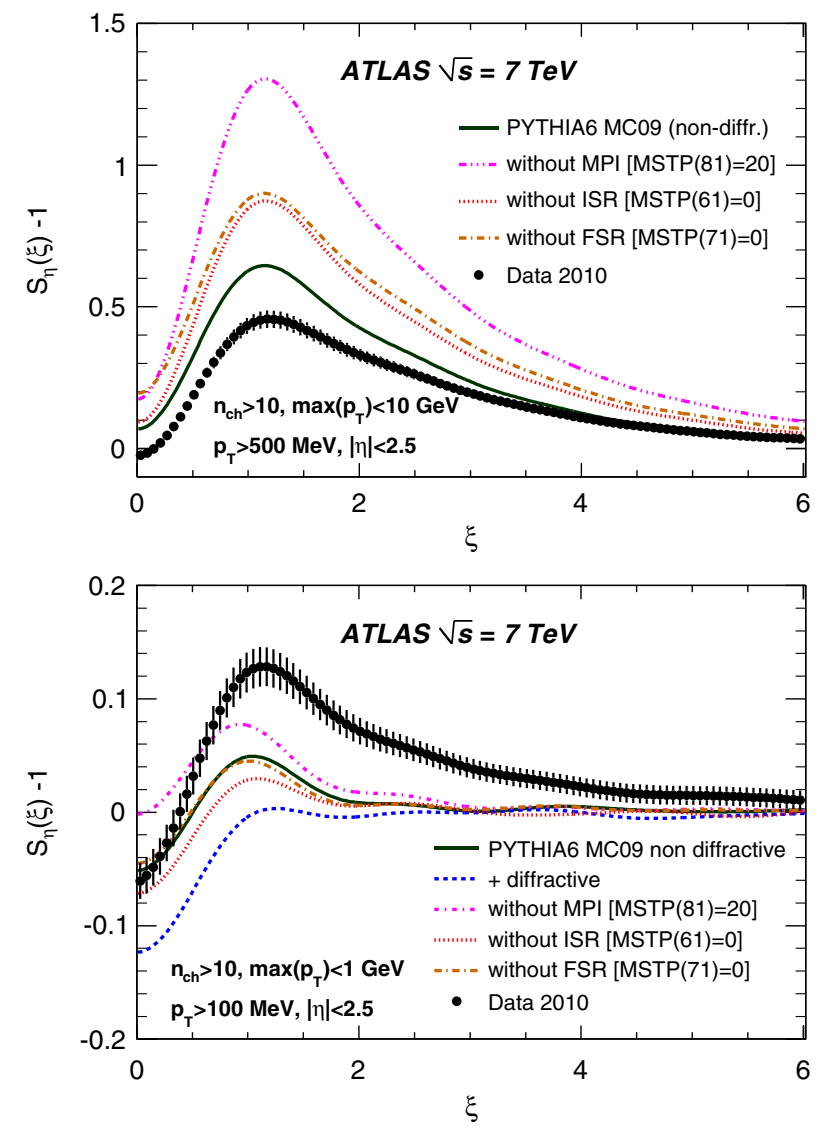

FIG. 6 (color online). Corrected $S_{\eta}$ distributions compared with the particle-level predictions of PYTHIA6 MC09 for various settings, using the nondiffractive $p p$ scattering (full line) as the baseline. Top: low- $p_{T}$ depleted subsample. Bottom: low- $p_{T}$ enhanced subsample. The error bars correspond to the combined statistical and systematic uncertainties. 
discrepancies. Figure 6 shows the sensitivity of the $S_{\eta}$ distribution to various components of the QCD modeling implemented in PYTHIA 6, taking as a baseline the nondiffractive $p p$ scattering scenario (indicated by the full line). In the low- $p_{T}$ depleted sample, the size of correlations varies strongly with the amount of multiple parton interactions (MPI), of initial state radiation (ISR) and with the amount of parton showering. The data prefer modelling with enhanced radiation and/or enhanced MPI rate which can be achieved via careful adjustment of the relevant model parameters.

However, such an adjustment typically creates an even larger discrepancy in the low- $p_{T}$ enhanced region, where the parton shower and ISR have smaller influence. Inversely, the removal of the MPI and of the diffractive processes increases the size of the peak in the modeling of the low- $p_{T}$ enhanced region, but none of these rather extreme variations lead to a good agreement with the measured $S_{\eta}$ distribution, while creating a huge discrepancy in the low- $p_{T}$ enhanced region. We conclude that for both measured spectra, the conventional models fail to describe the low- $p_{T}$ enhanced region, where the data are consistently showing a larger and broader peak structure.
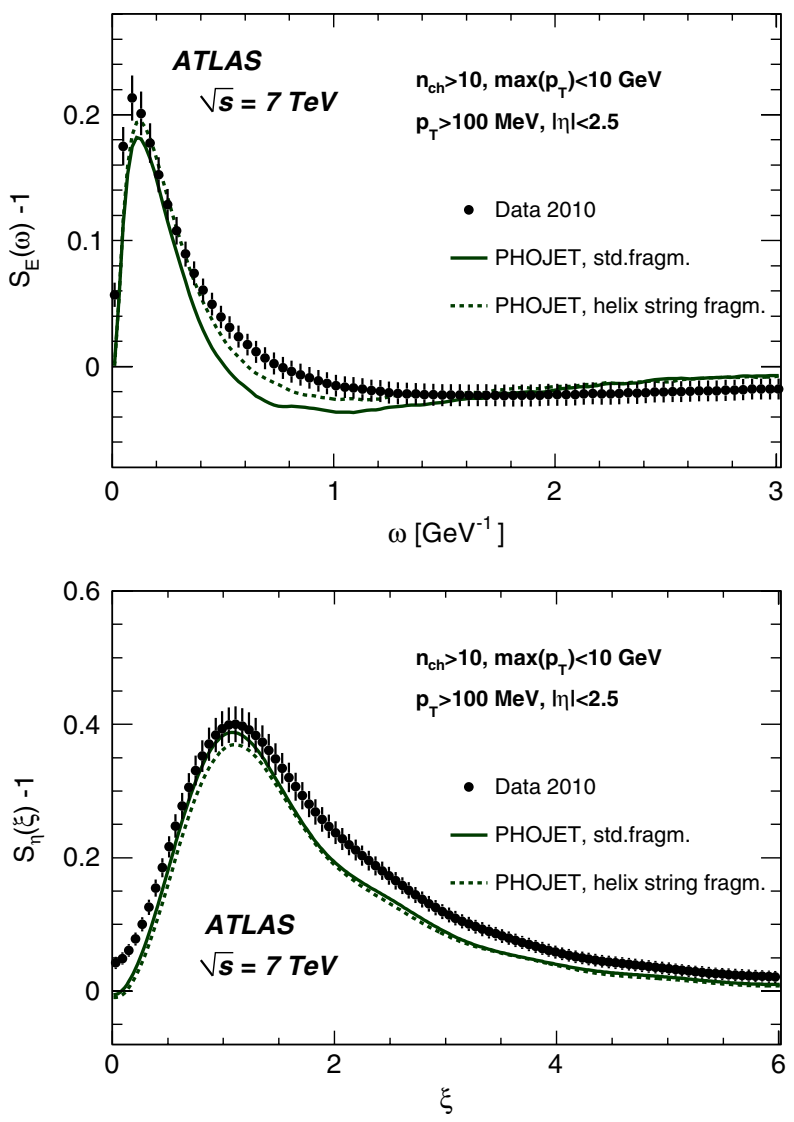

FIG. 7 (color online). Corrected $S_{E}$ (top) and $S_{\eta}$ (bottom) distributions compared with the particle-level predictions of PHOJET, interfaced to the PYTHIA6-based alternative fragmentation using helix string model [24] (setting $\mathcal{S}=0.7 \mathrm{rad} / \mathrm{GeV}$, see Eq. (2)), in the inclusive event selection. The error bars correspond to the combined statistical and systematic uncertainties.

\section{A. Alternative fragmentation model}

The question of compatibility of the measurements with the azimuthal ordering signal originating from the underlying structure of the QCD field is studied using the PYTHIA6-based helix string model implementation [24] of the modified helix string scenario corresponding to the $S_{E}$ definition (Eq. (2)). The comparison of corrected data with the PHOJET modelling of $p p$ interactions, interfaced alternatively with the standard string fragmentation and with the helix string fragmentation, is shown in Figs. 7 and 8. It is seen that the helixlike gluon ordering improves the description of the data in the inclusive sample for $S_{E}$ as it generates higher values in the region of $\omega \in$ $(0.5,1)$. In the low- $p_{T}$ enhanced sample the data are more strongly peaked than the model. This indicates the data have a more jetlike structure than predicted by PHOJET which means the model may need readjustment beyond the fragmentation part.

It is possible that the original helix string proposal [11] provides an improved description of the $S_{\eta}$ measurement but we cannot verify this hypothesis due to the absence of
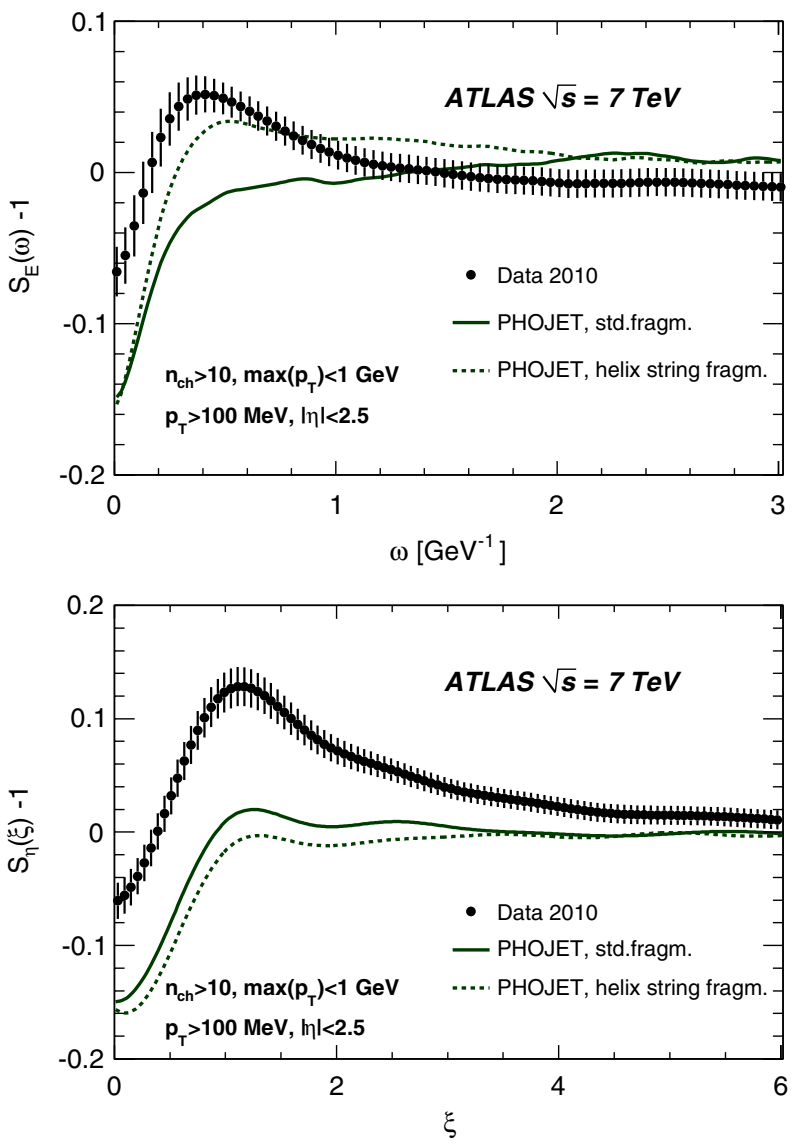

FIG. 8 (color online). Corrected $S_{E}$ (top) and $S_{\eta}$ (bottom) distributions compared with the particle-level predictions of PHOJET, interfaced to the PYTHIA6-based alternative fragmentation using helix string model [24] (setting $\mathcal{S}=0.7 \mathrm{rad} / \mathrm{GeV}$, see Eq. (2)), in the low- $p_{T}$ enhanced subsample. The error bars correspond to the combined statistical and systematic uncertainties. 
the MC implementation of this variant of the helix string model for parton configurations containing hard gluons.

In principle, the position of the azimuthal ordering signal allows a measurement of the characteristic density of the helix string winding (parameter $\mathcal{S}$ in Eq. (2)). The data indicate $\mathcal{S}$ in the range 0.5 to $0.9 \mathrm{rad} / \mathrm{GeV}$. However, the interplay between string fragmentation and the other processes (such as parton showering, color reconnection and multiple interactions) is not sufficiently understood to allow a more precise estimate (the measurement has to rely on MC description for the correction of the longitudinal boost).

\section{CONCLUSIONS}

A measurement of the ordering of charged hadrons in the azimuthal angle with the ATLAS data recorded from protonproton collisions at $\sqrt{s}=7 \mathrm{TeV}$ and $\sqrt{s}=900 \mathrm{GeV}$ has been presented.

A spectral analysis of correlations between the opening azimuthal angle and the longitudinal separation of the charged hadrons was performed by measuring the $S_{E}$ and $S_{\eta}$ power spectra. These measurements were done in three kinematic regions (inclusive, low- $p_{T}$ enhanced and low- $p_{T}$ depleted samples) that were specifically defined to help assess the potential contribution of hadronization effects to the power spectra by varying the levels of competition between hadronization and other QCD effects.

The results were compared with the expectations of various MC event generators. Predictions generated by the MC models employing the standard Lund string fragmentation model roughly reproduce the data in the inclusive sample.

The models systematically overestimate the size of correlations in the low- $p_{T}$ depleted sample, where the observables are sensitive to the multiple jet structure of events (due to the presence of underlying event and/or parton showering).

For observables measured in the low- $p_{T}$ enhanced sample, none of the models investigated describes the data adequately. A study showing the impact of extreme variations in MC model parameters that are known to contribute to soft-QCD effects demonstrates that, although some improvement of predictions for $S_{E}$ and $S_{\eta}$ can be achieved in the low- $p_{T}$ enhanced sample, it is still far from satisfactory.

The measurement of $S_{E}$ in the kinematic region dominated by low- $p_{T}$ particles shows features similar to those seen in models in which the fragmenting QCD strings are represented by helixlike ordered gluon chains. These measurements suggest that the inclusion of such azimuthally-ordered fragmentation effects could be one factor in improving current models of soft particle production and hadronization.

\section{ACKNOWLEDGMENTS}

We thank CERN for the very successful operation of the LHC, as well as the support staff from our institutions without whom ATLAS could not be operated efficiently. We acknowledge the support of ANPCyT, Argentina; YerPhI, Armenia; ARC, Australia; BMWF, Austria; ANAS, Azerbaijan; SSTC,
Belarus; CNPq and FAPESP, Brazil; NSERC, NRC and CFI, Canada; CERN; CONICYT, Chile; CAS, MOST and NSFC, China; COLCIENCIAS, Colombia; MSMT CR, MPO CR and VSC CR, Czech Republic; DNRF, DNSRC and Lundbeck Foundation, Denmark; ARTEMIS, European Union; IN2P3CNRS, CEA-DSM/IRFU, France; GNAS, Georgia; BMBF, DFG, HGF, MPG and AvH Foundation, Germany; GSRT, Greece; ISF, MINERVA, GIF, DIP and Benoziyo Center, Israel; INFN, Italy; MEXT and JSPS, Japan; CNRST, Morocco; FOM and NWO, Netherlands; RCN, Norway; MNiSW, Poland; GRICES and FCT, Portugal; MERYS (MECTS), Romania; MES of Russia and ROSATOM, Russian Federation; JINR; MSTD, Serbia; MSSR, Slovakia; ARRS and MVZT, Slovenia; DST/NRF, South Africa; MICINN, Spain; SRC and Wallenberg Foundation, Sweden; SER, SNSF and Cantons of Bern and Geneva, Switzerland; NSC, Taiwan; TAEK, Turkey; STFC, the Royal Society and Leverhulme Trust, United Kingdom; DOE and NSF, United States of America. The crucial computing support from all WLCG partners is acknowledged gratefully, in particular, from CERN and the ATLAS Tier-1 facilities at TRIUMF (Canada), NDGF (Denmark, Norway, Sweden), CC-IN2P3 (France), KIT/GridKA (Germany), INFN-CNAF (Italy), NL-T1 (Netherlands), PIC (Spain), ASGC (Taiwan), RAL (UK) and BNL (USA) and in the Tier-2 facilities worldwide.

\section{APPENDIX A}

The analysis is repeated for ATLAS data collected at $\sqrt{s}=900 \mathrm{GeV}$. The number of selected events and the

TABLE IV. Number of selected events and average corrected charged-track multiplicity, per sample (* indicates before the $\max \left(p_{T}\right)$ cut correction).

\begin{tabular}{lccccc}
\hline \hline \multicolumn{4}{c}{$p p$ collisions at $\sqrt{s}=900 \mathrm{GeV}}$, & $n_{\mathrm{ch}}>10$ \\
\hline \multicolumn{4}{c}{$p_{T}>100 \mathrm{MeV}$} & \multicolumn{2}{c}{$p_{T}>500 \mathrm{MeV}$} \\
$\max \left(p_{T}\right)<10 \mathrm{GeV}$ & $\max \left(p_{T}\right)<1 \mathrm{GeV}$ & $\max \left(p_{T}\right)<10 \mathrm{GeV}$ \\
$N_{\mathrm{ev}}$ & $N_{\mathrm{ch}}$ & $N_{\mathrm{ev}}$ & $N_{\mathrm{ch}}$ & $N_{\mathrm{ev}}$ & $N_{\mathrm{ch}}$ \\
224717 & 24.8 & 59880 & $17.7^{*}$ & 68456 & 16.3 \\
\hline \hline
\end{tabular}

TABLE V. Average charged-particle multiplicity $N_{\mathrm{ch}}^{\mathrm{gen}}$ and relative fraction of diffractive events for the fully simulated PYTHIA6 (MC09) MC sample at $\sqrt{s}=900 \mathrm{GeV}$. Results are shown for events selected with the corrected charged-track multiplicity cutoff $n_{\mathrm{ch}}>10$ (detector level) and $n_{\mathrm{ch}}^{\mathrm{gen}}>10$ (particle level) (* indicates before the $\max \left(p_{T}\right)$ cut correction).

\begin{tabular}{lccc}
\hline \hline Model(tune) & \multicolumn{3}{c}{ PYTHIA6(MC09) } \\
\hline low $p_{T}$ cut & \multicolumn{2}{c}{$>100 \mathrm{MeV}$} & $>500 \mathrm{MeV}$ \\
$\max \left(p_{T}\right)$ cut & $<10 \mathrm{GeV}$ & $<1 \mathrm{GeV}$ & $<10 \mathrm{GeV}$ \\
& $n_{\mathrm{ch}}>10$ & $($ corrected detector level) \\
$N_{\text {ch }}^{\text {gen }}$ & 21.91 & $15.28^{*}$ & 15.2 \\
diffractive/total & $3.8( \pm 0.1) \%$ & $14.7( \pm 0.2) \%$ & $<0.01 \%$ \\
& \multicolumn{2}{c}{$\mathrm{n}_{\mathrm{ch}}^{\text {gen }}>10$ (particle level) } \\
$N_{\text {ch }}^{\text {gen }}$ & 21.45 & 15.47 & 15.75 \\
diffractive/total & $3.7( \pm 0.1) \%$ & $13.8( \pm 0.2) \%$ & $<0.01 \%$ \\
\hline \hline
\end{tabular}



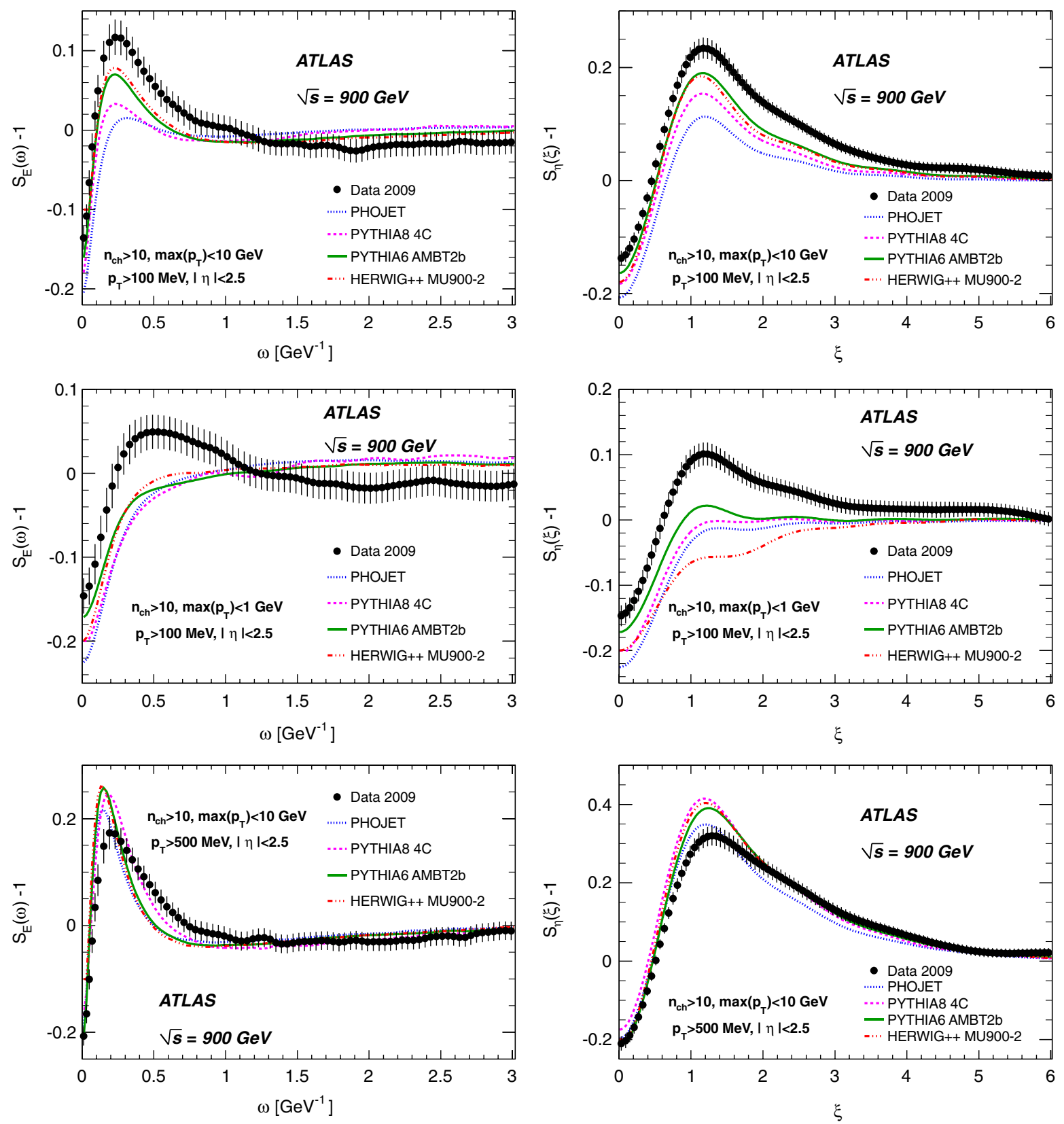

FIG. 9 (color online). Corrected data compared to particle-level predictions from various MC models using conventional hadronization algorithms. The left and right plots are for the $S_{E}$ and $S_{\eta}$ power spectra, respectively. Top: inclusive event selection $\left(p_{T}>100 \mathrm{MeV}, \max \left(p_{T}\right)<10 \mathrm{GeV}\right)$. Middle: event selection with enhanced soft component $\left(p_{T}>100 \mathrm{MeV}\right.$, $\left.\max \left(p_{T}\right)<1 \mathrm{GeV}\right)$. Bottom: event selection with reduced soft component $\left(p_{T}>500 \mathrm{MeV}, \max \left(p_{T}\right)<10 \mathrm{GeV}\right)$. ATLAS data collected at $\sqrt{s}=900 \mathrm{GeV}$ and selected with $n_{\mathrm{ch}}>10$. The error bars correspond to the combined statistical and systematic uncertainties.

average corrected charged-track multiplicity are shown in Table IV. For comparison, the average charged-particle multiplicity of PYTHIA6 MC09 samples is shown in Table V, together with the fraction of diffractive events in the sample (the estimate is based on the nominal cross section obtained from the generator). The corrected data are compared to the particle-level prediction of various models in Fig. 9.

\section{APPENDIX B}

The deconvolution technique employed in this analysis is a model-independent procedure suitable for observables with linear dependence on the track reconstruction efficiency. The dependence of the shape of the measured distribution on the fraction of reconstructed tracks is studied by convolution with the track reconstruction 


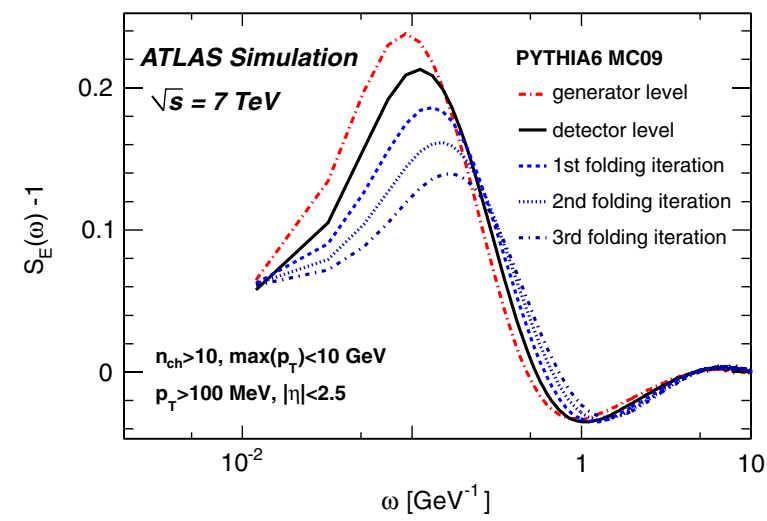

FIG. 10 (color online). Power spectrum calculated at the generator level, detector level and following three folding iterations as described in the text. Secondary tracks are removed from the samples in Figs. 10 and 11.

efficiency matrix ("double-folding"): the reconstructed tracks are randomly rejected from the sample according to the parametrized reconstruction efficiency and the power spectra are recalculated. The procedure is repeated two more times, so that a sequence of three folding iterations is available for each measured distribution. The folding iterations, together with the measured distribution and the deconvoluted (MC truth) distribution, obey simple scaling rules:

(i) the size of correlations (along the $S-1$ axis) scales linearly with the multiplicity (physically it depends on the fraction of correlated pairs which have quadratic multiplicity dependence, partially compensated by the normalization factor in Eqs. (1) and (4)).

(ii) the shape of the power spectrum $S_{E}$ scales linearly in $\omega$ with the fraction of energy removed from the hadron chain (in $S_{\eta}$, the position of the peak stays nearly constant).

A typical example of the variation of the power spectrum with the number of applied folding iterations is shown in Fig. 10 on a PYTHIA6 sample with inclusive event selection. For better illustration of the scaling symmetry, secondary tracks are removed from the sample using the generator level information.

The deconvolution of the power spectrum requires finding a set of scaling factors $f_{y}, f_{x}(x=\omega, \xi)$ which fulfill the requirement

$$
\begin{array}{r}
S_{i-1}(x)-1=\frac{1}{f_{y}}\left[S_{i}\left(\frac{x}{f_{x}}\right)-1\right], \\
0<x<10, \quad 0<i<4,
\end{array}
$$

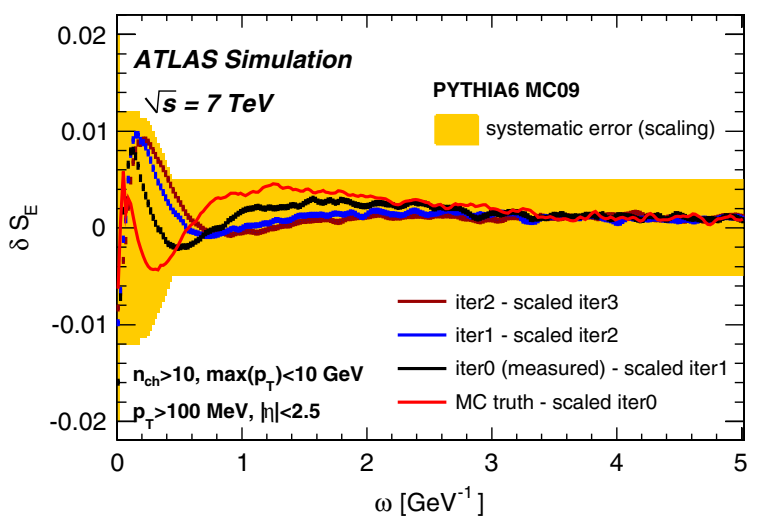

FIG. 11 (color online). The closure test of the scaling deconvolution method. Follow-up of the scaling precision over several folding iterations allows to verify the stability and to estimate the associated systematic error.

where $S_{i}$ designates the $i$-th folding iteration of the power $\operatorname{spectrum}(i=0$ for uncorrected data, -1 for corrected data).

The origin of observed correlations may be diverse, with variable dependence on the track reconstruction efficiency. A better precision can therefore be obtained by splitting the observed distribution empirically into components and by estimating the scaling factors per component.

The power spectra calculated in this paper can, to good approximation, be fit with a combination of a Landau distribution [28] (to describe the resonant peak structure), an exponentially falling background (driven by local momentum conservation of adjacent hadron pairs) and a polynomial of first or second degree to describe the upper tail of the distribution. In practice, the subtraction of a single background term and determination of 2-3 effective scaling factors is sufficient to describe the difference between a pair of folding iterations; the replacement of the $f_{x}$ with an attenuated scaling factor $f_{x} \rightarrow f_{x}^{\prime}=$ $\left(f_{x}+x\right) /(1+x)$ helps smooth the transition into the upper part of the spectrum.

The knowledge of a single pair of consecutive iterations is sufficient to calculate the scaling factors. Typically, they are determined using the difference between uncorrected data and the first folding iteration $(1 \rightarrow 0)$. The stability of the scaling factors is verified by applying them on the folding iterations of higher orders $(2 \rightarrow 1,3 \rightarrow 2)$ while the residual discrepancies serve as a basis for the estimate of the associated systematic uncertainty (Fig. 11). The deconvoluted power spectrum is obtained with the help of Eq. (B1) as the extrapolated $(0 \rightarrow-1)$ member of the sequence of folding iterations. 
[1] ATLAS Collaboration, Phys. Lett. B 688, 21 (2010).

[2] ATLAS Collaboration, New J. Phys. 13, 053033 (2011).

[3] ALICE Collaboration, Eur. Phys. J. C 65, 111 (2010).

[4] ALICE Collaboration, Eur. Phys. J. C 68, 89 (2010).

[5] CMS Collaboration, J. High Energy Phys. 02 (2010) 041.

[6] CMS Collaboration, Phys. Rev. Lett. 105, 022002 (2010).

[7] CMS Collaboration, J. High Energy Phys. 01 (2011) 079.

[8] Y. I. Azimov et al., Z. Phys. C 27, 65 (1985).

[9] B. Andersson et al., Phys. Rep. 97, 31 (1983).

[10] B. R. Webber, Nucl. Phys. B238, 492 (1984).

[11] B. Andersson et al., J. High Energy Phys. 09 (1998) 014.

[12] The ATLAS reference system is a right-handed coordinate system with its origin at the nominal interaction point at the center of the detector. Cylindrical coordinates $(r, \phi)$ are used in the transverse plane, $\phi$ being the azimuthal angle around the beam axis. The pseudorapidity is defined in terms of the polar angle $\theta$ as $\eta=-\ln \tan (\theta / 2)$.

[13] Š. Todorova, in Proceedings of the XL International Symposium on Multiparticle Dynamics, Antwerp, 2010, edited by $\mathrm{P}$. van Mechelen and N. van Remortel (Uitgeverij UPA University Press, Antwerp, 2011).

[14] ATLAS Collaboration, JINST 3, S08003 (2008).

[15] T. Sjöstrand, S. Mrenna, and P.Z. Skands, J. High Energy Phys. 05 (2006) 026.
[16] ATLAS Collaboration, Report No. ATL-PHYS-PUB2010-002, 2010, https://cdsweb.cern.ch/record/1247375.

[17] GEANT4 Collaboration, Nucl. Instrum. Methods Phys. Res., Sect. A 506, 250 (2003).

[18] ATLAS Collaboration, Eur. Phys. J. C 70, 823 (2010).

[19] R. Engel, Z. Phys. C 66, 203 (1995).

[20] M. Bahr et al., Eur. Phys. J. C 58, 639 (2008).

[21] http://projects.hepforge.org/herwig/trac/wiki/ MB_UE_tunes.

[22] T. Sjöstrand, S. Mrenna, and P.Z. Skands, Comput. Phys. Commun. 178, 852 (2008).

[23] ATLAS Collaboration, Report No. ATL-PHYS-PUB2011-009, 2011, https://cdsweb.cern.ch/record/1363300.

[24] http://projects.hepforge.org/helix/.

[25] Primary particles are defined as all particles with lifetime longer than $0.3 \times 10^{-10} \mathrm{~s}$ originating from the primary interaction or from subsequent decay of particles with shorter lifetime.

[26] The track reconstruction efficiency is parametrized as a function of track $p_{T}$ and pseudorapidity $\eta$.

[27] J. W. Monk and C. Oropeza-Barrera, arXiv:1111.4896v2 [NIM (to be published)].

[28] Landau(MPV, $\sigma)=\frac{1}{\pi} \int_{0}^{\infty} e^{-u \ln u-\lambda u} \sin \pi u \mathrm{du}$, where $\lambda=$ $\frac{\omega-\mathrm{MPV}}{\sigma}$.

G. Aad, ${ }^{47}$ B. Abbott, ${ }^{109}$ J. Abdallah, ${ }^{11}$ A. A. Abdelalim, ${ }^{48}$ A. Abdesselam,,${ }^{116}$ O. Abdinov,${ }^{10}$ B. Abi,,${ }^{110}$ M. Abolins, ${ }^{86}$ O. S. AbouZeid, ${ }^{156}$ H. Abramowicz, ${ }^{151}$ H. Abreu, ${ }^{113}$ E. Acerbi, ${ }^{87 a, 87 b}$ B. S. Acharya, ${ }^{162 a, 162 b}$ L. Adamczyk,${ }^{37}$ D. L. Adams, ${ }^{24}$ T. N. Addy, ${ }^{55}$ J. Adelman, ${ }^{173}$ M. Aderholz, ${ }^{97}$ S. Adomeit,${ }^{96}$ P. Adragna, ${ }^{73}$ T. Adye, ${ }^{127}$ S. Aefsky, ${ }^{22}$ J. A. Aguilar-Saavedra, ${ }^{122 b, b}$ M. Aharrouche ${ }^{79}$ S. P. Ahlen,${ }^{21}$ F. Ahles,${ }^{47}$ A. Ahmad, ${ }^{146}$ M. Ahsan, ${ }^{40}$

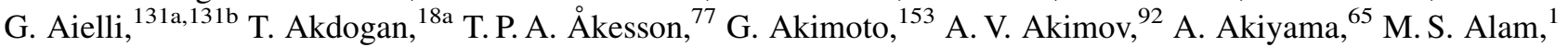
M. A. Alam,${ }^{74}$ J. Albert, ${ }^{167}$ S. Albrand,${ }^{54}$ M. Aleksa, ${ }^{29}$ I. N. Aleksandrov, ${ }^{63}$ F. Alessandria ${ }^{87 a}$ C. Alexa, ${ }^{25 a}$ G. Alexander, ${ }^{151}$ G. Alexandre, ${ }^{48}$ T. Alexopoulos, ${ }^{9}$ M. Alhroob,${ }^{20}$ M. Aliev, ${ }^{15}$ G. Alimonti, ${ }^{87 a}$ J. Alison,,${ }^{118}$ M. Aliyev, ${ }^{10}$ P. P. Allport, ${ }^{71}$ S. E. Allwood-Spiers,${ }^{52}$ J. Almond, ${ }^{80}$ A. Aloisio, ${ }^{100 a, 100 b}$ R. Alon, ${ }^{169}$ A. Alonso, ${ }^{77}$ B. Alvarez Gonzalez, ${ }^{86}$ M. G. Alviggi, ${ }^{100 a, 100 b}$ K. Amako, ${ }^{64}$ P. Amaral, ${ }^{29}$ C. Amelung, ${ }^{22}$ V. V. Ammosov, ${ }^{126}$ A. Amorim, ${ }^{122 a, c}$ G. Amorós, ${ }^{165}$ N. Amram, ${ }^{151}$ C. Anastopoulos, ${ }^{29}$ L. S. Ancu, ${ }^{16}$ N. Andari, ${ }^{113}$ T. Andeen, ${ }^{34}$ C. F. Anders, ${ }^{20}$ G. Anders,${ }^{57 a}$ K. J. Anderson, ${ }^{30}$ A. Andreazza, ${ }^{87 a, 87 b}$ V. Andrei,${ }^{57 a}$ M-L. Andrieux,${ }^{54}$

X. S. Anduaga, ${ }^{68}$ A. Angerami, ${ }^{34}$ F. Anghinolfi, ${ }^{29}$ A. Anisenkov, ${ }^{105}$ N. Anjos, ${ }^{122 a}$ A. Annovi, ${ }^{46}$ A. Antonaki, ${ }^{8}$ M. Antonelli, ${ }^{46}$ A. Antonov, ${ }^{94}$ J. Antos,${ }^{142 b}$ F. Anulli, ${ }^{130 a}$ S. Aoun,${ }^{81}$ L. Aperio Bella, ${ }^{4}$ R. Apolle, ${ }^{116, d}$ G. Arabidze,${ }^{86}$ I. Aracena ${ }^{141}$ Y. Arai, ${ }^{64}$ A. T. H. Arce, ${ }^{44}$ J. P. Archambault,${ }^{28}$ S. Arfaoui, ${ }^{146}$ J-F. Arguin, ${ }^{14}$ E. Arik, ${ }^{18 a, a}$ M. Arik ${ }^{18 a}$ A. J. Armbruster, ${ }^{85}$ O. Arnaez, ${ }^{79}$ C. Arnault, ${ }^{113}$ A. Artamonov, ${ }^{93}$ G. Artoni, ${ }^{130 a, 130 b}$ D. Arutinov,${ }^{20}$ S. Asai, ${ }^{153}$ R. Asfandiyarov, ${ }^{170}$ S. Ask, ${ }^{27}$ B. Asman, ${ }^{144 a, 144 b}$ L. Asquith,${ }^{5}$ K. Assamagan, ${ }^{24}$ A. Astbury, ${ }^{167}$ A. Astvatsatourov, ${ }^{51}$ B. Aubert, ${ }^{4}$ E. Auge,${ }^{113}$ K. Augsten, ${ }^{125}$ M. Aurousseau, ${ }^{143 a}$ G. Avolio, ${ }^{161}$ R. Avramidou, ${ }^{9}$ D. Axen,,${ }^{166}$ C. Ay,${ }^{53}$ G. Azuelos, ${ }^{91, \mathrm{e}}$ Y. Azuma, ${ }^{153}$ M. A. Baak, ${ }^{29}$ G. Baccaglioni, ${ }^{87 a}$ C. Bacci, ${ }^{132 a, 132 b}$ A. M. Bach, ${ }^{14}$ H. Bachacou, ${ }^{134}$ K. Bachas, ${ }^{29}$ G. Bachy, ${ }^{29}$ M. Backes ${ }^{48}$ M. Backhaus, ${ }^{20}$ E. Badescu, ${ }^{25 a}$ P. Bagnaia, ${ }^{130 a, 130 b}$ S. Bahinipati, ${ }^{2}$ Y. Bai, ${ }^{32 a}$ D. C. Bailey, ${ }^{156}$ T. Bain, ${ }^{156}$ J. T. Baines, ${ }^{127}$ O. K. Baker,${ }^{173}$ M. D. Baker, ${ }^{24}$ S. Baker, ${ }^{75}$ E. Banas, ${ }^{38}$ P. Banerjee, ${ }^{91}$ Sw. Banerjee, ${ }^{170}$ D. Banfi, ${ }^{29}$ A. Bangert, ${ }^{148}$ V. Bansal, ${ }^{167}$ H. S. Bansil, ${ }^{17}$ L. Barak, ${ }^{169}$ S. P. Baranov, ${ }^{92}$ A. Barashkou, ${ }^{63}$ A. Barbaro Galtieri, ${ }^{14}$ T. Barber, ${ }^{47}$ E. L. Barberio, ${ }^{84}$ D. Barberis, ${ }^{49 a, 49 b}$ M. Barbero, ${ }^{20}$ D. Y. Bardin, ${ }^{63}$ T. Barillari, ${ }^{97}$ M. Barisonzi, ${ }^{172}$ T. Barklow, ${ }^{141}$ N. Barlow, ${ }^{27}$ B. M. Barnett,,${ }^{127}$ R. M. Barnett,${ }^{14}$ A. Baroncelli, ${ }^{132 a}$ G. Barone, ${ }^{48}$ A. J. Barr, ${ }^{116}$ F. Barreiro, ${ }^{78}$ J. Barreiro Guimarães da Costa, ${ }^{56}$ P. Barrillon, ${ }^{113}$ R. Bartoldus, ${ }^{141}$ A. E. Barton, ${ }^{69}$ V. Bartsch, ${ }^{147}$ R. L. Bates,${ }^{52}$ L. Batkova, ${ }^{142 a}$ J. R. Batley, ${ }^{27}$ A. Battaglia, ${ }^{16}$ M. Battistin, ${ }^{29}$ F. Bauer, ${ }^{134}$ H. S. Bawa, ${ }^{141, f}$ S. Beale, ${ }^{96}$ B. Beare, ${ }^{156}$ T. Beau, ${ }^{76}$ P. H. Beauchemin, ${ }^{159}$ R. Beccherle, ${ }^{49 a}$ P. Bechtle, ${ }^{20}$ H. P. Beck, ${ }^{16}$ S. Becker,${ }^{96}$ M. Beckingham, ${ }^{136}$ K. H. Becks, ${ }^{172}$ A. J. Beddall, ${ }^{18 c}$ A. Beddall, ${ }^{18 c}$ S. Bedikian, ${ }^{173}$ V. A. Bednyakov, ${ }^{63}$ C. P. Bee ${ }^{81}$ M. Begel,${ }^{24}$ S. Behar Harpaz, ${ }^{150}$ P. K. Behera, ${ }^{61}$ M. Beimforde, ${ }^{97}$ C. Belanger-Champagne, ${ }^{83}$ P. J. Bell, ${ }^{48}$ 
W. H. Bell, ${ }^{48}$ G. Bella, ${ }^{151}$ L. Bellagamba, ${ }^{19 a}$ F. Bellina, ${ }^{29}$ M. Bellomo, ${ }^{29}$ A. Belloni, ${ }^{56}$ O. Beloborodova, ${ }^{105, g}$ K. Belotskiy, ${ }^{94}$ O. Beltramello, ${ }^{29}$ S. Ben Ami,${ }^{150}$ O. Benary,${ }^{151}$ D. Benchekroun, ${ }^{133 a}$ C. Benchouk,${ }^{81}$ M. Bendel,${ }^{79}$ N. Benekos, ${ }^{163}$ Y. Benhammou, ${ }^{151}$ E. Benhar Noccioli, ${ }^{48}$ J. A. Benitez Garcia, ${ }^{157 b}$ D. P. Benjamin, ${ }^{44}$ M. Benoit, ${ }^{113}$ J. R. Bensinger, ${ }^{22}$ K. Benslama, ${ }^{128}$ S. Bentvelsen, ${ }^{103}$ D. Berge, ${ }^{29}$ E. Bergeaas Kuutmann, ${ }^{41}$ N. Berger, ${ }^{4}$ F. Berghaus, ${ }^{167}$ E. Berglund, ${ }^{103}$ J. Beringer,${ }^{14}$ P. Bernat,${ }^{75}$ R. Bernhard ${ }^{47}$ C. Bernius,${ }^{24}$ T. Berry, ${ }^{74}$ C. Bertella,${ }^{81}$ A. Bertin, ${ }^{19 a, 19 b}$ F. Bertinelli, ${ }^{29}$ F. Bertolucci, ${ }^{120 a, 120 b}$ M. I. Besana, ${ }^{87 a, 87 b}$ N. Besson, ${ }^{134}$ S. Bethke, ${ }^{97}$ W. Bhimji, ${ }^{45}$ R. M. Bianchi, ${ }^{29}$ M. Bianco, ${ }^{70 a, 70 b}$ O. Biebel, ${ }^{96}$ S. P. Bieniek,${ }^{75}$ K. Bierwagen, ${ }^{53}$ J. Biesiada, ${ }^{14}$ M. Biglietti, ${ }^{132 a}$ H. Bilokon, ${ }^{46}$ M. Bindi, ${ }^{19 a, 19 b}$ S. Binet, ${ }^{113}$ A. Bingul, ${ }^{18 \mathrm{c}}$ C. Bini, ${ }^{130 a, 130 b}$ C. Biscarat, ${ }^{175}$ U. Bitenc, ${ }^{47}$ K. M. Black, ${ }^{21}$ R. E. Blair, ${ }^{5}$ J.-B. Blanchard, ${ }^{134}$ G. Blanchot,${ }^{29}$ T. Blazek, ${ }^{142 a}$ C. Blocker,${ }^{22}$ J. Blocki, ${ }^{38}$ A. Blondel,${ }^{48}$ W. Blum, ${ }^{79}$

U. Blumenschein, ${ }^{53}$ G. J. Bobbink, ${ }^{103}$ V. B. Bobrovnikov, ${ }^{105}$ S. S. Bocchetta, ${ }^{77}$ A. Bocci,${ }^{44}$ C. R. Boddy,${ }^{116}$ M. Boehler,${ }^{41}$ J. Boek, ${ }^{172}$ N. Boelaert,${ }^{35}$ S. Böser, ${ }^{75}$ J. A. Bogaerts, ${ }^{29}$ A. Bogdanchikov, ${ }^{105}$ A. Bogouch, ${ }^{88, a}$ C. Bohm, ${ }^{144 a}$ V. Boisvert, ${ }^{74}$ T. Bold,${ }^{37}$ V. Boldea, ${ }^{25 a}$ N. M. Bolnet, ${ }^{134}$ M. Bona ${ }^{73}$ V. G. Bondarenko, ${ }^{94}$ M. Bondioli, ${ }^{161}$ M. Boonekamp, ${ }^{134}$ G. Boorman, ${ }^{74}$ C. N. Booth, ${ }^{137}$ S. Bordoni, ${ }^{76}$ C. Borer, ${ }^{16}$ A. Borisov, ${ }^{126}$ G. Borissov, ${ }^{69}$ I. Borjanovic, ${ }^{12 \mathrm{a}} \mathrm{S}$. Borroni, ${ }^{85} \mathrm{~K}$. Bos, ${ }^{103} \mathrm{D}$. Boscherini, ${ }^{19 \mathrm{a}} \mathrm{M}$. Bosman, ${ }^{11} \mathrm{H}$. Boterenbrood, ${ }^{103}$ D. Botterill, ${ }^{127}$ J. Bouchami, ${ }^{91}$ J. Boudreau, ${ }^{121}$ E. V. Bouhova-Thacker ${ }^{69}$ D. Boumediene, ${ }^{33}$ C. Bourdarios, ${ }^{113}$

N. Bousson, ${ }^{81}$ A. Boveia, ${ }^{30}$ J. Boyd, ${ }^{29}$ I. R. Boyko, ${ }^{63}$ N. I. Bozhko, ${ }^{126}$ I. Bozovic-Jelisavcic, ${ }^{12 b}$ J. Bracinik, ${ }^{17}$ A. Braem, ${ }^{29}$ P. Branchini, ${ }^{132 a}$ G. W. Brandenburg, ${ }^{56}$ A. Brandt, ${ }^{7}$ G. Brandt,,${ }^{116}$ O. Brandt, ${ }^{53}$ U. Bratzler, ${ }^{154}$ B. Brau, ${ }^{82}$ J. E. Brau, ${ }^{112}$ H. M. Braun, ${ }^{172}$ B. Brelier, ${ }^{156}$ J. Bremer, ${ }^{29}$ R. Brenner, ${ }^{164}$ S. Bressler, ${ }^{169}$ D. Breton, ${ }^{113}$ D. Britton, ${ }^{52}$ F. M. Brochu, ${ }^{27}$ I. Brock, ${ }^{20}$ R. Brock,${ }^{86}$ T. J. Brodbeck, ${ }^{69}$ E. Brodet,${ }^{151}$ F. Broggi, ${ }^{87 a}$ C. Bromberg, ${ }^{86}$ J. Bronner, ${ }^{97}$

G. Brooijmans, ${ }^{34}$ W. K. Brooks, ${ }^{31 b}$ G. Brown, ${ }^{80}$ H. Brown, ${ }^{7}$ P. A. Bruckman de Renstrom, ${ }^{38}$ D. Bruncko, ${ }^{142 b}$

R. Bruneliere, ${ }^{47}$ S. Brunet, ${ }^{59}$ A. Bruni,${ }^{19 a}$ G. Bruni, ${ }^{19 a}$ M. Bruschi,${ }^{19 a}$ T. Buanes,${ }^{13}$ Q. Buat,${ }^{54}$ F. Bucci,${ }^{48}$

J. Buchanan, ${ }^{116}$ N. J. Buchanan, ${ }^{2}$ P. Buchholz ${ }^{139}$ R. M. Buckingham, ${ }^{116}$ A. G. Buckley, ${ }^{45}$ S. I. Buda, ${ }^{25 a}$ I. A. Budagov ${ }^{63}$ B. Budick, ${ }^{106}$ V. Büscher ${ }^{79}$ L. Bugge,${ }^{115}$ O. Bulekov, ${ }^{94}$ M. Bunse, ${ }^{42}$ T. Buran,${ }^{115}$ H. Burckhart,${ }^{29}$

S. Burdin ${ }^{71}$ T. Burgess, ${ }^{13}$ S. Burke, ${ }^{127}$ E. Busato, ${ }^{33}$ P. Bussey,${ }^{52}$ C. P. Buszello, ${ }^{164}$ F. Butin, ${ }^{29}$ B. Butler, ${ }^{141}$ J. M. Butler, ${ }^{21}$ C. M. Buttar, ${ }^{52}$ J. M. Butterworth, ${ }^{75}$ W. Buttinger, ${ }^{27}$ S. Cabrera Urbán, ${ }^{165}$ D. Caforio, ${ }^{19 a, 19 b}$ O. Cakir, ${ }^{3 a}$ P. Calafiura, ${ }^{14}$ G. Calderini, ${ }^{76}$ P. Calfayan, ${ }^{96}$ R. Calkins, ${ }^{104}$ L. P. Caloba, ${ }^{23 a}$ R. Caloi, ${ }^{130 a, 130 b}$ D. Calvet, ${ }^{33}$ S. Calvet, ${ }^{33}$ R. Camacho Toro, ${ }^{33}$ P. Camarri, ${ }^{131 \mathrm{a}, 131 \mathrm{~b}}$ M. Cambiaghi, ${ }^{117 \mathrm{a}, 117 \mathrm{~b}}$ D. Cameron, ${ }^{115}$ L. M. Caminada, ${ }^{14}$ S. Campana, ${ }^{29}$ M. Campanelli, ${ }^{75}$ V. Canale, ${ }^{100 a, 100 b}$ F. Canelli, ${ }^{30, \mathrm{~h}}$ A. Canepa, ${ }^{157 \mathrm{a}}$ J. Cantero,${ }^{78}$ L. Capasso, ${ }^{100 a, 100 b}$

M. D. M. Capeans Garrido, ${ }^{29}$ I. Caprini, ${ }^{25 a}$ M. Caprini ${ }^{25 a}$ D. Capriotti, ${ }^{97}$ M. Capua, ${ }^{36 a, 36 b}$ R. Caputo, ${ }^{79}$ C. Caramarcu, ${ }^{24}$ R. Cardarelli, ${ }^{131 \mathrm{a}}$ T. Carli, ${ }^{29}$ G. Carlino, ${ }^{100 \mathrm{a}}$ L. Carminati, ${ }^{87 \mathrm{a}, 87 \mathrm{~b}}$ B. Caron, ${ }^{83}$ S. Caron, ${ }^{102}$ G. D. Carrillo Montoya, ${ }^{170}$ A. A. Carter ${ }^{73}$ J. R. Carter, ${ }^{27}$ J. Carvalho, ${ }^{122 a, i}$ D. Casadei, ${ }^{106}$ M. P. Casado, ${ }^{11}$ M. Cascella, ${ }^{120 a, 120 b}$ C. Caso, ${ }^{49 a, 49 b, a}$ A. M. Castaneda Hernandez, ${ }^{170}$ E. Castaneda-Miranda, ${ }^{170}$

V. Castillo Gimenez, ${ }^{165}$ N. F. Castro, ${ }^{122 a}$ G. Cataldi, ${ }^{70 a}$ F. Cataneo,${ }^{29}$ A. Catinaccio, ${ }^{29}$ J. R. Catmore, ${ }^{29}$ A. Cattai, ${ }^{29}$ G. Cattani, ${ }^{131 a, 131 b}$ S. Caughron, ${ }^{86}$ D. Cauz, ${ }^{162 a, 162 c}$ P. Cavalleri, ${ }^{76}$ D. Cavalli, ${ }^{87 a}$ M. Cavalli-Sforza, ${ }^{11}$ V. Cavasinni, ${ }^{120 a, 120 b}$ F. Ceradini, ${ }^{132 a, 132 b}$ A. S. Cerqueira, ${ }^{23 b}$ A. Cerri, ${ }^{29}$ L. Cerrito, ${ }^{73}$ F. Cerutti, ${ }^{46}$ S. A. Cetin, ${ }^{18 b}$ F. Cevenini, ${ }^{100 a, 100 b}$ A. Chafaq, ${ }^{133 a}$ D. Chakraborty, ${ }^{104}$ K. Chan, ${ }^{2}$ B. Chapleau, ${ }^{83}$ J. D. Chapman, ${ }^{27}$ J. W. Chapman, ${ }^{85}$ E. Chareyre, ${ }^{76}$ D. G. Charlton, ${ }^{17}$ V. Chavda,${ }^{80}$ C. A. Chavez Barajas, ${ }^{29}$ S. Cheatham, ${ }^{83}$ S. Chekanov, ${ }^{5}$

S. V. Chekulaev, ${ }^{157 a}$ G. A. Chelkov, ${ }^{63}$ M. A. Chelstowska, ${ }^{102}$ C. Chen,${ }^{62}$ H. Chen,${ }^{24}$ S. Chen, ${ }^{32 c}$ T. Chen, ${ }^{32 c}$ X. Chen, ${ }^{170}$ S. Cheng, ${ }^{32 a}$ A. Cheplakov, ${ }^{63}$ V. F. Chepurnov, ${ }^{63}$ R. Cherkaoui El Moursli, ${ }^{133 e}$ V. Chernyatin,${ }^{24}$ E. Cheu, ${ }^{6}$

S. L. Cheung, ${ }^{156}$ L. Chevalier, ${ }^{134}$ G. Chiefari, ${ }^{100 a, 100 b}$ L. Chikovani, ${ }^{50 a}$ J. T. Childers ${ }^{29}$ A. Chilingarov, ${ }^{69}$

G. Chiodini ${ }^{70 a}$ M. V. Chizhov, ${ }^{63}$ G. Choudalakis,${ }^{30}$ S. Chouridou, ${ }^{135}$ I. A. Christidi, ${ }^{75}$ A. Christov, ${ }^{47}$ D. Chromek-Burckhart, ${ }^{29}$ M. L. Chu, ${ }^{149}$ J. Chudoba,${ }^{123}$ G. Ciapetti, ${ }^{130 a, 130 b}$ K. Ciba, ${ }^{37}$ A. K. Ciftci, ${ }^{3 a}$ R. Ciftci, ${ }^{3 a}$ D. Cinca, ${ }^{33}$ V. Cindro,${ }^{72}$ M. D. Ciobotaru, ${ }^{161}$ C. Ciocca, ${ }^{19 a}$ A. Ciocio, ${ }^{14}$ M. Cirilli, ${ }^{85}$ M. Citterio, ${ }^{87 a}$ M. Ciubancan, ${ }^{25 a}$ A. Clark, ${ }^{48}$ P. J. Clark, ${ }^{45}$ W. Cleland, ${ }^{121}$ J. C. Clemens, ${ }^{81}$ B. Clement,${ }^{54}$ C. Clement,${ }^{144 a, 144 b}$ R. W. Clifft, ${ }^{127}$ Y. Coadou, ${ }^{81}$ M. Cobal, ${ }^{162 a, 162 \mathrm{c}}$ A. Coccaro, ${ }^{170}$ J. Cochran, ${ }^{62}$ P. Coe, ${ }^{116}$ J. G. Cogan, ${ }^{141}$ J. Coggeshall, ${ }^{163}$ E. Cogneras, ${ }^{175}$ J. Colas, ${ }^{4}$ A. P. Colijn, ${ }^{103}$ N. J. Collins, ${ }^{17}$ C. Collins-Tooth,${ }^{52}$ J. Collot,${ }^{54}$ G. Colon, ${ }^{82}$ P. Conde Muiño, ${ }^{122 a}$ E. Coniavitis, ${ }^{116}$ M. C. Conidi, ${ }^{11}$ M. Consonni, ${ }^{102}$ V. Consorti, ${ }^{47}$ S. Constantinescu, ${ }^{25 a}$ C. Conta, ${ }^{117 a, 117 b}$ F. Conventi, ${ }^{100 a, j}$ J. Cook,${ }^{29}$ M. Cooke, ${ }^{14}$ B. D. Cooper, ${ }^{75}$ A. M. Cooper-Sarkar, ${ }^{116}$ K. Copic, ${ }^{14}$ T. Cornelissen, ${ }^{172}$ M. Corradi, ${ }^{19 a}$ F. Corriveau, ${ }^{83, k}$ A. Cortes-Gonzalez, ${ }^{163}$ G. Cortiana, ${ }^{97}$ G. Costa, ${ }^{87 a}$ M. J. Costa, ${ }^{165}$ D. Costanzo, ${ }^{137}$ T. Costin, ${ }^{30}$ D. Côté, ${ }^{29}$ R. Coura Torres, ${ }^{23 a}$ L. Courneyea, ${ }^{167}$ G. Cowan ${ }^{74}$ C. Cowden, ${ }^{27}$ B. E. Cox,${ }^{80}$ K. Cranmer, ${ }^{106}$ F. Crescioli, ${ }^{120 \mathrm{a}, 120 \mathrm{~b}}$ M. Cristinziani, ${ }^{20}$ G. Crosetti, ${ }^{36 \mathrm{a}, 36 \mathrm{~b}}$ R. Crupi,${ }^{70 \mathrm{a}, 70 \mathrm{~b}}$ S. Crépé-Renaudin, ${ }^{54}$ 
C.-M. Cuciuc, ${ }^{25 a}$ C. Cuenca Almenar, ${ }^{173}$ T. Cuhadar Donszelmann, ${ }^{137}$ M. Curatolo, ${ }^{46}$ C. J. Curtis, ${ }^{17}$ C. Cuthbert ${ }^{148}$ P. Cwetanski, ${ }^{59}$ H. Czirr, ${ }^{139}$ P. Czodrowski, ${ }^{43}$ Z. Czyczula, ${ }^{173}$ S. D'Auria, ${ }^{52}$ M. D'Onofrio, ${ }^{71}$ A. D'Orazio, ${ }^{130 a, 130 b}$ P. V. M. Da Silva ${ }^{23 a}$ C. Da Via, ${ }^{80}$ W. Dabrowski, ${ }^{37}$ T. Dai, ${ }^{85}$ C. Dallapiccola,${ }^{82}$ M. Dam, ${ }^{35}$ M. Dameri,${ }^{49 a, 49 b}$ D. S. Damiani, ${ }^{135}$ H. O. Danielsson, ${ }^{29}$ D. Dannheim, ${ }^{97}$ V. Dao, ${ }^{48}$ G. Darbo, ${ }^{49 a}$ G. L. Darlea,${ }^{25 b}$ C. Daum,,${ }^{103}$ W. Davey, ${ }^{20}$ T. Davidek, ${ }^{124}$ N. Davidson, ${ }^{84}$ R. Davidson, ${ }^{69}$ E. Davies, ${ }^{16, d}$ M. Davies, ${ }^{91}$ A. R. Davison, ${ }^{75}$

Y. Davygora, ${ }^{57 a}$ E. Dawe, ${ }^{140}$ I. Dawson, ${ }^{137}$ J. W. Dawson, ${ }^{5, a}$ R. K. Daya-Ishmukhametova, ${ }^{22}$ K. De, ${ }^{7}$ R. de Asmundis, ${ }^{100 a}$ S. De Castro, ${ }^{19 a, 19 b}$ P. E. De Castro Faria Salgado, ${ }^{24}$ S. De Cecco, ${ }^{76}$ J. de Graat, ${ }^{96}$ N. De Groot, ${ }^{102}$ P. de Jong, ${ }^{103}$ C. De La Taille, ${ }^{113}$ H. De la Torre, ${ }^{78}$ B. De Lotto, ${ }^{162 a, 162 \mathrm{c}}$ L. de Mora, ${ }^{69}$ L. De Nooij, ${ }^{103}$ D. De Pedis, ${ }^{130 a}$ A. De Salvo, ${ }^{130 a}$ U. De Sanctis, ${ }^{162 a, 162 c}$ A. De Santo, ${ }^{147}$ J. B. De Vivie De Regie, ${ }^{113}$ S. Dean, ${ }^{75}$

W. J. Dearnaley, ${ }^{69}$ R. Debbe,${ }^{24}$ C. Debenedetti, ${ }^{45}$ D. V. Dedovich, ${ }^{63}$ J. Degenhardt, ${ }^{118}$ M. Dehchar, ${ }^{116}$ C. Del Papa ${ }^{162 a, 162 \mathrm{c}}$ J. Del Peso,${ }^{78}$ T. Del Prete, ${ }^{120 a, 120 \mathrm{~b}}$ T. Delemontex,${ }^{54}$ M. Deliyergiyev, ${ }^{72}$ A. Dell' Acqua ${ }^{29}$ L. Dell'Asta, ${ }^{21}$ M. Della Pietra, ${ }^{100 a, j}$ D. della Volpe,${ }^{100 a, 100 b}$ M. Delmastro, ${ }^{4}$ N. Delruelle ${ }^{29}$ P. A. Delsart,${ }^{54}$ C. Deluca, ${ }^{146}$ S. Demers, ${ }^{173}$ M. Demichev, ${ }^{63}$ B. Demirkoz,${ }^{11,1}$ J. Deng,${ }^{161}$ S. P. Denisov, ${ }^{126}$ D. Derendarz,${ }^{38}$ J. E. Derkaoui, ${ }^{133 d}$ F. Derue,${ }^{76}$ P. Dervan,${ }^{71}$ K. Desch, ${ }^{20}$ E. Devetak, ${ }^{146}$ P. O. Deviveiros, ${ }^{103}$ A. Dewhurst, ${ }^{127}$ B. DeWilde, ${ }^{146}$ S. Dhaliwal, ${ }^{156}$ R. Dhullipudi, ${ }^{24, m}$ A. Di Ciaccio, ${ }^{131 a, 131 b}$ L. Di Ciaccio, ${ }^{4}$ A. Di Girolamo, ${ }^{29}$ B. Di Girolamo, ${ }^{29}$ S. Di Luise, ${ }^{132 a, 132 b}$ A. Di Mattia, ${ }^{170}$ B. Di Micco, ${ }^{29}$ R. Di Nardo,${ }^{46}$ A. Di Simone, ${ }^{131 a, 131 b}$ R. Di Sipio, ${ }^{19 a, 19 b}$ M. A. Diaz, ${ }^{31 a}$ F. Diblen, ${ }^{18 c}$ E. B. Diehl,${ }^{85}$ J. Dietrich, ${ }^{41}$ T. A. Dietzsch,${ }^{57 a}$ S. Diglio, ${ }^{84}$ K. Dindar Yagci, ${ }^{39}$ J. Dingfelder, ${ }^{20}$ C. Dionisi, ${ }^{130 a, 130 b}$ P. Dita, ${ }^{25 a}$ S. Dita, ${ }^{25 a}$ F. Dittus, ${ }^{29}$ F. Djama, ${ }^{81}$ T. Djobava, ${ }^{50 b}$ M. A. B. do Vale, ${ }^{23 c}$ A. Do Valle Wemans, ${ }^{122 a}$ T. K. O. Doan, ${ }^{4}$ M. Dobbs,${ }^{83}$ R. Dobinson, ${ }^{29, a}$ D. Dobos,${ }^{29}$ E. Dobson, ${ }^{29, n}$ J. Dodd,${ }^{34}$ C. Doglioni,${ }^{48}$ T. Doherty,${ }^{52}$ Y. Doi, ${ }^{64, a}$ J. Dolejsi, ${ }^{124}$ I. Dolenc, ${ }^{72}$ Z. Dolezal, ${ }^{124}$ B. A. Dolgoshein, ${ }^{94, a}$ T. Dohmae, ${ }^{153}$ M. Donadelli, ${ }^{23 d}$ M. Donega, ${ }^{118}$ J. Donini, ${ }^{33}$ J. Dopke, ${ }^{29}$ A. Doria, ${ }^{100 a}$ A. Dos Anjos, ${ }^{170}$ M. Dosil, ${ }^{11}$ A. Dotti, ${ }^{120 a, 120 b}$ M. T. Dova,${ }^{68}$ J. D. Dowell, ${ }^{17}$ A. D. Doxiadis, ${ }^{103}$ A. T. Doyle, ${ }^{52}$ Z. Drasal, ${ }^{124}$ J. Drees, ${ }^{172}$ N. Dressnandt, ${ }^{118}$ H. Drevermann, ${ }^{29}$ C. Driouichi, ${ }^{35}$ M. Dris, ${ }^{9}$ J. Dubbert, ${ }^{97}$ S. Dube, ${ }^{14}$ E. Duchovni, ${ }^{169}$ G. Duckeck, ${ }^{96}$ A. Dudarev, ${ }^{29}$ F. Dudziak, ${ }^{62}$ M. Dührssen,${ }^{29}$ I. P. Duerdoth,${ }^{80}$ L. Duflot,${ }^{113}$ M-A. Dufour, ${ }^{83}$ M. Dunford, ${ }^{29}$ H. Duran Yildiz,${ }^{3 a}$ R. Duxfield, ${ }^{137}$ M. Dwuznik, ${ }^{37}$ F. Dydak,${ }^{29}$ M. Düren, ${ }^{51}$ W. L. Ebenstein, ${ }^{44}$ J. Ebke, ${ }^{96}$ S. Eckweiler, ${ }^{79}$ K. Edmonds, ${ }^{79}$ C. A. Edwards, ${ }^{74}$ N. C. Edwards,${ }^{52}$ W. Ehrenfeld ${ }^{41}$ T. Ehrich, ${ }^{97}$ T. Eifert, ${ }^{141}$ G. Eigen, ${ }^{13}$ K. Einsweiler, ${ }^{14}$ E. Eisenhandler, ${ }^{73}$ T. Ekelof, ${ }^{164}$ M. El Kacimi, ${ }^{133 c}$ M. Ellert,,${ }^{164}$ S. Elles, ${ }^{4}$ F. Ellinghaus, ${ }^{79}$ K. Ellis, ${ }^{73}$ N. Ellis, ${ }^{29}$ J. Elmsheuser, ${ }^{96}$ M. Elsing, ${ }^{29}$ D. Emeliyanov, ${ }^{127}$ R. Engelmann, ${ }^{146}$ A. Engl, ${ }^{96}$ B. Epp ${ }^{60}$ A. Eppig, ${ }^{85}$ J. Erdmann, ${ }^{53}$ A. Ereditato, ${ }^{16}$ D. Eriksson, ${ }^{144 a}$ J. Ernst, ${ }^{1}$ M. Ernst,${ }^{24}$ J. Ernwein, ${ }^{134}$ D. Errede,${ }^{163}$ S. Errede, ${ }^{163}$ E. Ertel, ${ }^{79}$ M. Escalier, ${ }^{113}$ C. Escobar, ${ }^{121}$ X. Espinal Curull, ${ }^{11}$ B. Esposito, ${ }^{46}$ F. Etienne ${ }^{81}$ A. I. Etienvre, ${ }^{134}$ E. Etzion, ${ }^{151}$ D. Evangelakou, ${ }^{53}$ H. Evans,${ }^{59}$ L. Fabbri, ${ }^{19 a}, 19 \mathrm{~b}$ C. Fabre,${ }^{29}$ R. M. Fakhrutdinov, ${ }^{126}$ S. Falciano, ${ }^{130 a}$ Y. Fang, ${ }^{170}$ M. Fanti, ${ }^{87 a, 87 b}$ A. Farbin, ${ }^{7}$ A. Farilla, ${ }^{132 a}$ J. Farley, ${ }^{146}$ T. Farooque, ${ }^{156}$

S. M. Farrington, ${ }^{16}$ P. Farthouat, ${ }^{29}$ P. Fassnacht, ${ }^{29}$ D. Fassouliotis, ${ }^{8}$ B. Fatholahzadeh, ${ }^{156}$ A. Favareto, ${ }^{87 a, 87 b}$ L. Fayard, ${ }^{113}$ S. Fazio, ${ }^{36 a, 36 b}$ R. Febbraro, ${ }^{33}$ P. Federic, ${ }^{142 a}$ O. L. Fedin, ${ }^{119}$ W. Fedorko, ${ }^{86}$ M. Fehling-Kaschek, ${ }^{47}$ L. Feligioni, ${ }^{81}$ D. Fellmann,,${ }^{5}$ C. Feng, ${ }^{32 \mathrm{~d}}$ E. J. Feng, ${ }^{30}$ A. B. Fenyuk, ${ }^{126}$ J. Ferencei, ${ }^{142 \mathrm{~b}}$ J. Ferland, ${ }^{91}$ W. Fernando, ${ }^{107}$ S. Ferrag, ${ }^{52}$ J. Ferrando, ${ }^{52}$ V. Ferrara, ${ }^{41}$ A. Ferrari, ${ }^{164}$ P. Ferrari, ${ }^{103}$ R. Ferrari, ${ }^{117 a}$ A. Ferrer,${ }^{165}$ M. L. Ferrer, ${ }^{46}$ D. Ferrere,${ }^{48}$ C. Ferretti ${ }^{85}$ A. Ferretto Parodi,${ }^{49 a}{ }^{49 b}$ M. Fiascaris, ${ }^{30}$ F. Fiedler ${ }^{79}$ A. Filipčič ${ }^{72}$ A. Filippas, ${ }^{9}$ F. Filthaut, ${ }^{102}$ M. Fincke-Keeler, ${ }^{167}$ M. C. N. Fiolhais, ${ }^{122 a, i}$ L. Fiorini, ${ }^{165}$ A. Firan, ${ }^{39}$ G. Fischer ${ }^{41}$ P. Fischer, ${ }^{20}$ M. J. Fisher, ${ }^{107}$ M. Flechl, ${ }^{47}$ I. Fleck, ${ }^{139}$ J. Fleckner, ${ }^{79}$ P. Fleischmann, ${ }^{171}$ S. Fleischmann, ${ }^{172}$ T. Flick, ${ }^{172}$ L. R. Flores Castillo, ${ }^{170}$ M. J. Flowerdew, ${ }^{97}$ M. Fokitis, ${ }^{9}$ T. Fonseca Martin, ${ }^{16}$ D. A. Forbush, ${ }^{136}$ A. Formica, ${ }^{134}$ A. Forti, ${ }^{80}$ D. Fortin, ${ }^{157 a}$ J. M. Foster, ${ }^{80}$ D. Fournier ${ }^{113}$ A. Foussat, ${ }^{29}$ A. J. Fowler,${ }^{44}$ K. Fowler ${ }^{135}$ H. Fox,${ }^{69}$ P. Francavilla, ${ }^{11}$ S. Franchino, ${ }^{117 a, 117 b}$ D. Francis, ${ }^{29}$ T. Frank,${ }^{169}$ M. Franklin, ${ }^{56}$ S. Franz, ${ }^{29}$ M. Fraternali, ${ }^{117 a, 117 b}$ S. Fratina, ${ }^{18}$ S. T. French, ${ }^{27}$ F. Friedrich, ${ }^{43}$ R. Froeschl,${ }^{29}$ D. Froidevaux, ${ }^{29}$ J. A. Frost,${ }^{27}$ C. Fukunaga, ${ }^{154}$ E. Fullana Torregrosa, ${ }^{29}$ J. Fuster, ${ }^{165}$ C. Gabaldon, ${ }^{29}$ O. Gabizon, ${ }^{169}$ T. Gadfort ${ }^{24}$ S. Gadomski, ${ }^{48}$ G. Gagliardi, ${ }^{49 a}, 49 \mathrm{~b}$ P. Gagnon, ${ }^{59}$ C. Galea,${ }^{96}$ E. J. Gallas, ${ }^{116}$ V. Gallo, ${ }^{16}$ B. J. Gallop, ${ }^{127}$ P. Gallus, ${ }^{123}$ K. K. Gan, ${ }^{107}$ Y. S. Gao, ${ }^{141, f}$ V. A. Gapienko, ${ }^{126}$ A. Gaponenko ${ }^{14}$ F. Garberson, ${ }^{173}$ M. Garcia-Sciveres, ${ }^{14}$ C. García, ${ }^{165}$ J. E. García Navarro, ${ }^{165}$ R. W. Gardner, ${ }^{30}$ N. Garelli, ${ }^{29}$ H. Garitaonandia, ${ }^{103}$ V. Garonne, ${ }^{29}$ J. Garvey, ${ }^{17}$ C. Gatti, ${ }^{46}$ G. Gaudio, ${ }^{117 a}$ O. Gaumer, ${ }^{48}$ B. Gaur, ${ }^{139}$ L. Gauthier, ${ }^{134}$ I. L. Gavrilenko, ${ }^{92}$ C. Gay, ${ }^{166}$ G. Gaycken, ${ }^{20}$ J-C. Gayde, ${ }^{29}$ E. N. Gazis, ${ }^{9}$ P. Ge ${ }^{32 \mathrm{~d}}$ C. N.P. Gee, ${ }^{127}$ D. A. A. Geerts, ${ }^{103}$ Ch. Geich-Gimbel,${ }^{20}$ K. Gellerstedt, ${ }^{144 a, 144 b}$ C. Gemme, ${ }^{49 a}$ A. Gemmell, ${ }^{52}$ M. H. Genest,${ }^{54}$ S. Gentile, ${ }^{130 a, 130 \mathrm{~b}}$ M. George,${ }^{53}$ S. George,${ }^{74}$ P. Gerlach, ${ }^{172}$ A. Gershon,,${ }^{151}$ C. Geweniger, ${ }^{57 \mathrm{a}}$ H. Ghazlane, ${ }^{133 \mathrm{~b}}$ N. Ghodbane, ${ }^{33}$ B. Giacobbe, ${ }^{19 \mathrm{a}} \mathrm{S}$. Giagu, ${ }^{130 \mathrm{a}, 130 \mathrm{~b}}$ V. Giakoumopoulou, ${ }^{8}$ 
V. Giangiobbe, ${ }^{11}$ F. Gianotti, ${ }^{29}$ B. Gibbard, ${ }^{24}$ A. Gibson, ${ }^{156}$ S. M. Gibson, ${ }^{29}$ L. M. Gilbert, ${ }^{116}$ V. Gilewsky, ${ }^{89}$ D. Gillberg, ${ }^{28}$ A. R. Gillman, ${ }^{127}$ D. M. Gingrich ${ }^{2, e}$ J. Ginzburg, ${ }^{151}$ N. Giokaris, ${ }^{8}$ M. P. Giordani, ${ }^{162 \mathrm{c}}$ R. Giordano, ${ }^{100 a, 100 b}$ F. M. Giorgi, ${ }^{15}$ P. Giovannini, ${ }^{97}$ P. F. Giraud, ${ }^{134}$ D. Giugni, ${ }^{87 a}$ M. Giunta, ${ }^{91}$ P. Giusti, ${ }^{19 a}$ B. K. Gjelsten, ${ }^{15}$ L. K. Gladilin, ${ }^{95}$ C. Glasman, ${ }^{78}$ J. Glatzer, ${ }^{47}$ A. Glazov, ${ }^{41}$ K. W. Glitza, ${ }^{172}$ G. L. Glonti, ${ }^{63}$ J. R. Goddard, ${ }^{73}$ J. Godfrey, ${ }^{140}$ J. Godlewski, ${ }^{29}$ M. Goebel, ${ }^{41}$ T. Göpfert, ${ }^{43}$ C. Goeringer, ${ }^{79}$ C. Gössling, ${ }^{42}$ T. Göttfert, ${ }^{97}$ S. Goldfarb, ${ }^{85}$ T. Golling, ${ }^{173}$ S. N. Golovnia, ${ }^{126}$ A. Gomes, ${ }^{122 a, c}$ L. S. Gomez Fajardo, ${ }^{41}$ R. Gonçalo, ${ }^{74}$ J. Goncalves Pinto Firmino Da Costa, ${ }^{41}$ L. Gonella, ${ }^{20}$ A. Gonidec, ${ }^{29}$ S. Gonzalez, ${ }^{170}$ S. González de la Hoz, ${ }^{165}$ G. Gonzalez Parra, ${ }^{11}$ M. L. Gonzalez Silva, ${ }^{26}$ S. Gonzalez-Sevilla, ${ }^{48}$ J. J. Goodson, ${ }^{146}$ L. Goossens, ${ }^{29}$

P. A. Gorbounov ${ }^{93}$ H. A. Gordon,${ }^{24}$ I. Gorelov, ${ }^{101}$ G. Gorfine, ${ }^{172}$ B. Gorini,${ }^{29}$ E. Gorini, ${ }^{70 a, 70 b}$ A. Gorišek, ${ }^{72}$ E. Gornicki, ${ }^{38}$ S. A. Gorokhov, ${ }^{126}$ V. N. Goryachev, ${ }^{126}$ B. Gosdzik, ${ }^{41}$ M. Gosselink, ${ }^{103}$ M. I. Gostkin, ${ }^{63}$ I. Gough Eschrich, ${ }^{161}$ M. Gouighri, ${ }^{133 a}$ D. Goujdami, ${ }^{133 c}$ M. P. Goulette, ${ }^{48}$ A. G. Goussiou, ${ }^{136}$ C. Goy, ${ }^{4}$ S. Gozpinar, ${ }^{22}$ I. Grabowska-Bold,${ }^{37}$ P. Grafström, ${ }^{29}$ K-J. Grahn, ${ }^{41}$ F. Grancagnolo ${ }^{70 a}$ S. Grancagnolo, ${ }^{15}$ V. Grassi, ${ }^{146}$ V. Gratchev, ${ }^{119}$ N. Grau, ${ }^{34}$ H. M. Gray, ${ }^{29}$ J. A. Gray, ${ }^{146}$ E. Graziani, ${ }^{132 a}$ O. G. Grebenyuk, ${ }^{119}$ T. Greenshaw, ${ }^{71}$ Z. D. Greenwood, ${ }^{24, m}$ K. Gregersen, ${ }^{35}$ I. M. Gregor, ${ }^{41}$ P. Grenier, ${ }^{141}$ J. Griffiths, ${ }^{136}$ N. Grigalashvili, ${ }^{63}$ A. A. Grillo, ${ }^{135}$ S. Grinstein, ${ }^{11}$ Y. V. Grishkevich, ${ }^{95}$ J.-F. Grivaz, ${ }^{113}$ M. Groh,${ }^{97}$ E. Gross,${ }^{169}$ J. Grosse-Knetter ${ }^{53}$ J. Groth-Jensen, ${ }^{169}$ K. Grybel, ${ }^{139}$ V. J. Guarino, ${ }^{5}$ D. Guest, ${ }^{173}$ C. Guicheney, ${ }^{33}$ A. Guida, ${ }^{70 a, 70 b}$ S. Guindon, ${ }^{53}$ H. Guler, ${ }^{83,0}$ J. Gunther, ${ }^{123}$ B. Guo, ${ }^{156}$ J. Guo, ${ }^{34}$ A. Gupta, ${ }^{30}$ Y. Gusakov, ${ }^{63}$ V. N. Gushchin, ${ }^{126}$ A. Gutierrez, ${ }^{91}$ P. Gutierrez, ${ }^{109}$ N. Guttman, ${ }^{151}$ O. Gutzwiller, ${ }^{170}$ C. Guyot, ${ }^{134}$ C. Gwenlan, ${ }^{116}$ C. B. Gwilliam, ${ }^{71}$ A. Haas,${ }^{141}$ S. Haas, ${ }^{29}$ C. Haber, ${ }^{14}$ H. K. Hadavand,,${ }^{39}$ D. R. Hadley, ${ }^{17}$ P. Haefner,${ }^{97}$ F. Hahn,${ }^{29}$ S. Haider,${ }^{29}$ Z. Hajduk, ${ }^{38}$ H. Hakobyan, ${ }^{174}$ D. Hall, ${ }^{116}$ J. Haller, ${ }^{53}$ K. Hamacher, ${ }^{172}$ P. Hamal, ${ }^{111}$ M. Hamer, ${ }^{53}$ A. Hamilton, ${ }^{143 b, p}$ S. Hamilton, ${ }^{159}$ H. Han, ${ }^{32 a}$ L. Han, ${ }^{32 b}$ K. Hanagaki, ${ }^{114}$ K. Hanawa, ${ }^{158}$ M. Hance,${ }^{14}$ C. Handel,${ }^{79}$ P. Hanke,${ }^{57 a}$ J. R. Hansen, ${ }^{35}$ J. B. Hansen, ${ }^{35}$ J. D. Hansen, ${ }^{35}$ P. H. Hansen, ${ }^{35}$ P. Hansson, ${ }^{141}$ K. Hara, ${ }^{158}$ G. A. Hare, ${ }^{135}$ T. Harenberg, ${ }^{172}$ S. Harkusha, ${ }^{88}$ D. Harper, ${ }^{85}$ R. D. Harrington, ${ }^{45}$ O. M. Harris, ${ }^{136}$ K. Harrison, ${ }^{17}$ J. Hartert, ${ }^{47}$ F. Hartjes, ${ }^{103}$ T. Haruyama, ${ }^{64}$ A. Harvey, ${ }^{55}$ S. Hasegawa, ${ }^{99}$ Y. Hasegawa, ${ }^{138}$ S. Hassani, ${ }^{134}$ M. Hatch, ${ }^{29}$ D. Hauff,${ }^{97}$ S. Haug, ${ }^{16}$ M. Hauschild ${ }^{29}$ R. Hauser, ${ }^{86}$ M. Havranek, ${ }^{20}$ B. M. Hawes, ${ }^{116}$ C. M. Hawkes, ${ }^{17}$ R. J. Hawkings, ${ }^{29}$ A. D. Hawkins, ${ }^{77}$ D. Hawkins, ${ }^{161}$ T. Hayakawa, ${ }^{65}$ T. Hayashi, ${ }^{158}$ D. Hayden ${ }^{74}$ H. S. Hayward,${ }^{71}$ S. J. Haywood, ${ }^{127}$ E. Hazen, ${ }^{21}$ M. He, ${ }^{32 \mathrm{~d}}$ S. J. Head,${ }^{17}$ V. Hedberg,${ }^{77}$ L. Heelan, ${ }^{7}$ S. Heim ${ }^{86}$ B. Heinemann, ${ }^{14}$ S. Heisterkamp ${ }^{35}$ L. Helary, ${ }^{4}$ C. Heller,${ }^{96}$ M. Heller ${ }^{29}$ S. Hellman, ${ }^{144 a, 144 b}$ D. Hellmich,${ }^{20}$ C. Helsens, ${ }^{11}$ R. C. W. Henderson, ${ }^{69}$ M. Henke, ${ }^{57 a}$ A. Henrichs, ${ }^{53}$ A. M. Henriques Correia, ${ }^{29}$ S. Henrot-Versille, ${ }^{113}$ F. Henry-Couannier, ${ }^{81}$ C. Hensel, ${ }^{53}$ T. Hen $\beta,{ }^{172}$ C. M. Hernandez,${ }^{7}$ Y. Hernández Jiménez, ${ }^{165}$ R. Herrberg, ${ }^{15}$ A. D. Hershenhorn, ${ }^{150}$ G. Herten, ${ }^{47}$ R. Hertenberger, ${ }^{96}$ L. Hervas, ${ }^{29}$ N. P. Hessey, ${ }^{103}$ E. Higón-Rodriguez, ${ }^{165}$ D. Hill, ${ }^{5, a}$ J. C. Hill, ${ }^{27}$ N. Hill, ${ }^{5}$ K. H. Hiller ${ }^{41}$ S. Hillert, ${ }^{20}$ S. J. Hillier, ${ }^{17}$ I. Hinchliffe, ${ }^{14}$ E. Hines, ${ }^{18}$ M. Hirose,${ }^{114}$ F. Hirsch, ${ }^{42}$ D. Hirschbuehl, ${ }^{172}$ J. Hobbs, ${ }^{146}$ N. Hod, ${ }^{151}$ M. C. Hodgkinson, ${ }^{137}$ P. Hodgson, ${ }^{137}$ A. Hoecker, ${ }^{29}$ M. R. Hoeferkamp, ${ }^{101}$ J. Hoffman,${ }^{39}$ D. Hoffmann, ${ }^{81}$ M. Hohlfeld, ${ }^{79}$ M. Holder, ${ }^{139}$ S. O. Holmgren, ${ }^{144 a}$ T. Holy, ${ }^{125}$ J. L. Holzbauer,${ }^{86}$ Y. Homma, ${ }^{65}$ T. M. Hong, ${ }^{118}$ L. Hooft van Huysduynen, ${ }^{106}$ T. Horazdovsky, ${ }^{125}$ C. Horn, ${ }^{141}$ S. Horner, ${ }^{47}$ J-Y. Hostachy, ${ }^{54}$ S. Hou, ${ }^{149}$ M. A. Houlden, ${ }^{71}$ A. Hoummada, ${ }^{133 a}$ J. Howarth, ${ }^{80}$ D. F. Howell, ${ }^{116}$ I. Hristova, ${ }^{15}$ J. Hrivnac, ${ }^{113}$ I. Hruska, ${ }^{123}$ T. Hryn'ova, ${ }^{4}$ P. J. Hsu, ${ }^{79}$ S.-C. Hsu, ${ }^{14}$ G. S. Huang,,${ }^{109}$ Z. Hubacek, ${ }^{125}$ F. Hubaut,${ }^{81}$ F. Huegging, ${ }^{20}$ A. Huettmann, ${ }^{41}$ T. B. Huffman, ${ }^{116}$ E. W. Hughes,${ }^{34}$ G. Hughes,${ }^{69}$ R. E. Hughes-Jones, ${ }^{80}$ M. Huhtinen, ${ }^{29}$ P. Hurst,${ }^{56}$ M. Hurwitz, ${ }^{14}$

U. Husemann, ${ }^{41}$ N. Huseynov, ${ }^{63, q}$ J. Huston, ${ }^{86}$ J. Huth, ${ }^{56}$ G. Iacobucci, ${ }^{48}$ G. Iakovidis, ${ }^{9}$ M. Ibbotson, ${ }^{80}$

I. Ibragimov, ${ }^{139} \mathrm{R}$. Ichimiya, ${ }^{65} \mathrm{~L}$. Iconomidou-Fayard, ${ }^{113} \mathrm{~J}$. Idarraga, ${ }^{113} \mathrm{P}$. Iengo, ${ }^{100 a}$ O. Igonkina, ${ }^{103} \mathrm{Y}$. Ikegami, ${ }^{64}$ M. Ikeno, ${ }^{64}$ Y. Ilchenko, ${ }^{39}$ D. Iliadis, ${ }^{152}$ N. Ilic, ${ }^{156}$ D. Imbault,${ }^{76}$ M. Imori, ${ }^{153}$ T. Ince, ${ }^{20}$ J. Inigo-Golfin, ${ }^{29}$ P. Ioannou, ${ }^{8}$ M. Iodice, ${ }^{132 a}$ V. Ippolito, ${ }^{130 a, 130 b}$ A. Irles Quiles, ${ }^{165} \mathrm{C}$. Isaksson, ${ }^{164}$ A. Ishikawa, ${ }^{65} \mathrm{M}$. Ishino, ${ }^{66}$

R. Ishmukhametov, ${ }^{39} \mathrm{C}$. Issever, ${ }^{116} \mathrm{~S}$. Istin, ${ }^{18 \mathrm{a}} \mathrm{A}$. V. Ivashin, ${ }^{126} \mathrm{~W}$. Iwanski, ${ }^{38} \mathrm{H}$. Iwasaki, ${ }^{64} \mathrm{~J}$. M. Izen, ${ }^{40} \mathrm{~V}$. Izzo, ${ }^{100 a}$ B. Jackson, ${ }^{118}$ J. N. Jackson, ${ }^{71}$ P. Jackson, ${ }^{141}$ M. R. Jaekel, ${ }^{29}$ V. Jain, ${ }^{59}$ K. Jakobs, ${ }^{47}$ S. Jakobsen, ${ }^{35}$ J. Jakubek,${ }^{125}$

D. K. Jana, ${ }^{109}$ E. Jankowski, ${ }^{156}$ E. Jansen, ${ }^{75}$ H. Jansen, ${ }^{29}$ A. Jantsch,${ }^{97}$ M. Janus, ${ }^{20}$ G. Jarlskog, ${ }^{77}$ L. Jeanty, ${ }^{56}$

K. Jelen, ${ }^{37}$ I. Jen-La Plante, ${ }^{30}$ P. Jenni, ${ }^{29}$ A. Jeremie, ${ }^{4}$ P. Jež ${ }^{35}$ S. Jézéquel,${ }^{4}$ M. K. Jha, ${ }^{19 a}$ H. Ji, ${ }^{170}$ W. Ji,${ }^{79}$ J. Jia, ${ }^{146}$ Y. Jiang, ${ }^{32 b}$ M. Jimenez Belenguer, ${ }^{41}$ G. Jin, ${ }^{32 b}$ S. Jin, ${ }^{32 a}$ O. Jinnouchi, ${ }^{155}$ M. D. Joergensen, ${ }^{35}$ D. Joffe, ${ }^{39}$ L. G. Johansen, ${ }^{13}$ M. Johansen, ${ }^{144 a, 144 b}$ K. E. Johansson, ${ }^{144 a}$ P. Johansson, ${ }^{137}$ S. Johnert, ${ }^{41}$ K. A. Johns, ${ }^{6}$ K. Jon-And, ${ }^{144 a, 144 b}$ G. Jones, ${ }^{80}$ R. W. L. Jones, ${ }^{69}$ T. W. Jones, ${ }^{75}$ T. J. Jones,${ }^{71}$ O. Jonsson, ${ }^{29}$ C. Joram, ${ }^{29}$ P. M. Jorge, ${ }^{122 \mathrm{a}}$ J. Joseph, ${ }^{14}$ T. Jovin, ${ }^{12 \mathrm{~b}}$ X. Ju, ${ }^{170}$ C. A. Jung,${ }^{42}$ R. M. Jungst,${ }^{29}$ V. Juranek, ${ }^{123}$ P. Jussel,${ }^{60}$ A. Juste Rozas, ${ }^{11}$ V. V. Kabachenko, ${ }^{126}$ S. Kabana, ${ }^{16}$ M. Kaci, ${ }^{165}$ A. Kaczmarska, ${ }^{38}$ P. Kadlecik, ${ }^{35}$ M. Kado, ${ }^{113}$ 
H. Kagan, ${ }^{107}$ M. Kagan,${ }^{56}$ S. Kaiser, ${ }^{97}$ E. Kajomovitz, ${ }^{150}$ S. Kalinin, ${ }^{172}$ L. V. Kalinovskaya, ${ }^{63}$ S. Kama, ${ }^{39}$ N. Kanaya, ${ }^{153}$ M. Kaneda, ${ }^{29}$ S. Kaneti, ${ }^{27}$ T. Kanno, ${ }^{155}$ V. A. Kantserov, ${ }^{94}$ J. Kanzaki, ${ }^{64}$ B. Kaplan, ${ }^{173}$ A. Kapliy, ${ }^{30}$ J. Kaplon, ${ }^{29}$ D. Kar, ${ }^{43}$ M. Karagounis,${ }^{20}$ M. Karagoz, ${ }^{116}$ M. Karnevskiy, ${ }^{41}$ K. Karr, ${ }^{5}$ V. Kartvelishvili, ${ }^{69}$ A. N. Karyukhin, ${ }^{126}$ L. Kashif, ${ }^{170}$ G. Kasieczka, ${ }^{57 b}$ R. D. Kass, ${ }^{107}$ A. Kastanas, ${ }^{13}$ M. Kataoka, ${ }^{4}$ Y. Kataoka, ${ }^{153}$

E. Katsoufis, ${ }^{9}$ J. Katzy, ${ }^{41}$ V. Kaushik,${ }^{6}$ K. Kawagoe, ${ }^{65}$ T. Kawamoto, ${ }^{153}$ G. Kawamura, ${ }^{79}$ M. S. Kayl, ${ }^{103}$ V. A. Kazanin, ${ }^{105}$ M. Y. Kazarinov, ${ }^{63}$ R. Keeler, ${ }^{167}$ R. Kehoe, ${ }^{39}$ M. Keil,${ }^{53}$ G. D. Kekelidze ${ }^{63}$ J. Kennedy, ${ }^{96}$ C. J. Kenney, ${ }^{141}$ M. Kenyon, ${ }^{52}$ O. Kepka, ${ }^{123}$ N. Kerschen, ${ }^{29}$ B. P. Kerševan, ${ }^{72}$ S. Kersten, ${ }^{172}$ K. Kessoku, ${ }^{153}$ J. Keung, ${ }^{156}$ F. Khalil-zada, ${ }^{10}$ H. Khandanyan, ${ }^{163}$ A. Khanov, ${ }^{110}$ D. Kharchenko, ${ }^{63}$ A. Khodinov, ${ }^{94}$ A. G. Kholodenko, ${ }^{126}$ A. Khomich, ${ }^{57 a}$ T. J. Khoo, ${ }^{27}$ G. Khoriauli, ${ }^{20}$ A. Khoroshilov, ${ }^{172}$ N. Khovanskiy, ${ }^{63}$ V. Khovanskiy, ${ }^{93}$ E. Khramov, ${ }^{63}$ J. Khubua, ${ }^{50 \mathrm{~b}}$ H. Kim, ${ }^{144 \mathrm{a}, 144 \mathrm{~b}}$ M. S. Kim, ${ }^{2}$ P. C. Kim, ${ }^{141}$ S. H. Kim, ${ }^{158}$ N. Kimura, ${ }^{168}$ O. Kind,,${ }^{15}$ B. T. King, ${ }^{71}$ M. King, ${ }^{65}$ R. S. B. King,,${ }^{116}$ J. Kirk, ${ }^{127}$ L. E. Kirsch, ${ }^{22}$ A. E. Kiryunin,${ }^{97}$ T. Kishimoto, ${ }^{65}$ D. Kisielewska, ${ }^{37}$ T. Kittelmann, ${ }^{121}$ A. M. Kiver, ${ }^{126}$ E. Kladiva, ${ }^{142 b}$ J. Klaiber-Lodewigs ${ }^{42}$ M. Klein, ${ }^{71}$ U. Klein, ${ }^{71}$ K. Kleinknecht, ${ }^{79}$ M. Klemetti, ${ }^{83}$ A. Klier, ${ }^{169}$ P. Klimek, ${ }^{144 a, 144 b}$ A. Klimentov, ${ }^{24}$ R. Klingenberg, ${ }^{42}$ E. B. Klinkby, ${ }^{35}$ T. Klioutchnikova, ${ }^{29}$ P. F. Klok, ${ }^{102}$ S. Klous, ${ }^{103}$ E.-E. Kluge,${ }^{57 a}$ T. Kluge, ${ }^{71}$ P. Kluit, ${ }^{103}$ S. Kluth, ${ }^{97}$ N. S. Knecht, ${ }^{156}$ E. Kneringer, ${ }^{60}$ J. Knobloch, ${ }^{29}$ E. B. F. G. Knoops, ${ }^{81}$ A. Knue, ${ }^{53}$ B. R. Ko, ${ }^{44}$ T. Kobayashi ${ }^{153}$ M. Kobel, ${ }^{43}$ M. Kocian, ${ }^{141}$ P. Kodys,${ }^{124}$ K. Köneke, ${ }^{29}$ A. C. König, ${ }^{102}$ S. Koenig, ${ }^{79}$ L. Köpke, ${ }^{79}$ F. Koetsveld, ${ }^{102}$ P. Koevesarki, ${ }^{20}$ T. Koffas, ${ }^{28}$ E. Koffeman, ${ }^{103}$ L. A. Kogan, ${ }^{116}$ F. Kohn, ${ }^{53}$ Z. Kohout, ${ }^{125}$ T. Kohriki, ${ }^{64}$ T. Koi, ${ }^{141}$ T. Kokott, ${ }^{20}$ G. M. Kolachev, ${ }^{105}$ H. Kolanoski, ${ }^{15}$ V. Kolesnikov, ${ }^{63}$ I. Koletsou, ${ }^{87 a}$ J. Koll, ${ }^{86}$ D. Kollar, ${ }^{29}$ M. Kollefrath, ${ }^{47}$ S. D. Kolya ${ }^{80}$ A. A. Komar, ${ }^{92}$ Y. Komori, ${ }^{153}$ T. Kondo,${ }^{64}$ T. Kono, ${ }^{41, r}$ A. I. Kononov, ${ }^{47}$ R. Konoplich, ${ }^{106, s}$ N. Konstantinidis, ${ }^{75}$ A. Kootz, ${ }^{172}$ S. Koperny, ${ }^{37}$ K. Korcyl, ${ }^{38}$ K. Kordas, ${ }^{152}$ V. Koreshev, ${ }^{126}$

A. Korn, ${ }^{116}$ A. Korol, ${ }^{105}$ I. Korolkov, ${ }^{11}$ E. V. Korolkova, ${ }^{137}$ V. A. Korotkov, ${ }^{126}$ O. Kortner, ${ }^{97}$ S. Kortner, ${ }^{97}$ V. V. Kostyukhin, ${ }^{20}$ M. J. Kotamäki, ${ }^{29}$ S. Kotov, ${ }^{97}$ V. M. Kotov, ${ }^{63}$ A. Kotwal, ${ }^{44}$ C. Kourkoumelis, ${ }^{8}$ V. Kouskoura, ${ }^{152}$ A. Koutsman, ${ }^{157 a}$ R. Kowalewski, ${ }^{167}$ T.Z. Kowalski, ${ }^{37}$ W. Kozanecki, ${ }^{134}$ A. S. Kozhin, ${ }^{126}$ V. Kral, ${ }^{125}$ V. A. Kramarenko, ${ }^{95}$ G. Kramberger, ${ }^{72}$ M. W. Krasny, ${ }^{76}$ A. Krasznahorkay, ${ }^{106}$ J. Kraus, ${ }^{86}$ J. K. Kraus,${ }^{20}$ A. Kreisel, ${ }^{151}$ F. Krejci, ${ }^{125}$ J. Kretzschmar, ${ }^{71}$ N. Krieger, ${ }^{53}$ P. Krieger, ${ }^{156}$ K. Kroeninger, ${ }^{53}$ H. Kroha, ${ }^{97}$ J. Kroll, ${ }^{118}$ J. Kroseberg, ${ }^{20}$ J. Krstic, ${ }^{12 a}$ U. Kruchonak ${ }^{63}$ H. Krüger, ${ }^{20}$ T. Kruker, ${ }^{16}$ N. Krumnack, ${ }^{62}$ Z. V. Krumshteyn, ${ }^{63}$ A. Kruth ${ }^{20}$ T. Kubota, ${ }^{84}$ S. Kuehn, ${ }^{47}$ A. Kugel, ${ }^{57 \mathrm{c}}$ T. Kuhl, ${ }^{41}$ D. Kuhn, ${ }^{60}$ V. Kukhtin, ${ }^{63}$ Y. Kulchitsky, ${ }^{88}$ S. Kuleshov,${ }^{31 b}$ C. Kummer, ${ }^{96}$ M. Kuna, ${ }^{76}$ N. Kundu, ${ }^{116}$ J. Kunkle, ${ }^{118}$ A. Kupco, ${ }^{123}$ H. Kurashige,${ }^{65}$ M. Kurata, ${ }^{158}$ Y. A. Kurochkin,${ }^{88}$ V. Kus, ${ }^{123}$ E. S. Kuwertz, ${ }^{145}$ M. Kuze, ${ }^{155}$ J. Kvita, ${ }^{140}$ R. Kwee, ${ }^{15}$ A. La Rosa,${ }^{48}$ L. La Rotonda, ${ }^{36 a, 36 b}$ L. Labarga ${ }^{78}$ J. Labbe, ${ }^{4}$

S. Lablak, ${ }^{133 a}$ C. Lacasta, ${ }^{165}$ F. Lacava, ${ }^{130 a, 130 b}$ H. Lacker,${ }^{15}$ D. Lacour,${ }^{76}$ V. R. Lacuesta, ${ }^{165}$ E. Ladygin, ${ }^{63}$

R. Lafaye, ${ }^{4}$ B. Laforge, ${ }^{76}$ T. Lagouri, ${ }^{78}$ S. Lai, ${ }^{47}$ E. Laisne,${ }^{54}$ M. Lamanna, ${ }^{29}$ C. L. Lampen, ${ }^{6}$ W. Lampl,${ }^{6}$ E. Lancon, ${ }^{134}$ U. Landgraf, ${ }^{47}$ M. P. J. Landon, ${ }^{73}$ H. Landsman, ${ }^{150}$ J. L. Lane, ${ }^{80}$ C. Lange, ${ }^{41}$ A. J. Lankford, ${ }^{161}$ F. Lanni, ${ }^{24}$ K. Lantzsch, ${ }^{172}$ S. Laplace, ${ }^{76}$ C. Lapoire, ${ }^{20}$ J. F. Laporte, ${ }^{134}$ T. Lari, ${ }^{87 a}$ A. V. Larionov, ${ }^{126}$ A. Larner, ${ }^{116}$ C. Lasseur, ${ }^{29}$ M. Lassnig, ${ }^{29}$ P. Laurelli, ${ }^{46}$ W. Lavrijsen, ${ }^{14}$ P. Laycock, ${ }^{71}$ A. B. Lazarev, ${ }^{63}$ O. Le Dortz, ${ }^{76}$

E. Le Guirriec, ${ }^{81}$ C. Le Maner, ${ }^{156}$ E. Le Menedeu, ${ }^{9}$ C. Lebel,${ }^{91}$ T. LeCompte, ${ }^{5}$ F. Ledroit-Guillon,${ }^{54}$ H. Lee, ${ }^{103}$ J. S. H. Lee, ${ }^{114}$ S. C. Lee, ${ }^{149}$ L. Lee, ${ }^{173}$ M. Lefebvre, ${ }^{167}$ M. Legendre, ${ }^{134}$ A. Leger, ${ }^{48}$ B. C. LeGeyt, ${ }^{118}$ F. Legger, ${ }^{96}$ C. Leggett, ${ }^{14}$ M. Lehmacher, ${ }^{20}$ G. Lehmann Miotto, ${ }^{29}$ X. Lei, ${ }^{6}$ M. A. L. Leite, ${ }^{23 d}$ R. Leitner, ${ }^{124}$ D. Lellouch, ${ }^{169}$ M. Leltchouk, ${ }^{34}$ B. Lemmer, ${ }^{53}$ V. Lendermann, ${ }^{57 a}$ K. J. C. Leney, ${ }^{143 b}$ T. Lenz, ${ }^{103}$ G. Lenzen, ${ }^{172}$ B. Lenzi, ${ }^{29}$

K. Leonhardt ${ }^{43}$ S. Leontsinis, ${ }^{9}$ C. Leroy, ${ }^{91}$ J-R. Lessard, ${ }^{167}$ J. Lesser,${ }^{144 a}$ C. G. Lester ${ }^{27}$ A. Leung Fook Cheong, ${ }^{170}$ J. Levêque, ${ }^{4}$ D. Levin, ${ }^{85}$ L. J. Levinson, ${ }^{169}$ M. S. Levitski, ${ }^{126}$ A. Lewis, ${ }^{16}$ G. H. Lewis, ${ }^{106}$ A. M. Leyko, ${ }^{20}$ M. Leyton, ${ }^{15}$ B. Li ${ }^{81}$ H. Li $,{ }^{170, t} \mathrm{~S} . \mathrm{Li},{ }^{32 b, \mathrm{u}}$ X. Li ${ }^{85}$ Z. Liang,${ }^{16, v}$ H. Liao,${ }^{33}$ B. Liberti, ${ }^{131 \mathrm{a}}$ P. Lichard ${ }^{29}$ M. Lichtnecker,${ }^{96}$ K. Lie, ${ }^{163}$ W. Liebig, ${ }^{13}$ R. Lifshitz ${ }^{150}$ C. Limbach, ${ }^{20}$ A. Limosani, ${ }^{84}$ M. Limper, ${ }^{61}$ S. C. Lin, ${ }^{149, w}$ F. Linde, ${ }^{103}$ J. T. Linnemann, ${ }^{86}$ E. Lipeles, ${ }^{18}$ L. Lipinsky, ${ }^{123}$ A. Lipniacka,${ }^{13}$ T. M. Liss, ${ }^{163}$ D. Lissauer,${ }^{24}$ A. Lister,${ }^{48}$ A. M. Litke, ${ }^{135}$ C. Liu, ${ }^{28}$ D. Liu, ${ }^{149}$ H. Liu, ${ }^{85}$ J. B. Liu,${ }^{85}$ M. Liu, ${ }^{32 b}$ S. Liu, ${ }^{2}$ Y. Liu, ${ }^{32 b}$ M. Livan,,${ }^{117 a, 117 b}$ S. S. A. Livermore, ${ }^{116}$ A. Lleres,${ }^{54}$ J. Llorente Merino, ${ }^{78}$ S. L. Lloyd, ${ }^{73}$ E. Lobodzinska, ${ }^{41}$ P. Loch,${ }^{6}$ W. S. Lockman, ${ }^{135}$ T. Loddenkoetter ${ }^{20}$ F. K. Loebinger, ${ }^{80}$ A. Loginov, ${ }^{173}$ C. W. Loh, ${ }^{166}$ T. Lohse, ${ }^{15}$ K. Lohwasser ${ }^{47}$ M. Lokajicek, ${ }^{123}$ J. Loken, ${ }^{116}$ V. P. Lombardo,${ }^{4}$ R. E. Long, ${ }^{69}$ L. Lopes, ${ }^{122 a, c}$ D. Lopez Mateos,${ }^{56}$ J. Lorenz, ${ }^{96}$ M. Losada, ${ }^{160}$ P. Loscutoff, ${ }^{14}$ F. Lo Sterzo,${ }^{130 a, 130 b}$ M. J. Losty, ${ }^{157 a}$ X. Lou ${ }^{40}$ A. Lounis, ${ }^{113}$ K. F. Loureiro, ${ }^{160}$ J. Love, ${ }^{21}$ P. A. Love, ${ }^{69}$ A. J. Lowe, ${ }^{141, f}$ F. Lu, ${ }^{32 a}$ H. J. Lubatti, ${ }^{136}$ C. Luci, ${ }^{130 a, 130 b}$ A. Lucotte ${ }^{54}$ A. Ludwig, ${ }^{43}$ D. Ludwig, ${ }^{41}$ I. Ludwig, ${ }^{47}$ J. Ludwig, ${ }^{47}$ F. Luehring, ${ }^{59}$ G. Luijckx, ${ }^{103}$ D. Lumb,${ }^{47}$ L. Luminari, ${ }^{130 a}$ E. Lund, ${ }^{115}$ B. Lund-Jensen, ${ }^{145}$ B. Lundberg, ${ }^{77}$ J. Lundberg, ${ }^{144 a, 144 b}$ J. Lundquist, ${ }^{35}$ M. Lungwitz ${ }^{79}$ G. Lutz, ${ }^{97}$ D. Lynn, ${ }^{24}$ 
J. Lys, ${ }^{14}$ E. Lytken, ${ }^{77}$ H. Ma, ${ }^{24}$ L. L. Ma ${ }^{170}$ J. A. Macana Goia,${ }^{91}$ G. Maccarrone, ${ }^{46}$ A. Macchiolo, ${ }^{97}$ B. Maček, ${ }^{72}$ J. Machado Miguens, ${ }^{122 \mathrm{a}}$ R. Mackeprang, ${ }^{35}$ R. J. Madaras, ${ }^{14}$ W. F. Mader, ${ }^{43}$ R. Maenner, ${ }^{57 \mathrm{c}}$ T. Maeno, ${ }^{24}$ P. Mättig, ${ }^{172}$ S. Mättig, ${ }^{41}$ L. Magnoni, ${ }^{29}$ E. Magradze,${ }^{53}$ Y. Mahalalel,,${ }^{151}$ K. Mahboubi,${ }^{47}$ G. Mahout, ${ }^{17}$ C. Maiani, ${ }^{130 a, 130 b}$ C. Maidantchik, ${ }^{23 a}$ A. Maio, ${ }^{122 a, c}$ S. Majewski, ${ }^{24}$ Y. Makida,${ }^{64}$ N. Makovec, ${ }^{113}$ P. Mal, ${ }^{134}$ B. Malaescu, ${ }^{29}$ Pa. Malecki, ${ }^{38}$ P. Malecki, ${ }^{38}$ V. P. Maleev, ${ }^{119}$ F. Malek, ${ }^{54}$ U. Mallik, ${ }^{61}$ D. Malon,${ }^{5}$ C. Malone,${ }^{141}$ S. Maltezos,${ }^{9}$ V. Malyshev, ${ }^{105}$ S. Malyukov, ${ }^{29}$ R. Mameghani, ${ }^{96}$ J. Mamuzic, ${ }^{12 b}$ A. Manabe, ${ }^{64}$ L. Mandelli, ${ }^{87 a}$ I. Mandić, ${ }^{72}$ R. Mandrysch, ${ }^{15}$ J. Maneira, ${ }^{122 a}$ P. S. Mangeard ${ }^{86}$ L. Manhaes de Andrade Filho, ${ }^{23 a}$ I. D. Manjavidze, ${ }^{63}$ A. Mann, ${ }^{53}$ P. M. Manning, ${ }^{135}$ A. Manousakis-Katsikakis, ${ }^{8}$ B. Mansoulie, ${ }^{134}$ A. Manz, ${ }^{97}$ A. Mapelli, ${ }^{29}$ L. Mapelli, ${ }^{29}$ L. March, ${ }^{78}$ J.F. Marchand, ${ }^{28}$ F. Marchese, ${ }^{131 \mathrm{a}, 131 \mathrm{~b}}$ G. Marchiori, ${ }^{76}$ M. Marcisovsky, ${ }^{123}$ A. Marin, ${ }^{21, \mathrm{a}}$ C. P. Marino, ${ }^{167}$ F. Marroquim, ${ }^{23 a}$ R. Marshall, ${ }^{80}$ Z. Marshall, ${ }^{29}$ F. K. Martens, ${ }^{156}$ S. Marti-Garcia, ${ }^{165}$ A. J. Martin, ${ }^{173}$ B. Martin ${ }^{29}$ B. Martin, ${ }^{86}$ F. F. Martin, ${ }^{118}$ J. P. Martin, ${ }^{91}$ Ph. Martin, ${ }^{54}$ T. A. Martin, ${ }^{17}$ V. J. Martin, ${ }^{45}$ B. Martin dit Latour, ${ }^{48}$ S. Martin-Haugh ${ }^{147}$ M. Martinez,${ }^{11}$ V. Martinez Outschoorn,${ }^{56}$ A. C. Martyniuk, ${ }^{167}$ M. Marx ${ }^{80}$ F. Marzano, ${ }^{130 a}$ A. Marzin, ${ }^{109}$ L. Masetti, ${ }^{79}$ T. Mashimo, ${ }^{153}$ R. Mashinistov, ${ }^{92}$ J. Masik, ${ }^{80}$ A. L. Maslennikov, ${ }^{105}$ I. Massa, ${ }^{19 a, 19 b}$ G. Massaro, ${ }^{103}$ N. Massol, ${ }^{4}$ P. Mastrandrea, ${ }^{130 a, 130 b}$ A. Mastroberardino, ${ }^{36 a, 36 b}$ T. Masubuchi, ${ }^{153}$ M. Mathes,${ }^{20}$

P. Matricon, ${ }^{113}$ H. Matsumoto, ${ }^{153}$ H. Matsunaga ${ }^{153}$ T. Matsushita, ${ }^{65}$ C. Mattravers,${ }^{16, d}$ J. M. Maugain, ${ }^{29}$

J. Maurer, ${ }^{81}$ S. J. Maxfield, ${ }^{71}$ D. A. Maximov, ${ }^{105, g}$ E. N. May, ${ }^{5}$ A. Mayne, ${ }^{137}$ R. Mazini, ${ }^{149}$ M. Mazur, ${ }^{20}$

M. Mazzanti, ${ }^{87 a}$ E. Mazzoni, ${ }^{120 a, 120 b}$ S. P. Mc Kee, ${ }^{85}$ A. McCarn, ${ }^{163}$ R. L. McCarthy, ${ }^{146}$ T. G. McCarthy, ${ }^{28}$ N. A. McCubbin, ${ }^{127}$ K. W. McFarlane, ${ }^{55}$ J. A. Mcfayden, ${ }^{137}$ H. McGlone, ${ }^{52}$ G. Mchedlidze ${ }^{50 b}$ R. A. McLaren, ${ }^{29}$

T. Mclaughlan, ${ }^{17}$ S. J. McMahon, ${ }^{127}$ R. A. McPherson, ${ }^{167, k}$ A. Meade, ${ }^{82}$ J. Mechnich, ${ }^{103}$ M. Mechtel,${ }^{172}$

M. Medinnis, ${ }^{41}$ R. Meera-Lebbai, ${ }^{109}$ T. Meguro, ${ }^{114}$ R. Mehdiyev,${ }^{91}$ S. Mehlhase, ${ }^{35}$ A. Mehta, ${ }^{71}$ K. Meier,${ }^{57 a}$ B. Meirose ${ }^{77}$ C. Melachrinos,${ }^{30}$ B. R. Mellado Garcia, ${ }^{170}$ L. Mendoza Navas, ${ }^{160}$ Z. Meng, ${ }^{149, t}$ A. Mengarelli, ${ }^{19 a, 19 b}$ S. Menke, ${ }^{97}$ C. Menot ${ }^{29}$ E. Meoni,${ }^{11}$ K. M. Mercurio,${ }^{56}$ P. Mermod, ${ }^{48}$ L. Merola, ${ }^{100 a, 100 b}$ C. Meroni, ${ }^{87 a}$ F. S. Merritt, ${ }^{30}$ A. Messina, ${ }^{29}$ J. Metcalfe, ${ }^{101}$ A. S. Mete, ${ }^{62}$ C. Meyer, ${ }^{79}$ C. Meyer, ${ }^{30}$ J-P. Meyer, ${ }^{134}$ J. Meyer, ${ }^{171}$ J. Meyer ${ }^{53}$ T. C. Meyer, ${ }^{29}$ W. T. Meyer, ${ }^{62}$ J. Miao, ${ }^{32 d}$ S. Michal,${ }^{29}$ L. Micu ${ }^{25 a}$ R. P. Middleton, ${ }^{127}$ S. Migas, ${ }^{71}$ L. Mijović, ${ }^{41}$ G. Mikenberg, ${ }^{169}$ M. Mikestikova, ${ }^{123}$ M. Mikuž, ${ }^{72}$ D. W. Miller, ${ }^{30}$ R. J. Miller,${ }^{86}$ W. J. Mills, ${ }^{166}$ C. Mills, ${ }^{56}$ A. Milov, ${ }^{169}$ D. A. Milstead, ${ }^{144 a, 144 b}$ D. Milstein, ${ }^{169}$ A. A. Minaenko, ${ }^{126}$ M. Miñano Moya, ${ }^{165}$ I. A. Minashvili, ${ }^{63}$ A. I. Mincer, ${ }^{106}$ B. Mindur, ${ }^{37}$ M. Mineev, ${ }^{63}$ Y. Ming, ${ }^{170}$ L. M. Mir, ${ }^{11}$ G. Mirabelli, ${ }^{130 a}$ L. Miralles Verge, ${ }^{11}$ A. Misiejuk, ${ }^{74}$ J. Mitrevski, ${ }^{135}$ G. Y. Mitrofanov, ${ }^{126}$ V. A. Mitsou, ${ }^{165}$ S. Mitsui, ${ }^{64}$ P. S. Miyagawa, ${ }^{137}$ K. Miyazaki, ${ }^{65}$ J. U. Mjörnmark, ${ }^{77}$ T. Moa, ${ }^{144 a, 144 b}$ P. Mockett, ${ }^{136}$ S. Moed,${ }^{56}$ V. Moeller, ${ }^{27}$ K. Mönig, ${ }^{41}$ N. Möser, ${ }^{20}$ S. Mohapatra, ${ }^{146}$ W. Mohr,${ }^{47}$ S. Mohrdieck-Möck,${ }^{97}$ A. M. Moisseev, ${ }^{126, a}$ R. Moles-Valls, ${ }^{165}$ J. Molina-Perez, ${ }^{29}$ J. Monk, ${ }^{75}$ E. Monnier,${ }^{81}$ S. Montesano, ${ }^{87 a, 87 b}$ F. Monticelli, ${ }^{68}$ S. Monzani, ${ }^{19 a, 19 b}$ R. W. Moore, ${ }^{2}$ G. F. Moorhead ${ }^{84}$ C. Mora Herrera, ${ }^{48}$ A. Moraes,${ }^{52}$ N. Morange, ${ }^{134}$ J. Morel, ${ }^{53}$ G. Morello, ${ }^{36 a, 36 b}$ D. Moreno, ${ }^{79}$ M. Moreno Llácer, ${ }^{165}$ P. Morettini, ${ }^{49 a}$ M. Morii, ${ }^{56}$ J. Morin, ${ }^{73}$ A. K. Morley, ${ }^{29}$ G. Mornacchi, ${ }^{29}$ S. V. Morozov, ${ }^{94}$ J. D. Morris, ${ }^{73}$ L. Morvaj, ${ }^{99}$ H. G. Moser, ${ }^{97}$ M. Mosidze,${ }^{50 b}$ J. Moss, ${ }^{107}$ R. Mount, ${ }^{141}$ E. Mountricha, ${ }^{9, x}$ S. V. Mouraviev, ${ }^{92}$ E. J. W. Moyse, ${ }^{82}$ M. Mudrinic, ${ }^{12 b}$ F. Mueller, ${ }^{57 a}$ J. Mueller, ${ }^{121}$ K. Mueller, ${ }^{20}$ T. A. Müller, ${ }^{96}$ T. Mueller, ${ }^{79}$ D. Muenstermann, ${ }^{29}$ A. Muir, ${ }^{166}$ Y. Munwes, ${ }^{151}$ W. J. Murray, ${ }^{127}$ I. Mussche, ${ }^{103}$ E. Musto, ${ }^{100 a, 100 b}$ A. G. Myagkov, ${ }^{126}$ M. Myska, ${ }^{123}$ J. Nadal, ${ }^{11}$ K. Nagai, ${ }^{158}$ K. Nagano, ${ }^{64}$ Y. Nagasaka, ${ }^{58}$ M. Nagel, ${ }^{97}$ A. M. Nairz, ${ }^{29}$ Y. Nakahama, ${ }^{29}$ K. Nakamura, ${ }^{153}$ T. Nakamura, ${ }^{153}$ I. Nakano, ${ }^{108}$ G. Nanava, ${ }^{20}$ A. Napier, ${ }^{159}$ R. Narayan, ${ }^{57 b}$ M. Nash, ${ }^{75, d}$ N. R. Nation,${ }^{21}$ T. Nattermann, ${ }^{20}$ T. Naumann, ${ }^{41}$ G. Navarro, ${ }^{160}$ H. A. Neal, ${ }^{85}$ E. Nebot,${ }^{78}$ P. Yu. Nechaeva, ${ }^{92}$ A. Negri, ${ }^{117 a, 117 b}$ G. Negri, ${ }^{29}$ S. Nektarijevic, ${ }^{48}$ A. Nelson, ${ }^{161}$ S. Nelson, ${ }^{141}$ T. K. Nelson, ${ }^{141}$ S. Nemecek, ${ }^{123}$ P. Nemethy, ${ }^{106}$ A. A. Nepomuceno, ${ }^{23 a}$ M. Nessi, ${ }^{29, y}$ M. S. Neubauer, ${ }^{163}$ A. Neusiedl, ${ }^{79}$ R. M. Neves, ${ }^{106}$ P. Nevski, ${ }^{24}$ P. R. Newman, ${ }^{17}$ V. Nguyen Thi Hong, ${ }^{134}$ R. B. Nickerson, ${ }^{116}$ R. Nicolaidou, ${ }^{134}$ L. Nicolas, ${ }^{137}$ B. Nicquevert, ${ }^{29}$ F. Niedercorn, ${ }^{113}$ J. Nielsen, ${ }^{135}$ T. Niinikoski, ${ }^{29}$ N. Nikiforou ${ }^{34}$ A. Nikiforov ${ }^{15}$ V. Nikolaenko, ${ }^{126}$ K. Nikolaev, ${ }^{63}$ I. Nikolic-Audit, ${ }^{76}$ K. Nikolics,${ }^{48}$

K. Nikolopoulos, ${ }^{24}$ H. Nilsen, ${ }^{47}$ P. Nilsson, ${ }^{7}$ Y. Ninomiya, ${ }^{153}$ A. Nisati, ${ }^{130 a}$ T. Nishiyama, ${ }^{65}$ R. Nisius, ${ }^{97}$ L. Nodulman, ${ }^{5}$ M. Nomachi, ${ }^{114}$ I. Nomidis, ${ }^{152}$ M. Nordberg, ${ }^{29}$ B. Nordkvist, ${ }^{144 a, 144 b}$ P. R. Norton, ${ }^{127}$ J. Novakova, ${ }^{124}$ M. Nozaki, ${ }^{64}$ L. Nozka, ${ }^{111}$ I. M. Nugent, ${ }^{157 a}$ A.-E. Nuncio-Quiroz, ${ }^{20}$ G. Nunes Hanninger,${ }^{84}$ T. Nunnemann, ${ }^{96}$ E. Nurse, ${ }^{75}$ T. Nyman, ${ }^{29}$ B. J. O’Brien, ${ }^{45}$ S. W. O'Neale, ${ }^{17, a}$ D. C. O’Neil, ${ }^{140}$ V. O'Shea, ${ }^{52}$ L. B. Oakes,${ }^{96}$ F. G. Oakham, ${ }^{28, e}$ H. Oberlack, ${ }^{97}$ J. Ocariz,${ }^{76}$ A. Ochi, ${ }^{65}$ S. Oda ${ }^{153}$ S. Odaka, ${ }^{64}$ J. Odier, ${ }^{81}$ H. Ogren, ${ }^{59}$ A. Oh, ${ }^{80}$ S. H. Oh, ${ }^{44}$ C. C. Ohm, ${ }^{144 a, 144 b}$ T. Ohshima, ${ }^{99}$ H. Ohshita, ${ }^{138}$ T. Ohsugi, ${ }^{176}$ S. Okada ${ }^{65}$ H. Okawa, ${ }^{161}$ Y. Okumura, ${ }^{99}$ T. Okuyama, ${ }^{153}$ A. Olariu, ${ }^{25 a}$ M. Olcese,${ }^{49 a}$ A. G. Olchevski ${ }^{63}$ M. Oliveira, ${ }^{122 a, i}$ D. Oliveira Damazio, ${ }^{24}$ 
E. Oliver Garcia, ${ }^{165}$ D. Olivito, ${ }^{118}$ A. Olszewski, ${ }^{38}$ J. Olszowska,${ }^{38}$ C. Omachi, ${ }^{65}$ A. Onofre, ${ }^{122 a, z}$ P. U. E. Onyisi,${ }^{30}$ C. J. Oram, ${ }^{157 a}$ M. J. Oreglia, ${ }^{30}$ Y. Oren, ${ }^{151}$ D. Orestano, ${ }^{132 a, 132 b}$ I. Orlov, ${ }^{105}$ C. Oropeza Barrera, ${ }^{52}$ R. S. Orr, ${ }^{156}$ B. Osculati, ${ }^{49 a, 49 b}$ R. Ospanov, ${ }^{118}$ C. Osuna,${ }^{11}$ G. Otero y Garzon, ${ }^{26}$ J. P. Ottersbach, ${ }^{103}$ M. Ouchrif, ${ }^{133 d}$ E. A. Ouellette, ${ }^{167}$ F. Ould-Saada, ${ }^{115}$ A. Ouraou, ${ }^{134}$ Q. Ouyang, ${ }^{32 a}$ A. Ovcharova, ${ }^{14}$ M. Owen, ${ }^{80}$ S. Owen, ${ }^{137}$ V.E. Ozcan, ${ }^{18 \mathrm{a}}$ N. Ozturk, ${ }^{7}$ A. Pacheco Pages,${ }^{11}$ C. Padilla Aranda,${ }^{11}$ S. Pagan Griso, ${ }^{14}$ E. Paganis,${ }^{137}$ F. Paige,${ }^{24}$ P. Pais, ${ }^{82}$ K. Pajchel, ${ }^{115}$ G. Palacino, ${ }^{157 b}$ C. P. Paleari, ${ }^{6}$ S. Palestini, ${ }^{29}$ D. Pallin, ${ }^{33}$ A. Palma, ${ }^{122 a}$ J. D. Palmer, ${ }^{17}$ Y. B. Pan, ${ }^{170}$ E. Panagiotopoulou, ${ }^{9}$ B. Panes, ${ }^{31 a}$ N. Panikashvili, ${ }^{85}$ S. Panitkin,${ }^{24}$ D. Pantea, ${ }^{25 a}$ M. Panuskova, ${ }^{123}$ V. Paolone, ${ }^{121}$ A. Papadelis, ${ }^{144 a}$ Th. D. Papadopoulou, ${ }^{9}$ A. Paramonov, ${ }^{5}$ W. Park,${ }^{24, \text { aa }}$ M. A. Parker, ${ }^{27}$ F. Parodi, ${ }^{49 a}, 49 b$ J. A. Parsons, ${ }^{34}$ U. Parzefall, ${ }^{47}$ E. Pasqualucci, ${ }^{130 a}$ S. Passaggio, ${ }^{49 a}$ A. Passeri, ${ }^{132 a}$ F. Pastore, ${ }^{132 a, 132 b}$ Fr. Pastore, ${ }^{74}$ G. Pásztor, ${ }^{48, b b}$ S. Pataraia, ${ }^{172}$ N. Patel, ${ }^{148}$ J. R. Pater, ${ }^{80}$ S. Patricelli, ${ }^{100 a, 100 b}$ T. Pauly, ${ }^{29}$ M. Pecsy, ${ }^{142 a}$

M. I. Pedraza Morales, ${ }^{170}$ S. V. Peleganchuk, ${ }^{105}$ H. Peng, ${ }^{32 b}$ R. Pengo, ${ }^{29}$ A. Penson, ${ }^{34}$ J. Penwell, ${ }^{59}$ M. Perantoni, ${ }^{23 a}$ K. Perez,${ }^{34, c c}$ T. Perez Cavalcanti, ${ }^{41}$ E. Perez Codina, ${ }^{11}$ M. T. Pérez García-Estañ,${ }^{165}$ V. Perez Reale, ${ }^{34}$ L. Perini, ${ }^{87 \mathrm{a}, 87 \mathrm{~b}}$ H. Pernegger, ${ }^{29}$ R. Perrino, ${ }^{70 a}$ P. Perrodo,${ }^{4}$ S. Persembe, ${ }^{3 a}$ A. Perus,${ }^{113}$ V. D. Peshekhonov, ${ }^{63}$ K. Peters, ${ }^{29}$ B. A. Petersen, ${ }^{29}$ J. Petersen, ${ }^{29}$ T. C. Petersen, ${ }^{35}$ E. Petit,${ }^{4}$ A. Petridis,${ }^{152}$ C. Petridou, ${ }^{152}$ E. Petrolo, ${ }^{130 a}$ F. Petrucci, ${ }^{132 a, 132 b}$ D. Petschull, ${ }^{41}$ M. Petteni, ${ }^{140}$ R. Pezoa,${ }^{31 b}$ A. Phan,${ }^{84}$ P. W. Phillips, ${ }^{127}$ G. Piacquadio, ${ }^{29}$ E. Piccaro, ${ }^{73}$ M. Piccinini, ${ }^{19 a, 19 b}$ S. M. Piec, ${ }^{41}$ R. Piegaia, ${ }^{26}$ D. T. Pignotti, ${ }^{107}$ J. E. Pilcher, ${ }^{30}$ A. D. Pilkington, ${ }^{80}$ J. Pina, ${ }^{122 a, c}$ M. Pinamonti, ${ }^{162 a, 162 c}$ A. Pinder, ${ }^{116}$ J. L. Pinfold, ${ }^{2}$ J. Ping, ${ }^{32 c}$ B. Pinto, ${ }^{122 a, c}$ O. Pirotte, ${ }^{29}$ C. Pizio, ${ }^{87 a, 87 b}$ M. Plamondon, ${ }^{167}$ M.-A. Pleier, ${ }^{24}$ A. V. Pleskach, ${ }^{126}$ A. Poblaguev, ${ }^{24}$ S. Poddar, ${ }^{57 a}$ F. Podlyski, ${ }^{33}$ L. Poggioli, ${ }^{113}$ T. Poghosyan, ${ }^{20}$ M. Pohl,${ }^{48}$ F. Polci,${ }^{54}$ G. Polesello, ${ }^{117 a}$ A. Policicchio, ${ }^{36 a, 36 b}$ A. Polini,${ }^{19 a}$ J. Poll, ${ }^{73}$

V. Polychronakos, ${ }^{24}$ D. M. Pomarede, ${ }^{134}$ D. Pomeroy, ${ }^{22}$ K. Pommès,${ }^{29}$ L. Pontecorvo, ${ }^{130 a}$ B. G. Pope,${ }^{86}$ G. A. Popeneciu, ${ }^{25 a}$ D. S. Popovic, ${ }^{12 a}$ A. Poppleton, ${ }^{29}$ X. Portell Bueso,${ }^{29}$ C. Posch,${ }^{21}$ G. E. Pospelov, ${ }^{97}$ S. Pospisil, ${ }^{125}$ I. N. Potrap, ${ }^{97}$ C. J. Potter ${ }^{147}$ C. T. Potter,${ }^{12}$ G. Poulard, ${ }^{29}$ J. Poveda, ${ }^{170}$ R. Prabhu, ${ }^{75}$ P. Pralavorio, ${ }^{81}$ A. Pranko, ${ }^{14}$ S. Prasad, ${ }^{56}$ R. Pravahan, ${ }^{7}$ S. Prell, ${ }^{62}$ K. Pretzl,,${ }^{16}$ L. Pribyl, ${ }^{29}$ D. Price,${ }^{59}$ J. Price,${ }^{71}$ L. E. Price,${ }^{5}$ M. J. Price, ${ }^{29}$ D. Prieur, ${ }^{121}$ M. Primavera, ${ }^{70 a}$ K. Prokofiev, ${ }^{106}$ F. Prokoshin, ${ }^{31 b}$ S. Protopopescu, ${ }^{24}$ J. Proudfoot, ${ }^{5}$ X. Prudent, ${ }^{43}$ M. Przybycien, ${ }^{37}$ H. Przysiezniak, ${ }^{4}$ S. Psoroulas, ${ }^{20}$ E. Ptacek, ${ }^{112}$ E. Pueschel,${ }^{82}$ J. Purdham,${ }^{85}$ M. Purohit,${ }^{24, \text { aa }}$ P. Puzo, ${ }^{113}$ Y. Pylypchenko, ${ }^{61}$ J. Qian, ${ }^{85}$ Z. Qian, ${ }^{81}$ Z. Qin,${ }^{41}$ A. Quadt, ${ }^{53}$ D. R. Quarrie, ${ }^{14}$ W. B. Quayle, ${ }^{170}$ F. Quinonez, ${ }^{31 \mathrm{a}}$ M. Raas, ${ }^{102}$ V. Radescu, ${ }^{57 \mathrm{~b}}$ B. Radics, ${ }^{20}$ P. Radloff, ${ }^{112}$ T. Rador, ${ }^{18 \mathrm{a}}$ F. Ragusa,${ }^{87 \mathrm{a}, 87 \mathrm{~b}}$ G. Rahal, ${ }^{175}$ A. M. Rahimi, ${ }^{107}$ D. Rahm, ${ }^{24}$ S. Rajagopalan, ${ }^{24}$ M. Rammensee, ${ }^{47}$ M. Rammes, ${ }^{139}$ A. S. Randle-Conde, ${ }^{39}$ K. Randrianarivony, ${ }^{28}$ P. N. Ratoff, ${ }^{69}$ F. Rauscher, ${ }^{96}$ M. Raymond, ${ }^{29}$ A. L. Read, ${ }^{115}$ D. M. Rebuzzi, ${ }^{117 a, 117 b}$ A. Redelbach, ${ }^{171}$ G. Redlinger, ${ }^{24}$ R. Reece, ${ }^{118}$ K. Reeves, ${ }^{40}$ A. Reichold, ${ }^{103}$ E. Reinherz-Aronis, ${ }^{151}$ A. Reinsch, ${ }^{112}$ I. Reisinger, ${ }^{42}$ D. Reljic, ${ }^{12 \mathrm{a}}$ C. Rembser, ${ }^{29}$ Z. L. Ren, ${ }^{149}$ A. Renaud, ${ }^{113}$ P. Renkel, ${ }^{39}$ M. Rescigno, ${ }^{130 a}$ S. Resconi, ${ }^{87 a}$

B. Resende, ${ }^{134}$ P. Reznicek, ${ }^{96}$ R. Rezvani, ${ }^{156}$ A. Richards, ${ }^{75}$ R. Richter, ${ }^{97}$ E. Richter-Was, ${ }^{4, d d}$ M. Ridel,${ }^{76}$ M. Rijpstra, ${ }^{103}$ M. Rijssenbeek, ${ }^{146}$ A. Rimoldi, ${ }^{117 a, 117 b}$ L. Rinaldi, ${ }^{19 a}$ R. R. Rios, ${ }^{39}$ I. Riu, ${ }^{11}$ G. Rivoltella, ${ }^{87 a, 87 b}$ F. Rizatdinova, ${ }^{110}$ E. Rizvi, ${ }^{73}$ S. H. Robertson, ${ }^{83, \mathrm{k}}$ A. Robichaud-Veronneau, ${ }^{116}$ D. Robinson, ${ }^{27}$ J. E. M. Robinson, ${ }^{75}$ M. Robinson, ${ }^{112}$ A. Robson, ${ }^{52}$ J. G. Rocha de Lima, ${ }^{104}$ C. Roda, ${ }^{120 a, 120 b}$ D. Roda Dos Santos, ${ }^{29}$ D. Rodriguez, ${ }^{160}$ A. Roe,${ }^{53}$ S. Roe, ${ }^{29}$ O. Røhne, ${ }^{115}$ V. Rojo, ${ }^{1}$ S. Rolli, ${ }^{159}$ A. Romaniouk,${ }^{94}$ M. Romano, ${ }^{19 a, 19 b}$ V. M. Romanov, ${ }^{63}$ G. Romeo, ${ }^{26}$ E. Romero Adam, ${ }^{165}$ L. Roos, ${ }^{76}$ E. Ros, ${ }^{165}$ S. Rosati, ${ }^{130 a}$ K. Rosbach,${ }^{48}$ A. Rose, ${ }^{147}$ M. Rose,${ }^{74}$ G. A. Rosenbaum, ${ }^{156}$ E. I. Rosenberg, ${ }^{62}$ P. L. Rosendahl, ${ }^{13}$ O. Rosenthal, ${ }^{139}$ L. Rosselet, ${ }^{48}$ V. Rossetti, ${ }^{11}$

E. Rossi, ${ }^{130 a, 130 b}$ L. P. Rossi, ${ }^{49 a}$ M. Rotaru, ${ }^{25 a}$ I. Roth, ${ }^{169}$ J. Rothberg, ${ }^{136}$ D. Rousseau, ${ }^{113}$ C. R. Royon, ${ }^{134}$ A. Rozanov, ${ }^{81}$ Y. Rozen, ${ }^{150}$ X. Ruan, ${ }^{13, \text { ee }}$ I. Rubinskiy, ${ }^{41}$ B. Ruckert, ${ }^{96}$ N. Ruckstuhl, ${ }^{103}$ V. I. Rud, ${ }^{95}$ C. Rudolph,${ }^{43}$ G. Rudolph ${ }^{60}$ F. Rühr, ${ }^{6}$ F. Ruggieri, ${ }^{132 a, 132 b}$ A. Ruiz-Martinez, ${ }^{62}$ V. Rumiantsev,${ }^{89, a}$ L. Rumyantsev, ${ }^{63}$ K. Runge, ${ }^{47}$ Z. Rurikova, ${ }^{47}$ N. A. Rusakovich, ${ }^{63}$ D. R. Rust, ${ }^{59}$ J. P. Rutherfoord, ${ }^{6}$ C. Ruwiedel,,${ }^{14}$ P. Ruzicka, ${ }^{123}$ Y. F. Ryabov, ${ }^{119}$ V. Ryadovikov, ${ }^{126}$ P. Ryan, ${ }^{86}$ M. Rybar, ${ }^{124}$ G. Rybkin,,${ }^{13}$ N. C. Ryder, ${ }^{116}$ S. Rzaeva, ${ }^{10}$ A. F. Saavedra, ${ }^{148}$ I. Sadeh,${ }^{151}$ H. F-W. Sadrozinski, ${ }^{135}$ R. Sadykov, ${ }^{63}$ F. Safai Tehrani, ${ }^{130 a}$ H. Sakamoto, ${ }^{153}$ G. Salamanna, ${ }^{73}$ A. Salamon, ${ }^{131 a}$ M. Saleem, ${ }^{109}$ D. Salihagic, ${ }^{97}$ A. Salnikov, ${ }^{141}$ J. Salt, ${ }^{165}$ B. M. Salvachua Ferrando, ${ }^{5}$ D. Salvatore, ${ }^{36 a, 36 b}$ F. Salvatore, ${ }^{147}$ A. Salvucci, ${ }^{102}$ A. Salzburger, ${ }^{29}$ D. Sampsonidis, ${ }^{152}$ B. H. Samset, ${ }^{115}$ A. Sanchez, ${ }^{100 a, 100 b}$ H. Sandaker, ${ }^{13}$ H. G. Sander, ${ }^{79}$ M. P. Sanders,${ }^{96}$ M. Sandhoff, ${ }^{172}$ T. Sandoval, ${ }^{27}$ C. Sandoval, ${ }^{160}$ R. Sandstroem, ${ }^{97}$ S. Sandvoss, ${ }^{172}$ D. P. C. Sankey, ${ }^{127}$ A. Sansoni, ${ }^{46}$ C. Santamarina Rios, ${ }^{83}$ C. Santoni, ${ }^{33}$ R. Santonico, ${ }^{131 a, 131 b}$ H. Santos, ${ }^{122 a}$ J. G. Saraiva, ${ }^{122 a}$ T. Sarangi, ${ }^{170}$ E. Sarkisyan-Grinbaum, ${ }^{7}$ F. Sarri, ${ }^{120 a, 120 b}$ G. Sartisohn, ${ }^{172}$ O. Sasaki, ${ }^{64}$ N. Sasao, ${ }^{66}$ I. Satsounkevitch, ${ }^{88}$ G. Sauvage, ${ }^{4}$ E. Sauvan, ${ }^{4}$ J. B. Sauvan, ${ }^{113}$ P. Savard,${ }^{156, e}$ V. Savinov, ${ }^{121}$ D. O. Savu, ${ }^{29}$ L. Sawyer, ${ }^{24 \mathrm{~m}}$ D. H. Saxon, ${ }^{52}$ L. P. Says,${ }^{33}$ C. Sbarra, ${ }^{19 \mathrm{a}}$ A. Sbrizzi, ${ }^{19 \mathrm{a}, 19 \mathrm{~b}}$ O. Scallon, ${ }^{91}$ D. A. Scannicchio, ${ }^{161}$ 
M. Scarcella, ${ }^{148}$ J. Schaarschmidt, ${ }^{113}$ P. Schacht,${ }^{97}$ U. Schäfer, ${ }^{79}$ S. Schaepe, ${ }^{20}$ S. Schaetzel,${ }^{57 b}$ A. C. Schaffer, ${ }^{113}$ D. Schaile, ${ }^{96}$ R. D. Schamberger, ${ }^{146}$ A. G. Schamov, ${ }^{105}$ V. Scharf, ${ }^{57 a}$ V. A. Schegelsky, ${ }^{119}$ D. Scheirich,${ }^{85}$ M. Schernau, ${ }^{161}$ M. I. Scherzer, ${ }^{34}$ C. Schiavi, ${ }^{49 a, 49 b}$ J. Schieck,${ }^{96}$ M. Schioppa, ${ }^{36 a, 36 b}$ S. Schlenker, ${ }^{29}$ J. L. Schlereth, ${ }^{5}$ E. Schmidt ${ }^{47}$ K. Schmieden, ${ }^{20}$ C. Schmitt,,${ }^{79}$ S. Schmitt, ${ }^{57 b}$ M. Schmitz, ${ }^{20}$ A. Schöning, ${ }^{57 b}$ M. Schott, ${ }^{29}$ D. Schouten, ${ }^{157 \mathrm{a}}$ J. Schovancova, ${ }^{123}$ M. Schram, ${ }^{83}$ C. Schroeder, ${ }^{79}$ N. Schroer, ${ }^{57 \mathrm{c}}$ S. Schuh, ${ }^{29}$ G. Schuler, ${ }^{29}$ J. Schultes, ${ }^{172}$ H.-C. Schultz-Coulon, ${ }^{57 a}$ H. Schulz, ${ }^{15}$ J. W. Schumacher, ${ }^{20}$ M. Schumacher, ${ }^{47}$ B. A. Schumm, ${ }^{135}$ Ph. Schune, ${ }^{134}$ C. Schwanenberger, ${ }^{80}$ A. Schwartzman, ${ }^{141}$ Ph. Schwemling, ${ }^{76}$ R. Schwienhorst, ${ }^{86}$ R. Schwierz, ${ }^{43}$ J. Schwindling, ${ }^{134}$ T. Schwindt, ${ }^{20}$ M. Schwoerer, ${ }^{4}$ W. G. Scott, ${ }^{127}$ J. Searcy,${ }^{12}$ G. Sedov, ${ }^{41}$ E. Sedykh, ${ }^{119}$ E. Segura, ${ }^{11}$ S. C. Seidel, ${ }^{101}$ A. Seiden, ${ }^{135}$ F. Seifert, ${ }^{43}$ J. M. Seixas, ${ }^{23 a}$ G. Sekhniaidze, ${ }^{100 a}$ K. E. Selbach, ${ }^{45}$ D. M. Seliverstov,${ }^{119}$ B. Sellden, ${ }^{144 a}$ G. Sellers, ${ }^{71}$ M. Seman, ${ }^{142 b}$ N. Semprini-Cesari, ${ }^{19 a, 19 b}$ C. Serfon, ${ }^{96}$ L. Serin, ${ }^{113}$ R. Seuster, ${ }^{97}$ H. Severini, ${ }^{109}$ M. E. Sevior, ${ }^{84}$ A. Sfyrla, ${ }^{29}$ E. Shabalina,${ }^{53}$ M. Shamim, ${ }^{112}$ L. Y. Shan, ${ }^{32 a}$ J. T. Shank, ${ }^{21}$ Q. T. Shao, ${ }^{84}$ M. Shapiro, ${ }^{14}$ P. B. Shatalov, ${ }^{93}$ L. Shaver, ${ }^{6}$ K. Shaw, ${ }^{162 a, 162 \mathrm{c}}$ D. Sherman, ${ }^{173}$ P. Sherwood,${ }^{75}$ A. Shibata, ${ }^{106}$ H. Shichi, ${ }^{99}$ S. Shimizu, ${ }^{29}$ M. Shimojima, ${ }^{98}$ T. Shin, ${ }^{55}$ M. Shiyakova, ${ }^{63}$ A. Shmeleva,${ }^{92}$ M. J. Shochet,${ }^{30}$ D. Short, ${ }^{116}$ S. Shrestha, ${ }^{62}$ M. A. Shupe ${ }^{6}$ P. Sicho, ${ }^{123}$ A. Sidoti ${ }^{130 a}$ F. Siegert ${ }^{47}$ Dj. Sijacki, ${ }^{12 a}$ O. Silbert, ${ }^{169}$ J. Silva, ${ }^{122 a, c}$ Y. Silver, ${ }^{151}$ D. Silverstein, ${ }^{141}$ S. B. Silverstein, ${ }^{144 a}$ V. Simak, ${ }^{125}$ O. Simard, ${ }^{134}$ Lj. Simic,${ }^{12 a}$ S. Simion, ${ }^{113}$ B. Simmons,${ }^{75}$ M. Simonyan, ${ }^{35}$ P. Sinervo, ${ }^{156}$ N. B. Sinev, ${ }^{112}$ V. Sipica, ${ }^{139}$ G. Siragusa, ${ }^{171}$ A. Sircar, ${ }^{24}$ A. N. Sisakyan, ${ }^{63}$ S. Yu. Sivoklokov, ${ }^{95}$ J. Sjölin, ${ }^{144 a, 144 b}$ T. B. Sjursen, ${ }^{13}$ L. A. Skinnari, ${ }^{14}$ H. P. Skottowe, ${ }^{56}$ K. Skovpen, ${ }^{105}$ P. Skubic, ${ }^{109}$ N. Skvorodnev, ${ }^{22}$ M. Slater, ${ }^{17}$ T. Slavicek, ${ }^{125}$ K. Sliwa, ${ }^{159}$ J. Sloper,${ }^{29}$ V. Smakhtin, ${ }^{169}$ S. Yu. Smirnov, ${ }^{94}$ L. N. Smirnova, ${ }^{95}$ O. Smirnova, ${ }^{77}$ B. C. Smith,${ }^{56}$ D. Smith, ${ }^{141}$ K. M. Smith, ${ }^{52}$ M. Smizanska, ${ }^{69}$ K. Smolek, ${ }^{125}$ A. A. Snesarev, ${ }^{92}$ S. W. Snow, ${ }^{80}$ J. Snow, ${ }^{109}$ J. Snuverink, ${ }^{103}$ S. Snyder, ${ }^{24}$ M. Soares, ${ }^{122 a}$ R. Sobie, ${ }^{167, k}$

J. Sodomka, ${ }^{125}$ A. Soffer ${ }^{151}$ C. A. Solans, ${ }^{165}$ M. Solar, ${ }^{125}$ J. Solc,${ }^{125}$ E. Soldatov,${ }^{94}$ U. Soldevila, ${ }^{165}$

E. Solfaroli Camillocci, ${ }^{130 a, 130 b}$ A. A. Solodkov, ${ }^{126}$ O. V. Solovyanov, ${ }^{126}$ N. Soni, ${ }^{2}$ V. Sopko, ${ }^{125}$ B. Sopko, ${ }^{125}$ M. Sosebee, ${ }^{7}$ R. Soualah, ${ }^{162 a, 162 c}$ A. Soukharev ${ }^{105}$ S. Spagnolo, ${ }^{70 a, 70 b}$ F. Spanò,${ }^{74}$ R. Spighi, ${ }^{19 a}$ G. Spigo, ${ }^{29}$ F. Spila, ${ }^{130 a, 130 b}$ R. Spiwoks, ${ }^{29}$ M. Spousta, ${ }^{124}$ T. Spreitzer, ${ }^{156}$ B. Spurlock, ${ }^{7}$ R. D. St. Denis, ${ }^{52}$ T. Stahl,,${ }^{139}$ J. Stahlman, ${ }^{118}$ R. Stamen, ${ }^{57 a}$ E. Stanecka, ${ }^{38}$ R. W. Stanek, ${ }^{5}$ C. Stanescu, ${ }^{132 a}$ S. Stapnes, ${ }^{115}$ E. A. Starchenko, ${ }^{126}$ J. Stark, ${ }^{54}$ P. Staroba, ${ }^{123}$ P. Starovoitov, ${ }^{89}$ A. Staude, ${ }^{96}$ P. Stavina, ${ }^{142 a}$ G. Stavropoulos, ${ }^{14}$ G. Steele,${ }^{52}$ P. Steinbach, ${ }^{43}$ P. Steinberg, ${ }^{24}$ I. Stekl, ${ }^{125}$ B. Stelzer, ${ }^{140}$ H. J. Stelzer, ${ }^{86}$ O. Stelzer-Chilton, ${ }^{157 a}$ H. Stenzel, ${ }^{51}$ S. Stern, ${ }^{97}$

K. Stevenson, ${ }^{73}$ G. A. Stewart, ${ }^{29}$ J. A. Stillings, ${ }^{20}$ M. C. Stockton, ${ }^{83}$ K. Stoerig, ${ }^{47}$ G. Stoicea, ${ }^{25 a}$ S. Stonjek, ${ }^{97}$ P. Strachota, ${ }^{124}$ A. R. Stradling, ${ }^{7}$ A. Straessner, ${ }^{43}$ J. Strandberg, ${ }^{145}$ S. Strandberg, ${ }^{14 a, 144 b}$ A. Strandlie, ${ }^{115}$ M. Strang, ${ }^{107}$ E. Strauss, ${ }^{141}$ M. Strauss ${ }^{109}$ P. Strizenec, ${ }^{142 b}$ R. Ströhmer, ${ }^{171}$ D. M. Strom, ${ }^{112}$ J. A. Strong, ${ }^{74, a}$ R. Stroynowski, ${ }^{39}$ J. Strube, ${ }^{127}$ B. Stugu, ${ }^{13}$ I. Stumer, ${ }^{24, a}$ J. Stupak, ${ }^{146}$ P. Sturm, ${ }^{172}$ N. A. Styles, ${ }^{41}$ D. A. Soh, ${ }^{149, v}$

D. Su, ${ }^{141}$ HS. Subramania, ${ }^{2}$ A. Succurro, ${ }^{11}$ Y. Sugaya, ${ }^{114}$ T. Sugimoto, ${ }^{99}$ C. Suhr,${ }^{104}$ K. Suita, ${ }^{65}$ M. Suk,${ }^{124}$ V. V. Sulin, ${ }^{92}$ S. Sultansoy, ${ }^{3 d}$ T. Sumida,${ }^{66}$ X. Sun,${ }^{54}$ J. E. Sundermann,${ }^{47}$ K. Suruliz, ${ }^{137}$ S. Sushkov, ${ }^{11}$ G. Susinno, ${ }^{36 a, 36 b}$ M. R. Sutton, ${ }^{147}$ Y. Suzuki, ${ }^{64}$ Y. Suzuki, ${ }^{65}$ M. Svatos, ${ }^{123}$ Yu. M. Sviridov, ${ }^{126}$ S. Swedish, ${ }^{166}$ I. Sykora, ${ }^{142 a}$ T. Sykora, ${ }^{124}$ B. Szeless,${ }^{29}$ J. Sánchez, ${ }^{165} \mathrm{D}$. Ta, ${ }^{103}$ K. Tackmann, ${ }^{41}$ A. Taffard,${ }^{161}$ R. Tafirout, ${ }^{157 a}$ N. Taiblum, ${ }^{151}$ Y. Takahashi,${ }^{99}$ H. Takai, ${ }^{24}$ R. Takashima, ${ }^{67}$ H. Takeda, ${ }^{65}$ T. Takeshita, ${ }^{138}$ Y. Takubo, ${ }^{64}$ M. Talby, ${ }^{81}$ A. Talyshev, ${ }^{105,9}$ M. C. Tamsett, ${ }^{24}$ J. Tanaka,${ }^{153}$ R. Tanaka, ${ }^{113}$ S. Tanaka, ${ }^{129}$ S. Tanaka, ${ }^{64}$ Y. Tanaka, ${ }^{98}$ A. J. Tanasijczuk, ${ }^{140}$ K. Tani ${ }^{65}$ N. Tannoury, ${ }^{81}$ G. P. Tappern, ${ }^{29}$ S. Tapprogge, ${ }^{79}$ D. Tardif,${ }^{156}$ S. Tarem, ${ }^{150}$ F. Tarrade,${ }^{28}$ G. F. Tartarelli, ${ }^{87 a}$ P. Tas, ${ }^{124}$ M. Tasevsky, ${ }^{123}$ E. Tassi,${ }^{36 a, 36 b}$ M. Tatarkhanov, ${ }^{14}$ Y. Tayalati, ${ }^{133 d}$ C. Taylor ${ }^{75}$ F. E. Taylor, ${ }^{90}$ G. N. Taylor, ${ }^{84}$ W. Taylor, ${ }^{157 b}$ M. Teinturier, ${ }^{113}$ M. Teixeira Dias Castanheira, ${ }^{73}$ P. Teixeira-Dias, ${ }^{74}$ K. K. Temming,${ }^{47}$ H. Ten Kate,${ }^{29}$ P. K. Teng, ${ }^{149}$ S. Terada,${ }^{64}$ K. Terashi,${ }^{153}$ J. Terron, ${ }^{78}$ M. Testa, ${ }^{46}$ R. J. Teuscher, ${ }^{156, \mathrm{k}}$ J. Thadome, ${ }^{172} \mathrm{~J}$. Therhaag, ${ }^{20} \mathrm{~T}$. Theveneaux-Pelzer,${ }^{76} \mathrm{M}$. Thioye, ${ }^{173} \mathrm{~S}$. Thoma ${ }^{47} \mathrm{~J} . \mathrm{P} . \mathrm{Thomas},{ }^{17}$ E. N. Thompson, ${ }^{34}$ P. D. Thompson, ${ }^{17}$ P. D. Thompson, ${ }^{156}$ A. S. Thompson, ${ }^{52}$ E. Thomson, ${ }^{118}$ M. Thomson, ${ }^{27}$ R. P. Thun, ${ }^{85}$ F. Tian, ${ }^{34}$ M. J. Tibbetts, ${ }^{14}$ T. Tic,${ }^{123}$ V. O. Tikhomirov, ${ }^{92}$ Y. A. Tikhonov, ${ }^{105,9}$ S. Timoshenko, ${ }^{94}$ P. Tipton, ${ }^{173}$ F. J. Tique Aires Viegas, ${ }^{29}$ S. Tisserant,,${ }^{81}$ B. Toczek,,${ }^{37}$ T. Todorov, ${ }^{4}$ S. Todorova-Nova, ${ }^{159}$

B. Toggerson, ${ }^{161}$ J. Tojo,${ }^{64} \mathrm{~S}$. Tokár, ${ }^{142 \mathrm{a}} \mathrm{K}$. Tokunaga,${ }^{65} \mathrm{~K}$. Tokushuku, ${ }^{64} \mathrm{~K}$. Tollefson, ${ }^{86} \mathrm{M}$. Tomoto, ${ }^{99}$ L. Tompkins,${ }^{30} \mathrm{~K}$. Toms, ${ }^{101}$ G. Tong, ${ }^{32 a}$ A. Tonoyan, ${ }^{13}$ C. Topfel,${ }^{16}$ N. D. Topilin, ${ }^{63}$ I. Torchiani, ${ }^{29}$ E. Torrence, ${ }^{112}$ H. Torres, ${ }^{76}$ E. Torró Pastor, ${ }^{165}$ J. Toth, ${ }^{81, b b}$ F. Touchard, ${ }^{81}$ D. R. Tovey ${ }^{137}$ T. Trefzger, ${ }^{171}$ L. Tremblet, ${ }^{29}$ A. Tricoli, ${ }^{29}$ I. M. Trigger ${ }^{157 a}$ S. Trincaz-Duvoid, ${ }^{76}$ T. N. Trinh, ${ }^{76}$ M. F. Tripiana ${ }^{68} \mathrm{~W}$. Trischuk,${ }^{156}$ A. Trivedi, ${ }^{24, \text { aa }}$ B. Trocmé, ${ }^{54}$ C. Troncon, ${ }^{87 \mathrm{a}} \mathrm{M}$. Trottier-McDonald, ${ }^{140} \mathrm{M}$. Trzebinski, ${ }^{38}$ A. Trzupek, ${ }^{38}$ C. Tsarouchas, ${ }^{29}$ J. C-L. Tseng, ${ }^{116}$ M. Tsiakiris, ${ }^{103}$ P. V. Tsiareshka, ${ }^{88}$ D. Tsionou, ${ }^{4, f f}$ G. Tsipolitis, ${ }^{9}$ V. Tsiskaridze,${ }^{47}$ E. G. Tskhadadze,${ }^{50 a}$ 
I. I. Tsukerman, ${ }^{93}$ V. Tsulaia, ${ }^{14}$ J.-W. Tsung, ${ }^{20}$ S. Tsuno, ${ }^{64}$ D. Tsybychev, ${ }^{146}$ A. Tua, ${ }^{137}$ A. Tudorache,${ }^{25 a}$

V. Tudorache, ${ }^{25 \mathrm{a}}$ J. M. Tuggle, ${ }^{30} \mathrm{M}$. Turala, ${ }^{38} \mathrm{D}$. Turecek, ${ }^{125}$ I. Turk Cakir, ${ }^{3 \mathrm{e}} \mathrm{E}$. Turlay, ${ }^{103}$ R. Turra, ${ }^{87 a, 87 \mathrm{~b}}$ P. M. Tuts, ${ }^{34}$ A. Tykhonov, ${ }^{72}$ M. Tylmad,${ }^{144 a, 144 b}$ M. Tyndel,${ }^{127}$ G. Tzanakos, ${ }^{8}$ K. Uchida,${ }^{20}$ I. Ueda, ${ }^{153}$ R. Ueno, ${ }^{28}$ M. Ugland, ${ }^{13}$ M. Uhlenbrock, ${ }^{20}$ M. Uhrmacher, ${ }^{53}$ F. Ukegawa, ${ }^{158}$ G. Unal, ${ }^{29}$ D. G. Underwood, ${ }^{5}$ A. Undrus,${ }^{24}$ G. Unel, ${ }^{161}$ Y. Unno, ${ }^{64}$ D. Urbaniec,${ }^{34}$ G. Usai, ${ }^{7}$ M. Uslenghi, ${ }^{117 a, 117 b}$ L. Vacavant, ${ }^{81}$ V. Vacek, ${ }^{125}$ B. Vachon, ${ }^{83}$

S. Vahsen, ${ }^{14}$ J. Valenta, ${ }^{123}$ P. Valente, ${ }^{130 a}$ S. Valentinetti, ${ }^{19 a, 19 b}$ S. Valkar, ${ }^{124}$ E. Valladolid Gallego, ${ }^{165}$ S. Vallecorsa, ${ }^{150}$ J. A. Valls Ferrer, ${ }^{165}$ H. van der Graaf, ${ }^{103}$ E. van der Kraaij, ${ }^{103}$ R. Van Der Leeuw, ${ }^{103}$ E. van der Poel, ${ }^{103}$ D. van der Ster, ${ }^{29}$ N. van Eldik, ${ }^{82}$ P. van Gemmeren, ${ }^{5}$ Z. van Kesteren, ${ }^{103}$ I. van Vulpen, ${ }^{103}$ M. Vanadia, ${ }^{97}$ W. Vandelli, ${ }^{29}$ G. Vandoni ${ }^{29}$ A. Vaniachine, ${ }^{5}$ P. Vankov, ${ }^{41}$ F. Vannucci, ${ }^{76}$ F. Varela Rodriguez, ${ }^{29}$ R. Vari, ${ }^{130 a}$ E. W. Varnes, ${ }^{6}$ D. Varouchas,${ }^{14}$ A. Vartapetian, ${ }^{7}$ K. E. Varvell,${ }^{148}$ V. I. Vassilakopoulos,${ }^{55}$ F. Vazeille, ${ }^{33}$ G. Vegni, ${ }^{87 a, 87 b}$ J. J. Veillet,${ }^{113}$ C. Vellidis, ${ }^{8}$ F. Veloso,${ }^{122 a}$ R. Veness, ${ }^{29}$ S. Veneziano, ${ }^{130 a}$ A. Ventura, ${ }^{70 a, 70 b}$ D. Ventura, ${ }^{136} \mathrm{M}$. Venturi, ${ }^{47} \mathrm{~N}$. Venturi, ${ }^{156} \mathrm{~V}$. Vercesi, ${ }^{117 a} \mathrm{M}$. Verducci, ${ }^{136} \mathrm{~W}$. Verkerke, ${ }^{103} \mathrm{~J}$. C. Vermeulen, ${ }^{103}$ A. Vest, ${ }^{43}$ M. C. Vetterli, ${ }^{140, \mathrm{e}}$ I. Vichou, ${ }^{163}$ T. Vickey, ${ }^{143 \mathrm{~b}, \mathrm{gg}}$ O.E. Vickey Boeriu, ${ }^{143 \mathrm{~b}}$ G. H. A. Viehhauser, ${ }^{116}$ S. Viel, ${ }^{166}$ M. Villa, ${ }^{19 a, 19 b}$ M. Villaplana Perez, ${ }^{165}$ E. Vilucchi, ${ }^{46}$ M. G. Vincter ${ }^{28}$ E. Vinek,${ }^{29}$ V. B. Vinogradov, ${ }^{63}$ M. Virchaux,${ }^{134, \text { a }}$ J. Virzi,${ }^{14}$ O. Vitells, ${ }^{169}$ M. Viti ${ }^{41}$ I. Vivarelli, ${ }^{47}$ F. Vives Vaque,${ }^{2}$ S. Vlachos, ${ }^{9}$ D. Vladoiu, ${ }^{96}$ M. Vlasak, ${ }^{125}$ N. Vlasov,${ }^{20}$ A. Vogel,${ }^{20}$ P. Vokac, ${ }^{125}$ G. Volpi ${ }^{46}$ M. Volpi,${ }^{84}$ G. Volpini,${ }^{87 a}$ H. von der Schmitt, ${ }^{97}$ J. von Loeben, ${ }^{97}$ H. von Radziewski, ${ }^{47}$ E. von Toerne, ${ }^{20}$ V. Vorobel, ${ }^{124}$ A. P. Vorobiev, ${ }^{126}$ V. Vorwerk, ${ }^{11}$ M. Vos, ${ }^{165}$ R. Voss, ${ }^{29}$ T. T. Voss, ${ }^{172}$ J.H. Vossebeld, ${ }^{71}$ N. Vranjes, ${ }^{12 a}$ M. Vranjes Milosavljevic, ${ }^{103}$ V. Vrba, ${ }^{123}$ M. Vreeswijk, ${ }^{103}$

T. Vu Anh, ${ }^{79}$ R. Vuillermet, ${ }^{29}$ I. Vukotic, ${ }^{113}$ W. Wagner, ${ }^{172}$ P. Wagner, ${ }^{18}$ H. Wahlen, ${ }^{172}$ J. Wakabayashi, ${ }^{99}$ J. Walbersloh, ${ }^{42}$ S. Walch,${ }^{85}$ J. Walder,${ }^{69}$ R. Walker, ${ }^{96}$ W. Walkowiak, ${ }^{139}$ R. Wall, ${ }^{173}$ P. Waller, ${ }^{71} \mathrm{C}$. Wang, ${ }^{44}$ H. Wang, ${ }^{170}$ H. Wang, ${ }^{32 b, h h}$ J. Wang, ${ }^{149}$ J. Wang, ${ }^{54}$ J.C. Wang, ${ }^{136}$ R. Wang, ${ }^{101}$ S. M. Wang, ${ }^{149}$ A. Warburton, ${ }^{83}$

C. P. Ward,${ }^{27}$ M. Warsinsky, ${ }^{47}$ P. M. Watkins, ${ }^{17}$ A. T. Watson, ${ }^{17}$ I. J. Watson,,${ }^{148}$ M. F. Watson, ${ }^{17}$ G. Watts, ${ }^{136}$ S. Watts, ${ }^{80}$ A. T. Waugh, ${ }^{148}$ B. M. Waugh,${ }^{75}$ M. Weber, ${ }^{127}$ M. S. Weber, ${ }^{16}$ P. Weber, ${ }^{53}$ A. R. Weidberg,,${ }^{16}$ P. Weigell, ${ }^{97}$ J. Weingarten, ${ }^{53}$ C. Weiser,${ }^{47}$ H. Wellenstein, ${ }^{22}$ P. S. Wells,${ }^{29}$ M. Wen, ${ }^{46}$ T. Wenaus, ${ }^{24}$ S. Wendler, ${ }^{121}$ Z. Weng, ${ }^{149, v}$

T. Wengler, ${ }^{29}$ S. Wenig, ${ }^{29}$ N. Wermes,${ }^{20}$ M. Werner, ${ }^{47}$ P. Werner, ${ }^{29}$ M. Werth,${ }^{161}$ M. Wessels, ${ }^{57 a} \mathrm{C}$. Weydert, ${ }^{54}$ K. Whalen, ${ }^{28}$ S. J. Wheeler-Ellis, ${ }^{161}$ S. P. Whitaker ${ }^{21}$ A. White, ${ }^{7}$ M. J. White,${ }^{84}$ S. R. Whitehead, ${ }^{116}$ D. Whiteson, ${ }^{161}$ D. Whittington, ${ }^{59}$ F. Wicek, ${ }^{113}$ D. Wicke, ${ }^{172}$ F. J. Wickens, ${ }^{127}$ W. Wiedenmann, ${ }^{170}$ M. Wielers, ${ }^{127}$ P. Wienemann, ${ }^{20}$ C. Wiglesworth, ${ }^{73}$ L. A. M. Wiik-Fuchs, ${ }^{47}$ P. A. Wijeratne, ${ }^{75}$ A. Wildauer, ${ }^{165}$ M. A. Wildt,${ }^{41, r}$ I. Wilhelm, ${ }^{124}$ H. G. Wilkens, ${ }^{29}$ J. Z. Will,${ }^{96}$ E. Williams,${ }^{34}$ H. H. Williams, ${ }^{118}$ W. Willis, ${ }^{34}$ S. Willocq, ${ }^{82}$ J. A. Wilson, ${ }^{17}$ M. G. Wilson, ${ }^{141}$ A. Wilson, ${ }^{85}$ I. Wingerter-Seez,${ }^{4}$ S. Winkelmann, ${ }^{47}$ F. Winklmeier, ${ }^{29}$ M. Wittgen, ${ }^{141}$ M. W. Wolter, ${ }^{38}$ H. Wolters, ${ }^{122 a, i}$ W. C. Wong, ${ }^{40}$ G. Wooden, ${ }^{85}$ B. K. Wosiek, ${ }^{38}$ J. Wotschack, ${ }^{29}$ M. J. Woudstra, ${ }^{82}$ K. W. Wozniak, ${ }^{38}$ K. Wraight, ${ }^{52}$ C. Wright, ${ }^{52}$ M. Wright,${ }^{52}$ B. Wrona,${ }^{71}$ S. L. Wu, ${ }^{170}$ X. Wu, ${ }^{48}$ Y. Wu, ${ }^{32 b, i i}$ E. Wulf, ${ }^{34}$

R. Wunstorf, ${ }^{42}$ B. M. Wynne, ${ }^{45}$ S. Xella, ${ }^{35}$ M. Xiao, ${ }^{134}$ S. Xie, ${ }^{47}$ Y. Xie, ${ }^{32 a}$ C. Xu, ${ }^{32 b, x}$ D. Xu, ${ }^{137}$ G. Xu, ${ }^{32 a}$

B. Yabsley, ${ }^{148}$ S. Yacoob, ${ }^{143 b}$ M. Yamada, ${ }^{64}$ H. Yamaguchi, ${ }^{153}$ A. Yamamoto,${ }^{64}$ K. Yamamoto, ${ }^{62}$ S. Yamamoto, ${ }^{153}$ T. Yamamura, ${ }^{153}$ T. Yamanaka, ${ }^{153}$ J. Yamaoka, ${ }^{44}$ T. Yamazaki, ${ }^{153}$ Y. Yamazaki, ${ }^{65}$ Z. Yan, ${ }^{21}$ H. Yang, ${ }^{85}$ U. K. Yang,${ }^{80}$ Y. Yang, ${ }^{59}$ Y. Yang, ${ }^{32 a}$ Z. Yang, ${ }^{144 a, 144 b}$ S. Yanush,${ }^{89}$ Y. Yao, ${ }^{14}$ Y. Yasu,${ }^{64}$ G. V. Ybeles Smit,${ }^{128}$ J. Ye, ${ }^{39}$ S. Ye ${ }^{24}$ M. Yilmaz, ${ }^{3 \mathrm{c}}$ R. Yoosoofmiya, ${ }^{121}$ K. Yorita, ${ }^{168}$ R. Yoshida, ${ }^{5}$ C. Young, ${ }^{141}$ S. Youssef,${ }^{21}$ D. Yu, ${ }^{24}$ J. Yu, ${ }^{7}$ J. Yu, ${ }^{110}$ L. Yuan, ${ }^{32 a, j j}$ A. Yurkewicz, ${ }^{104}$ B. Zabinski, ${ }^{38}$ V. G. Zaets, ${ }^{126}$ R. Zaidan, ${ }^{61}$ A. M. Zaitsev, ${ }^{126}$ Z. Zajacova, ${ }^{29}$ L. Zanello, ${ }^{130 a, 130 b}$ P. Zarzhitsky, ${ }^{39}$ A. Zaytsev, ${ }^{105}$ C. Zeitnitz, ${ }^{172}$ M. Zeller, ${ }^{173}$ M. Zeman, ${ }^{123}$ A. Zemla ${ }^{38}$ C. Zendler, ${ }^{20}$ O. Zenin, ${ }^{126}$ T. Ženišs, ${ }^{142 a}$ Z. Zinonos, ${ }^{120 a, 120 b}$ S. Zenz, ${ }^{14}$ D. Zerwas, ${ }^{113}$ G. Zevi della Porta, ${ }^{56}$ Z. Zhan, ${ }^{32 \mathrm{~d}}$ D. Zhang, ${ }^{32 \mathrm{~b}, \mathrm{hh}}$ H. Zhang, ${ }^{86}$ J. Zhang, ${ }^{5}$ X. Zhang, ${ }^{32 \mathrm{~d}}$ Z. Zhang, ${ }^{113} \mathrm{~L}$. Zhao, ${ }^{106}$ T. Zhao, ${ }^{136}$ Z. Zhao, ${ }^{32 \mathrm{~b}}$ A. Zhemchugov, ${ }^{63}$ S. Zheng, ${ }^{32 \mathrm{a}}$ J. Zhong, ${ }^{116}$ B. Zhou, ${ }^{85}$ N. Zhou, ${ }^{161}$ Y. Zhou, ${ }^{149}$ C. G. Zhu, ${ }^{32 \mathrm{~d}}$ H. Zhu, ${ }^{41}$ J. Zhu, ${ }^{85}$ Y. Zhu, ${ }^{32 \mathrm{~b}}$ X. Zhuang, ${ }^{96}$ V. Zhuravlov ${ }^{97}$ D. Zieminska, ${ }^{59}$ R. Zimmermann, ${ }^{20}$ S. Zimmermann, ${ }^{20}$ S. Zimmermann, ${ }^{47}$ M. Ziolkowski, ${ }^{139}$ R. Zitoun, ${ }^{4}$ L. Živković, ${ }^{34}$ V. V. Zmouchko, ${ }^{126, a}$ G. Zobernig, ${ }^{170}$ A. Zoccoli, ${ }^{19 a, 19 b}$ Y. Zolnierowski, ${ }^{4}$ A. Zsenei, ${ }^{29}$ M. zur Nedden, ${ }^{15}$ V. Zutshi, ${ }^{104}$ and L. Zwalinski ${ }^{29}$

(ATLAS Collaboration)

\author{
${ }^{1}$ University at Albany, Albany, New York, USA \\ ${ }^{2}$ Department of Physics, University of Alberta, Edmonton, Alberta, Canada \\ ${ }^{3 a}$ Department of Physics, Ankara University, Ankara, Turkey \\ ${ }^{3 \mathrm{~b}}$ Department of Physics, Dumlupinar University, Kutahya, Turkey
}


${ }^{3 \mathrm{c}}$ Department of Physics, Gazi University, Ankara, Turkey

${ }^{3 \mathrm{~d}}$ Division of Physics, TOBB University of Economics and Technology, Ankara, Turkey

${ }^{3 \mathrm{e}}$ Turkish Atomic Energy Authority, Ankara, Turkey

${ }^{4} L A P P, C N R S / I N 2 P 3$ and Université de Savoie, Annecy-le-Vieux, France

${ }^{5}$ High Energy Physics Division, Argonne National Laboratory, Argonne, Illinois, USA

${ }^{6}$ Department of Physics, University of Arizona, Tucson, Arizona, USA

${ }^{7}$ Department of Physics, The University of Texas at Arlington, Arlington Texas, USA

${ }^{8}$ Physics Department, University of Athens, Athens, Greece

${ }^{9}$ Physics Department, National Technical University of Athens, Zografou, Greece

${ }^{10}$ Institute of Physics, Azerbaijan Academy of Sciences, Baku, Azerbaijan

${ }^{11}$ Institut de Física d'Altes Energies and Departament de Física de la Universitat Autònoma de Barcelona and ICREA, Barcelona, Spain

${ }^{12 \mathrm{a}}$ Institute of Physics, University of Belgrade, Belgrade, Serbia

${ }^{12 \mathrm{~b}}$ Vinca Institute of Nuclear Sciences, University of Belgrade, Belgrade, Serbia

${ }^{13}$ Department for Physics and Technology, University of Bergen, Bergen, Norway

${ }^{14}$ Physics Division, Lawrence Berkeley National Laboratory and University of California, Berkeley, California, USA

${ }^{15}$ Department of Physics, Humboldt University, Berlin, Germany

${ }^{16}$ Albert Einstein Center for Fundamental Physics and Laboratory for High Energy Physics, University of Bern, Bern, Switzerland

${ }^{17}$ School of Physics and Astronomy, University of Birmingham, Birmingham, United Kingdom

${ }^{18 a}$ Department of Physics, Bogazici University, Istanbul, Turkey

${ }^{18 \mathrm{~b}}$ Division of Physics, Dogus University, Istanbul, Turkey

${ }^{18 \mathrm{c}}$ Department of Physics Engineering, Gaziantep University, Gaziantep, Turkey

${ }^{18 \mathrm{~d} D e p a r t m e n t}$ of Physics, Istanbul Technical University, Istanbul, Turkey

${ }^{19 a}$ INFN Sezione di Bologna, Bologna, Italy

${ }^{19 \mathrm{~b}}$ Dipartimento di Fisica, Università di Bologna, Bologna, Italy

${ }^{20}$ Physikalisches Institut, University of Bonn, Bonn, Germany

${ }^{21}$ Department of Physics, Boston University, Boston, Massachusetts, USA

${ }^{22}$ Department of Physics, Brandeis University, Waltham, Massachusetts, USA

${ }^{23 a}$ Universidade Federal do Rio De Janeiro COPPE/EE/IF, Rio de Janeiro, Brazil

${ }^{23 \mathrm{~b}}$ Federal University of Juiz de Fora (UFJF), Juiz de Fora, Brazil

${ }^{23 \mathrm{c}}$ Federal University of Sao Joao del Rei (UFSJ), Sao Joao del Rei, Brazil

${ }^{23 \mathrm{~d}}$ Instituto de Fisica, Universidade de Sao Paulo, Sao Paulo, Brazil

${ }^{24}$ Physics Department, Brookhaven National Laboratory, Upton, New York, USA

${ }^{25 a}$ National Institute of Physics and Nuclear Engineering, Bucharest, Romania

${ }^{25 \mathrm{~b}}$ University Politehnica Bucharest, Bucharest, Romania

${ }^{25 \mathrm{c}}$ West University in Timisoara, Timisoara, Romania

${ }^{26}$ Departamento de Física, Universidad de Buenos Aires, Buenos Aires, Argentina

${ }^{27}$ Cavendish Laboratory, University of Cambridge, Cambridge, United Kingdom

${ }^{28}$ Department of Physics, Carleton University, Ottawa, Ontario, Canada ${ }^{29}$ CERN, Geneva, Switzerland

${ }^{30}$ Enrico Fermi Institute, University of Chicago, Chicago, Illinois, USA

${ }^{31 a}$ Departamento de Fisica, Pontificia Universidad Católica de Chile, Santiago, Chile

${ }^{31 \mathrm{~b}}$ Departamento de Física, Universidad Técnica Federico Santa María, Valparaíso, Chile

${ }^{32 \mathrm{a}}$ Institute of High Energy Physics, Chinese Academy of Sciences, Beijing, China

${ }^{32 \mathrm{~b}}$ Department of Modern Physics, University of Science and Technology of China, Anhui, China

${ }^{32 \mathrm{c}}$ Department of Physics, Nanjing University, Jiangsu, China

${ }^{32 \mathrm{~d}}$ School of Physics, Shandong University, Shandong, China

${ }^{33}$ Laboratoire de Physique Corpusculaire, Clermont Université

and Université Blaise Pascal and CNRS/IN2P3, Aubiere Cedex, France

${ }^{34}$ Nevis Laboratory, Columbia University, Irvington, New York, USA

${ }^{35}$ Niels Bohr Institute, University of Copenhagen, Kobenhavn, Denmark

${ }^{36 a}$ INFN Gruppo Collegato di Cosenza, Italy

${ }^{36 \mathrm{~b}}$ Dipartimento di Fisica, Università della Calabria, Arcavata di Rende, Italy

${ }^{37}$ AGH University of Science and Technology, Faculty of Physics and Applied Computer Science, Krakow, Poland

${ }^{38}$ The Henryk Niewodniczanski Institute of Nuclear Physics, Polish Academy of Sciences, Krakow, Poland

${ }^{39}$ Physics Department, Southern Methodist University, Dallas, Texas, USA

${ }^{40}$ Physics Department, University of Texas at Dallas, Richardson, Texas, USA

${ }^{41}$ DESY, Hamburg and Zeuthen, Germany

${ }^{42}$ Institut für Experimentelle Physik IV, Technische Universität Dortmund, Dortmund, Germany

${ }^{43}$ Institut für Kern- und Teilchenphysik, Technical University Dresden, Dresden, Germany

${ }^{44}$ Department of Physics, Duke University, Durham, North Carolina, USA 
${ }^{45}$ SUPA-School of Physics and Astronomy, University of Edinburgh, Edinburgh, United Kingdom

${ }^{46}$ INFN Laboratori Nazionali di Frascati, Frascati, Italy

${ }^{47}$ Fakultät für Mathematik und Physik, Albert-Ludwigs-Universität, Freiburg i.Br., Germany

${ }^{48}$ Section de Physique, Université de Genève, Geneva, Switzerland

${ }^{49 a}$ INFN Sezione di Genova, Genova, Italy

${ }^{49 \mathrm{~b}}$ Dipartimento di Fisica, Università di Genova, Genova, Italy

${ }^{50 \mathrm{a}}$ E. Andronikashvili Institute of Physics, Tbilisi State University, Tbilisi, Georgia

${ }^{50 \mathrm{~b}}$ High Energy Physics Institute, Tbilisi State University, Tbilisi, Georgia

${ }^{51}$ II Physikalisches Institut, Justus-Liebig-Universität Giessen, Giessen, Germany

${ }^{52}$ SUPA_School of Physics and Astronomy, University of Glasgow, Glasgow, United Kingdom

${ }^{53}$ II Physikalisches Institut, Georg-August-Universität, Göttingen, Germany

${ }^{54}$ Laboratoire de Physique Subatomique et de Cosmologie, Université Joseph Fourier and CNRS/IN2P3 and Institut National Polytechnique de Grenoble, Grenoble, France

${ }^{55}$ Department of Physics, Hampton University, Hampton, Virginia, USA

${ }^{56}$ Laboratory for Particle Physics and Cosmology, Harvard University, Cambridge, Massachusetts, USA

${ }^{57}$ Kirchhoff-Institut für Physik, Ruprecht-Karls-Universität Heidelberg, Heidelberg, Germany

${ }^{57 b}$ Physikalisches Institut, Ruprecht-Karls-Universität Heidelberg, Heidelberg, Germany

${ }^{57 \mathrm{c}}$ ZITI Institut für technische Informatik, Ruprecht-Karls-Universität Heidelberg, Mannheim, Germany

${ }^{58}$ Faculty of Applied Information Science, Hiroshima Institute of Technology, Hiroshima, Japan

${ }^{59}$ Department of Physics, Indiana University, Bloomington, Indiana, USA

${ }^{60}$ Institut für Astro- und Teilchenphysik, Leopold-Franzens-Universität, Innsbruck, Austria

${ }^{61}$ University of Iowa, Iowa City, Iowa, USA

${ }^{62}$ Department of Physics and Astronomy, Iowa State University, Ames, Iowa, USA

${ }^{63}$ Joint Institute for Nuclear Research, JINR Dubna, Dubna, Russia

${ }^{64}$ KEK, High Energy Accelerator Research Organization, Tsukuba, Japan

${ }^{65}$ Graduate School of Science, Kobe University, Kobe, Japan

${ }^{66}$ Faculty of Science, Kyoto University, Kyoto, Japan

${ }^{67}$ Kyoto University of Education, Kyoto, Japan

${ }^{68}$ Instituto de Física La Plata, Universidad Nacional de La Plata and CONICET, La Plata, Argentina

${ }^{69}$ Physics Department, Lancaster University, Lancaster, United Kingdom

${ }^{70 \mathrm{a}}$ INFN Sezione di Lecce, Lecce, Italy

${ }^{70 \mathrm{~b}}$ Dipartimento di Fisica, Università del Salento, Lecce, Italy

${ }^{71}$ Oliver Lodge Laboratory, University of Liverpool, Liverpool, United Kingdom

${ }^{72}$ Department of Physics, Jožef Stefan Institute and University of Ljubljana, Ljubljana, Slovenia

${ }^{73}$ School of Physics and Astronomy, Queen Mary University of London, London, United Kingdom

${ }^{74}$ Department of Physics, Royal Holloway University of London, Surrey, United Kingdom

${ }^{75}$ Department of Physics and Astronomy, University College London, London, United Kingdom

${ }^{76}$ Laboratoire de Physique Nucléaire et de Hautes Energies, UPMC and Université Paris-Diderot and CNRS/IN2P3, Paris France

${ }^{77}$ Fysiska institutionen, Lunds universitet, Lund, Sweden

${ }^{78}$ Departamento de Fisica Teorica C-15, Universidad Autonoma de Madrid, Madrid, Spain

${ }^{79}$ Institut für Physik, Universität Mainz, Mainz, Germany

${ }^{80}$ School of Physics and Astronomy, University of Manchester, Manchester, United Kingdom

${ }^{81}$ CPPM, Aix-Marseille Université and CNRS/IN2P3, Marseille, France

${ }^{82}$ Department of Physics, University of Massachusetts, Amherst, Massachusetts, USA

${ }^{83}$ Department of Physics, McGill University, Montreal, Quebec, Canada

${ }^{84}$ School of Physics, University of Melbourne, Victoria, Australia

${ }^{85}$ Department of Physics, The University of Michigan, Ann Arbor Michigan, United States of America, USA

${ }^{86}$ Department of Physics and Astronomy, Michigan State University, East Lansing, Michigan, USA

${ }^{87 a}$ INFN Sezione di Milano, Milano, Italy

${ }^{87 \mathrm{~b}}$ Dipartimento di Fisica, Università di Milano, Milano, Italy

${ }^{88}$ B.I. Stepanov Institute of Physics, National Academy of Sciences of Belarus, Minsk, Republic of Belarus

${ }^{89}$ National Scientific and Educational Centre for Particle and High Energy Physics, Minsk, Republic of Belarus

${ }^{90}$ Department of Physics, Massachusetts Institute of Technology, Cambridge, Massachusetts, USA

${ }^{91}$ Group of Particle Physics, University of Montreal, Montreal, Quebec, Canada

${ }^{92}$ P.N. Lebedev Institute of Physics, Academy of Sciences, Moscow, Russia

${ }^{93}$ Institute for Theoretical and Experimental Physics (ITEP), Moscow, Russia

${ }^{94}$ Moscow Engineering and Physics Institute (MEPhI), Moscow, Russia

${ }^{95}$ Skobeltsyn Institute of Nuclear Physics, Lomonosov Moscow State University, Moscow, Russia

${ }^{96}$ Fakultät für Physik, Ludwig-Maximilians-Universität München, München, Germany

${ }^{97}$ Max-Planck-Institut für Physik (Werner-Heisenberg-Institut), München, Germany

${ }^{98}$ Nagasaki Institute of Applied Science, Nagasaki, Japan 
${ }^{99}$ Graduate School of Science, Nagoya University, Nagoya, Japan

${ }^{100 a}$ INFN Sezione di Napoli, Napoli, Italy

${ }^{100 \mathrm{~b}}$ Dipartimento di Scienze Fisiche, Università di Napoli, Napoli, Italy

${ }^{101}$ Department of Physics and Astronomy, University of New Mexico, Albuquerque, New Mexico, USA

${ }^{102}$ Institute for Mathematics, Astrophysics and Particle Physics, Radboud University Nijmegen/Nikhef, Nijmegen, Netherlands

${ }^{103}$ Nikhef National Institute for Subatomic Physics and University of Amsterdam, Amsterdam, Netherlands

${ }^{104}$ Department of Physics, Northern Illinois University, DeKalb, Illinois, USA

${ }^{105}$ Budker Institute of Nuclear Physics, SB RAS, Novosibirsk, Russia

${ }^{106}$ Department of Physics, New York University, New York, New York, USA

${ }^{107}$ Ohio State University, Columbus, Ohio, USA

${ }^{108}$ Faculty of Science, Okayama University, Okayama, Japan

${ }^{109}$ Homer L. Dodge Department of Physics and Astronomy, University of Oklahoma, Norman, Oklahoma, USA

${ }^{110}$ Department of Physics, Oklahoma State University, Stillwater Oklahoma, USA

${ }^{111}$ Palacky University, RCPTM, Olomouc, Czech Republic

${ }^{112}$ Center for High Energy Physics, University of Oregon, Eugene Oregon, USA

${ }^{113}$ LAL, Univ. Paris-Sud and CNRS/IN2P3, Orsay, France

${ }^{114}$ Graduate School of Science, Osaka University, Osaka, Japan

${ }^{115}$ Department of Physics, University of Oslo, Oslo, Norway

${ }^{116}$ Department of Physics, Oxford University, Oxford, United Kingdom

${ }^{117 a}$ INFN Sezione di Pavia, Pavia, Italy

${ }^{117 b}$ Dipartimento di Fisica, Università di Pavia, Pavia, Italy

${ }^{118}$ Department of Physics, University of Pennsylvania, Philadelphia Pennsylvania, USA

${ }^{119}$ Petersburg Nuclear Physics Institute, Gatchina, Russia

${ }^{120 a}$ INFN Sezione di Pisa, Pisa, Italy

${ }^{120 b}$ Dipartimento di Fisica E. Fermi, Università di Pisa, Pisa, Italy

${ }^{121}$ Department of Physics and Astronomy, University of Pittsburgh, Pittsburgh, Pennsylvania, USA

${ }^{122 a}$ Laboratorio de Instrumentacao e Fisica Experimental de Particulas-LIP, Lisboa, Portugal

${ }^{122 b}$ Departamento de Fisica Teorica y del Cosmos and CAFPE, Universidad de Granada, Granada, Spain

${ }^{123}$ Institute of Physics, Academy of Sciences of the Czech Republic, Praha, Czech Republic

${ }^{124}$ Faculty of Mathematics and Physics, Charles University in Prague, Praha, Czech Republic

${ }^{125}$ Czech Technical University in Prague, Praha, Czech Republic

${ }^{126}$ State Research Center Institute for High Energy Physics, Protvino, Russia

${ }^{127}$ Particle Physics Department, Rutherford Appleton Laboratory, Didcot, United Kingdom

${ }^{128}$ Physics Department, University of Regina, Regina, Saskatchewan, Canada

${ }^{129}$ Ritsumeikan University, Kusatsu, Shiga, Japan

${ }^{130 a}$ INFN Sezione di Roma I, Roma, Italy

${ }^{130 \mathrm{~b}}$ Dipartimento di Fisica, Università La Sapienza, Roma, Italy

${ }^{131 \mathrm{a}}$ INFN Sezione di Roma Tor Vergata, Roma, Italy

${ }^{131 \mathrm{~b}}$ Dipartimento di Fisica, Università di Roma Tor Vergata, Roma, Italy

${ }^{132 a}$ INFN Sezione di Roma Tre, Roma, Italy

${ }^{132 b}$ Dipartimento di Fisica, Università Roma Tre, Roma, Italy

${ }^{133 a}$ Faculté des Sciences Ain Chock, Réseau Universitaire de Physique des Hautes

Energies-Université Hassan II, Casablanca, Morocco

${ }^{133 b}$ Centre National de l'Energie des Sciences Techniques Nucleaires, Rabat, Morocco

${ }^{133 c}$ Faculté des Sciences Semlalia, Université Cadi Ayyad, LPHEA-Marrakech, Morocco

${ }^{133 \mathrm{~d}}$ Faculté des Sciences, Université Mohamed Premier and LPTPM, Oujda, Morocco

${ }^{133 e}$ Faculté des Sciences, Université Mohammed V-Agdal, Rabat, Morocco

${ }^{134}$ DSM/IRFU (Institut de Recherches sur les Lois Fondamentales de l'Univers),

CEA Saclay (Commissariat a l'Energie Atomique), Gif-sur-Yvette, France

${ }^{135}$ Santa Cruz Institute for Particle Physics, University of California Santa Cruz, Santa Cruz, California, USA

${ }^{136}$ Department of Physics, University of Washington, Seattle Washington, USA

${ }^{137}$ Department of Physics and Astronomy, University of Sheffield, Sheffield, United Kingdom

${ }^{138}$ Department of Physics, Shinshu University, Nagano, Japan

${ }^{139}$ Fachbereich Physik, Universität Siegen, Siegen, Germany

${ }^{140}$ Department of Physics, Simon Fraser University, Burnaby, British Columbia, Canada

${ }^{141}$ SLAC National Accelerator Laboratory, Stanford, California, USA

${ }^{142 a}$ Faculty of Mathematics, Physics \& Informatics, Comenius University, Bratislava, Slovak Republic

${ }^{142 \mathrm{~b}}$ Department of Subnuclear Physics, Institute of Experimental Physics of the Slovak Academy of Sciences, Kosice, Slovak Republic

${ }^{143 a}$ Department of Physics, University of Johannesburg, Johannesburg, South Africa

${ }^{143 \mathrm{~b}}$ School of Physics, University of the Witwatersrand, Johannesburg, South Africa

${ }^{144 a}$ Department of Physics, Stockholm University, Sweden 


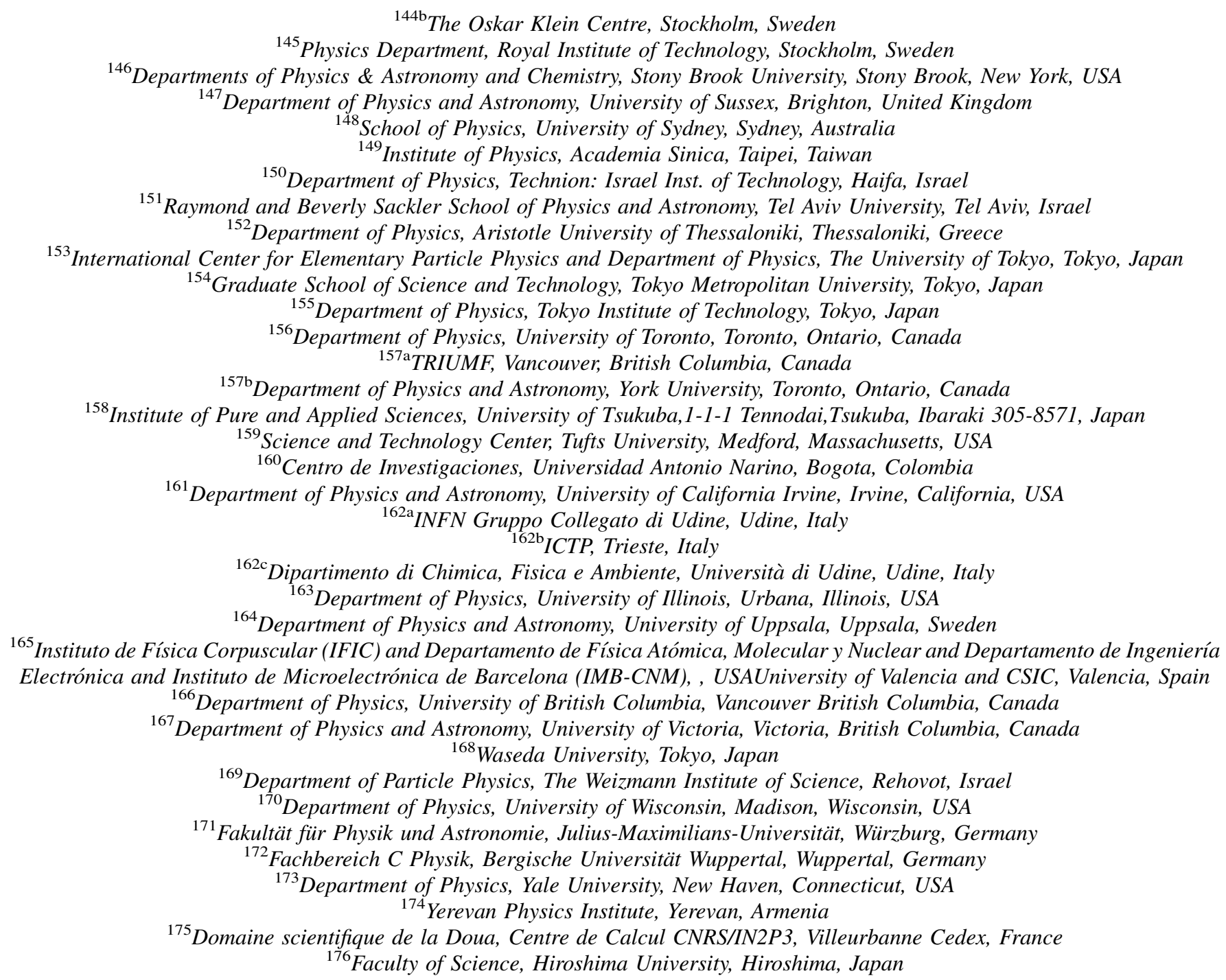

${ }^{\mathrm{a}}$ Deceased.

${ }^{\mathrm{b}}$ Also at Laboratorio de Instrumentacao e Fisica Experimental de Particulas-LIP, Lisboa, Portugal

${ }^{\mathrm{c}}$ Also at Faculdade de Ciencias and CFNUL, Universidade de Lisboa, Lisboa, Portugal

${ }^{\mathrm{d}}$ Also at Particle Physics Department, Rutherford Appleton Laboratory, Didcot, United Kingdom

${ }^{\mathrm{e}}$ Also at TRIUMF, Vancouver BC, Canada

${ }^{\mathrm{f}}$ Also at Department of Physics, California State University, Fresno, CA, USA

${ }^{\mathrm{g}}$ Also at Novosibirsk State University, Novosibirsk, Russia

${ }^{\mathrm{h}}$ Also at Fermilab, Batavia, IL, USA

${ }^{\mathrm{i}}$ Also at Department of Physics, Coimbra, Portugal

${ }^{\mathrm{j}}$ Also at Università di Napoli Parthenope, Napoli, Italy

${ }^{\mathrm{k}}$ Also at Institute of Particle Physics (IPP), Canada

${ }^{1}$ Also at Department of Physics, Middle East Technical University, Ankara, Turkey

${ }^{\mathrm{m}}$ Also at Louisiana Tech University, Ruston, LA, USA

${ }^{\mathrm{n}}$ Also at Department of Physics and Astronomy, University College London, London, United Kingdom

${ }^{\circ}$ Also at Group of Particle Physics, University of Montreal, Montreal, QC, Canada

${ }^{\mathrm{p}}$ Also at Department of Physics, University of Cape Town, Cape Town, South Africa

${ }^{\mathrm{q}}$ Also at Institute of Physics, Azerbaijan Academy of Sciences, Baku, Azerbaijan

${ }^{\mathrm{r}}$ Also at Institut für Experimentalphysik, Universität Hamburg, Hamburg, Germany

${ }^{\mathrm{s}}$ Also at Manhattan College, New York, NY, USA

${ }^{\mathrm{t}}$ Also at School of Physics, Shandong University, Shandong, China

${ }^{u}$ Also at CPPM, Aix-Marseille Université and CNRS/IN2P3, Marseille, France 
${ }^{v}$ Also at School of Physics and Engineering, Sun Yat-sen University, Guanzhou, China

${ }^{w}$ Also at Academia Sinica Grid Computing, Institute of Physics, Academia Sinica, Taipei, Taiwan

${ }^{x}$ Also at DSM/IRFU (Institut de Recherches sur les Lois Fondamentales de l'Univers), CEA Saclay (Commissariat a l'Energie Atomique), Gif-sur-Yvette, France

${ }^{y}$ Also at Section de Physique, Université de Genève, Geneva, Switzerland

${ }^{\mathrm{z}}$ Also at Departamento de Fisica, Universidade de Minho, Braga, Portugal

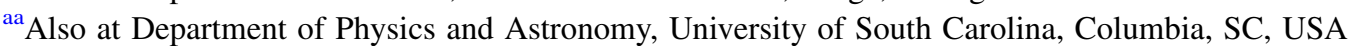

${ }^{\mathrm{bb}} \mathrm{Also}$ at Institute for Particle and Nuclear Physics, Wigner Research Centre for Physics, Budapest, Hungary

${ }^{\mathrm{cc}}$ Also at California Institute of Technology, Pasadena, CA, USA

${ }^{\mathrm{dd}}$ Also at Institute of Physics, Jagiellonian University, Krakow, Poland

${ }^{e e}$ Also at Institute of High Energy Physics, Chinese Academy of Sciences, Beijing, China

${ }^{\mathrm{ff}}$ Also at Department of Physics and Astronomy, University of Sheffield, Sheffield, United Kingdom

${ }^{g g}$ Also at Department of Physics, Oxford University, Oxford, United Kingdom

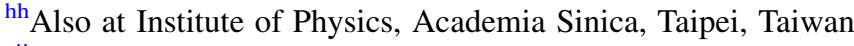

${ }^{i i}$ Also at Department of Physics, The University of Michigan, Ann Arbor, MI, USA

${ }^{\mathrm{jj}}$ Also at Laboratoire de Physique Nucléaire et de Hautes Energies, UPMC and Université Paris-Diderot and CNRS/IN2P3, Paris, France 